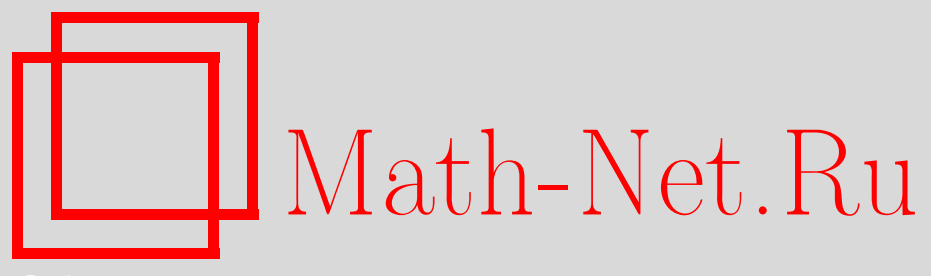

А. Л. Кери, Ф. А. Сукочев, Следы Диксмье и некоторые приложения в некоммутативной геометрии, УМН, 2006, том 61, выпуск 6, 45-110

DOI: https://doi.org/10.4213/rm4262

Использование Общероссийского математического портала Math-Net.Ru подразумевает, что вы прочитали и согласны с пользовательским соглашением http://www . mathnet.ru/rus/agreement

Параметры загрузки:

IP : 18.209 .158 .208

26 апреля 2023 г., 15:06:53

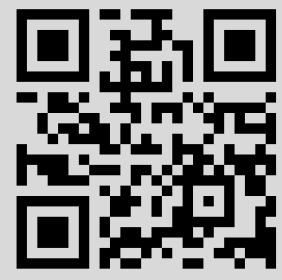




\title{
Следы Диксмье и некоторые приложения в некоммутативной геометрии
}

\author{
А. Л. Кери, Ф. А. Сукочев
}

Мы обсуждаем недавние продвижения в теории сингулярных следов на идеалах компактных операторов, концентрируясь на следах Диксмье и их приложениях в некоммутативной геометрии. Нашим отправным пунктом является книга Алена Конна "Noncommutative geometry", содержащая различные открытые проблемы и мотивировки для их изучения. Одна из особенностей нашего изложения состоит в том, что мы работаем с операторными идеалами в общих полуконечных алгебрах фон Неймана. Хотя многие из описываемых нами результатов уже встречались в литературе, в некоторых случаях мы вводим новые или улучшенные доказательства. Мы отсылаем читателя к оглавлению по поводу сводки тем, затронутых в данной работе.

Библиография: 95 названий.

СОДЕРЖАНИЕ

1. Введение............................................. 46

2. Предварительные сведения: пространства и функционалы .......... 50

2.1. Пространства Марцинкевича функций и последовательностей . . 50

2.2. Сингулярные симметричные функционалы на пространствах Мар-

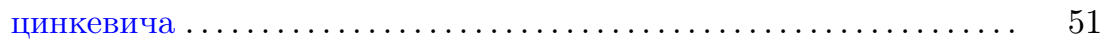

2.3. Симметричные операторные пространства и функционалы .... 52

3. Общие факты о симметричных функционалах................. 54

4. Предварительные сведения о состояниях, инвариантных при растяже-

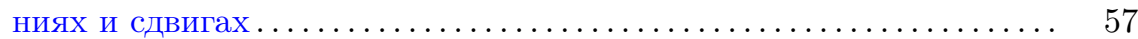

5. Конкретные конструкции сингулярных симметричных функционалов . 61

5.1. Следы Диксмье ................................. 61

5.2. Следы Конна-Диксмье............................... 64

5.3. Перестановочно-инвариантные функционалы и сингулярные сле-

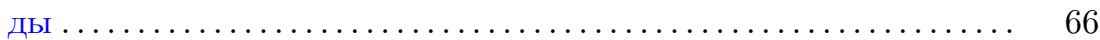

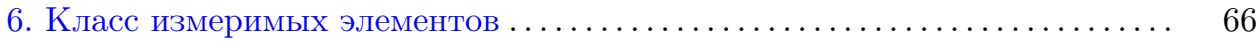

7. Нормирующие свойства функционалов Диксмье и Конна-Диксмье .... 69

(C) А. Л. Кери, Ф.А. Сукочев, 2006 
8. Фредгольмовы модули и спектральные тройки ................... 71

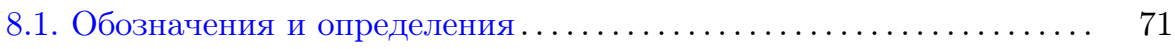

8.2. Сопоставление ограниченных и неограниченных модулей ...... 72

8.3. Дополнительная информация о полуконечных спектральных трой-

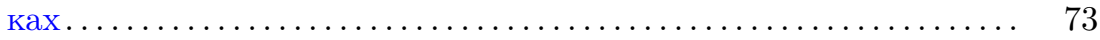

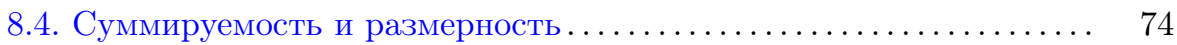

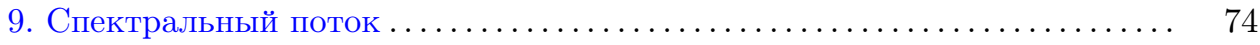

9.1. Формулы для спектрального потока.................. 76

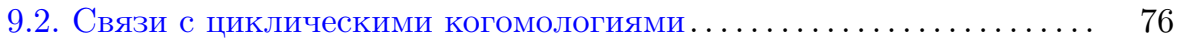

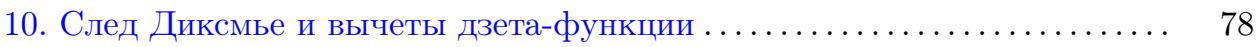

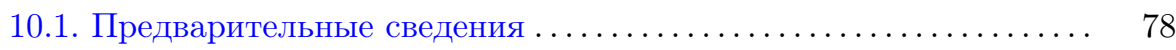

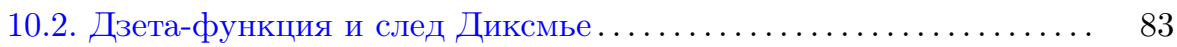

11. Формула в терминах уравнения теплопроводности ............. 87

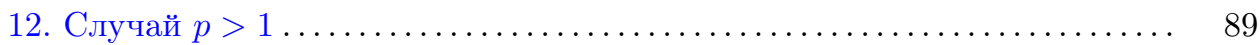

13. Обобщенные тёплицевы операторы и их индекс ................ 90

14. Негладкие слоения и псевдодифференциальные операторы . . . . . . . 92

15. Алгебра почти периодических функций $\ldots \ldots \ldots \ldots \ldots \ldots \ldots \ldots \ldots . . \ldots \ldots$

15.1. Почти периодические псевдодифференциальные операторы . . 94

15.2. Почти периодическая спектральная тройка ............. 98

16. Теорема Леша об индексе .............................. 99

17. Класс Хохшильда характера Черна........................... 102

18. Формула типа Лидского для следов Диксмье ..................... 104

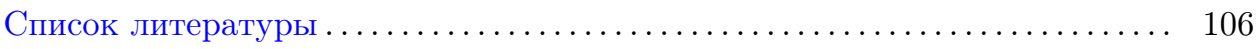

\section{1. Введение}

След Диксмье $\tau_{\omega}$ возник из задачи о том, имеет ли алгебра $B(\mathscr{H})$ всех ограниченных линейных операторов на гильбертовом пространстве $\mathscr{H}$ единственный нетривиальный след. Ж. Диксмье разрешил этот вопрос отрицательно в 1966 г. в заметке, опубликованной в журнале "Comptes Rendus" [1]. Для того чтобы построить след, он использовал инвариантное среднее $\omega$ на разрешимой “ $a x+b "$-группе. Введенный им след обращается в нуль на идеале ядерных операторов и, следовательно, полностью отличен от обычного следа. Он, к тому же, не является нормальным.

Приложения следа Диксмье в классической геометрии облегчаются замечательным результатом [2], связывающим этот след с вычетом Водзицкого [3] для псевдодифференциальных операторов на замкнутом многообразии. Последний был введен независимо М. Адлером [4], Ю. И. Маниным [5] в одномерном случае и далее развит М. Водзицким [3] и В. Гийемином [6] в высших размерностях. Интригующее и самое полезное свойство вычета Водзицкого, не считая его вычислимости в примерах, заключается в том факте, что он имеет смысл для псевдодифференциальных операторов произвольного порядка. Более того, он является единственным следом на псевдодифференциальных операторах, который продолжает след Диксмье на операторах порядка, не превосходящего минус размерность подлежащего многообразия [2]. 
Как след Диксмье, так и вычет Водзицкого играют важную роль в некоммутативной геометрии и ее приложениях [7], [8]. В частности, след Диксмье $\tau_{\omega}$ является подходящим некоммутативным аналогом интегрирования на компактном $n$-мерном римановом спинорном многообразии $M$, точнее,

$$
\tau_{\omega}\left(f(x)|D|^{-n}\right)=\frac{1}{(2 \pi)^{n} n} 2^{[n / 2]} \Omega_{n-1} \int_{M} f(x) d \mathrm{vol},
$$

где $f$ - гладкая функция на $M, D$ - оператор Дирака на $M, \Omega_{n-1}$ - объем $(n-1)$-мерной единичной сферы и $d \mathrm{vol}$ - риманова форма объема. Полезность следа Диксмье расширяется результатами А. Конна [7], который связал его с вычетами дзета-функций.

Имеются важные приложения следа Диксмье в теории гравитации, классической теории поля и физике частиц, которые мы не имеем возможности включить ввиду недостатка места. Они хорошо изложены в книге [8] и берут свое начало в связи между вычетом Водзицкого степеней оператора Дирака и действием Эйнштейна-Гильберта [2]. Равным образом, мы не пытаемся изложить другой материал, содержащийся в книгах [7] и [8], которые описывают несколько интересных физических приложений следа Диксмье. Мы отсылаем читателя к обзору [9] и к обширной литературе, касающейся приложений некоммутативной геометрии к "стандартной модели” физики частиц, где, начиная с элегантного выражения для принципа некоммутативного действия, выраженного в терминах следа Ж. Диксмье, А. Конн и Дж. Лотт [10] и А. Конн [11] показали, как вывести из него евклидову версию действия для стандартной модели. Аналогичным образом, можно также дать некоммутативную формулировку гамильтоновой версии классической теории поля, снова используя след Диксмье [12]. Некоторая базисная информация об этих результатах представлена в работах [13], [14].

Эти приложения в физике восходят к исследовательскому сообщению [15], в котором было описано несколько основополагающих результатов (некоторые из них позднее были собраны в книге [7]). После вычисления следа Диксмье для псевдодифференциальных операторов, Конн получил действия Янга-Миллса и Полякова, начиная с функционала действия, содержащего след Диксмье. Там также была анонсирована теорема о формуле вычетов для класса Хохшильда характера Черна, которую мы описываем в разделе 17. Следующий шаг был сделан в работе [16], в которой Конн ввел аксиомы некоммутативной спинорной геометрии для некоммутативной алгебры $\mathscr{A}$. Самая первая аксиома использует след Диксмье, чтобы ввести теорию некоммутативного интегрирования на $\mathscr{A}$, что вполне естественно ввиду формулы (1). Эта точка зрения снова всплывает на поверхность в нашем обсуждении теоремы Леша об индексе в разделе 16, хотя мы не будем далее отклоняться и вводить другие аксиомы Конна, исключая упоминание роли класса Хохшильда в разделе 17.

Хотя упомянутые выше приложения к физике служат стимулом для исследований, они не являются центральным объектом этой статьи. Наша задача состоит в том, чтобы дать цельное и логически последовательное изложение некоторых недавних функционально-аналитических продвижений в теории следов Диксмье. В дополнение к обзору этих новых результатов, в некоторых случаях мы также предлагаем новые доказательства. Частично, нашей мотивировкой 
является обобщение и прояснение вопросов, поставленных в [7; ч. IV]. Конкретнее, мы приводим характеризацию класса измеримых операторов, определенных в [7], объясняем роль средних по Чезаро в конновской версии следов Диксмье (называемых в настоящей статье следами Конна-Диксмье) и даем полный анализ формул для следа Диксмье в терминах дзета-функций. Более важным, однако, является то, что все наши рассмотрения проводятся в рамках "полуконечных спектральных троек", которые мы объясняем в разделе 8. Это понятие появляется в случае, если распространять теорию, изложенную в работе [7], имеющую дело с подалгебрами ограниченных операторов на сепарабельном гильбертовом пространстве, снабженном стандартным следом, на ситуацию, в которой алгебра фон Неймана ограниченных операторов заменяется общей полуконечной алгеброй фон Неймана, снабженной некоторым точным нормальным полуконечным следом [17]. Как результат дополнительных усилий, необходимых для того, чтобы справиться с этим уровнем общности, оказалось возможным найти усовершенствования даже в стандартной теории типа $I$, развитой в [7]. Несколько авторов внесли свой вклад в развитие этого подхода, а также новых приложений следов Диксмье и более общих сингулярных следов [18]-[39].

Наше изложение организовано вокруг трех основных вопросов. Изучение первого круга вопросов преобладает в начальной части статьи (достигая своей кульминации в разделе 6) и дает характеризацию измеримых операторов в смысле [7; раздел IV.2]. Мы подходим к данной теме с точки зрения общей теории сингулярных симметричных функционалов (этот вопрос и изложение его предварительных сведений занимают разделы 2-5). Наше изложение основано на подходе, описанном явно в работах [31], [29], который рассматривает такие следы как специальный класс непрерывных линейных функционалов на соответствующих операторных идеалах. Многие характерные особенности теории можно хорошо понять даже в самой тривиальной ситуации, когда рассматриваемые алгебры фон Неймана коммутативны. В этой ситуации теория следов Диксмье примерно соответствует теории симметричних функционалов на перестановочно-инвариантных функциональных пространствах [40], [41], [31], и потому она допускает альтернативную трактовку, основанную на привлечении методов теории функций действительного переменного.

Затем, готовясь к оставшейся части работы, мы вводим понятия полуконечной спектральной тройки, спектрального потока типа $I I$ и некоторые обозначения для циклических когомологий. Это приводит нас ко второму кругу вопросов, занимающих разделы 10 и 12, где мы описываем выражение следа Диксмье при помощи вычетов дзета-функций. Главное наблюдение, сделанное в разделе 4, которое позволяет нам доказать значительное обобщение предложения IV.2.4 из книги [7], а также теорему об измеримости из раздела 6, заключается в существовании двух видов следа Диксмье. Один из них ассоциирован с мультипликативной группой положительных действительных чисел и ее инвариантным средним, а второй, который естественно ассоциируется с дзета-функцией, возникает из инвариантного среднего аддитивной группы вещественных чисел. Связь между этими двумя видами дает формулу для следа Диксмье в терминах дзета-функции.

Эти результаты используют язык спектральных троек и естественно приводят к третьему кругу рассматриваемых вопросов, а именно, к некоторым 
приложениям, которые начинаются в разделе 11. Мы приводим доказательство (обобщения) формулы, данной в [7; с. 563] для следа Диксмье в терминах уравнения теплопроводности. Затем мы приводим формулу для индекса обобщенных тёплицевых операторов (раздел 13), описываем специальный случай формулы вычета Водзицкого, полученной М.-Т. Бенамью и Т. Факом [22] для псевдодифференциальных операторов вдоль слоев слоения, и обсуждаем в разделе 15 подобную формулу для псевдодифференциальных операторов с почти периодическими коэффициентами. Формула раздела 13 имеет в качестве следствия теорему Леша об индексе для тёплицевых операторов с некоммутативным символом, и это описывается в разделе 16. Обобщение теоремы IV.2.8 из [7] о формуле вычетов для класса Хохшильда характера Черна фредгольмовых модулей приведено в разделе 17 (см. также [22]).

Наконец, следуя работе [21], мы показываем в разделе 18, как можно распространить формулу Лидского на следы Диксмье. В ряде следствий мы объясняем их значимость в вопросе об измеримости некоторых классических операторов. В [7], за исключением случая псевдодифференциальных операторов, результаты об измеримости доказаны для положительных операторов. Подход работы [21] позволяет обратиться к задаче устранения предположения положительности.

Ниже мы приводим краткий список символов и терминов, используемых в этой работе, с указанием места, где они вводятся:

- симметричные и перестановочно-инвариантные функционалы и перестановочно-инвариантные пространства $E(J)$ (определения $2.1,2.3$ );

- пространства Марцинкевича $M(\psi), \mathscr{L}^{(1, \infty)}, \mathscr{L}^{(p, \infty)}$ (п. 2.1);

- функция обобщенных сингулярных значений $\mu_{(\cdot)}(x)$ (п. 2.3);

- полуконечная алгебра фон Неймана $\mathscr{N}$; точный нормальный полуконечный след на $\mathscr{N}$, обозначаемый $\tau$; $\tau$-измеримые операторы $\widetilde{\mathscr{N}}$; вполне симметричное операторное пространство на $(\mathscr{N}, \tau)$, порожденное банаховым функциональным пространством $E$, обозначаемое $E(\mathscr{N}, \tau)$ (п. 2.3);

- операторные пространства Марцинкевича $M(\psi)(\mathscr{N}, \tau)$ (п. 2.3);

- симметричные функционалы на вполне симметричном пространстве $E=$ $E(0, \infty): E_{\text {sym }}^{*}($ п. 3$)$;

- множества состояний $B L(\mathbb{R}), D\left(\mathbb{R}_{+}^{*}\right), B L\left(\mathbb{R}_{+}\right)$(раздел 4);

- следы Диксмье $\tau_{\omega}$ (определения 5.1, 5.4), следы $F_{\mathbb{L}}, F_{\mathbf{L}}$ (п. 5.1);

- следы Конна-Диксмье $\tau_{\omega}$ с $\omega \in C D\left(\mathbb{R}_{+}^{*}\right)$ (п. 5.2);

- измеримые операторы (определения 6.1 и 6.2 );

- спектральные тройки $(\mathscr{A}, \mathscr{H}, \mathscr{D})$, градуирующий оператор $\Gamma, \mathscr{I}$-суммируемость (определение 8.1);

- полуконечные спектральные тройки класса $Q C^{k}$ (определение 8.6);

- спектральная размерность $(\mathscr{A}, \mathscr{H}, \mathscr{D})$ (определение 8.7);

- спектральный поток $\operatorname{sf}\left(\left\{F_{t}\right\}\right), \operatorname{sf}\left(D, u D u^{*}\right)$ (раздел 9$)$;

- $(b, B)$-коцепь, $(b, B)$-коцикл, $\left(b^{T}, B^{T}\right)$-цепь, $\left(b^{T}, B^{T}\right)$-цикл (раздел 9$)$;

- дзета-функции $\zeta(s), \zeta_{A}(s)$ (п. 10.2);

- компактификация Бора $\mathbb{R}_{B}^{n}, C^{*}$-алгебра $\mathscr{A} P\left(\mathbb{R}^{n}\right)$ (п. 15.1).

Авторы хотели бы поблагодарить Н. А. Азамова и А. А. Седаева за комментарии, помощь и критические замечания. 


\section{2. Предварительные сведения: пространства и функционалы}

Рассмотрим банахово пространство $\left(E,\|\cdot\|_{E}\right)$, состоящее из вещественнозначных измеримых по Лебегу функций (отождествляемых почти всюду) на интервале $J=[0, \infty)$ или на $J=\mathbb{N}$. Пусть $x^{*}$ обозначает невозрастающую непрерывную справа перестановку функции $|x|$, задаваемую формулой

$$
x^{*}(t)=\inf \{s \geqslant 0: \lambda(\{|x|>s\}) \leqslant t\}, \quad t>0,
$$

где $\lambda$ обозначает меру Лебега. Тогда $E$ будет называться перестановочно-инвариантным, если:

(i) $E$ является идеальной решеткой, т.е. из того, что $y \in E$ и $x$ - измеримая функция на $J$ с $0 \leqslant|x| \leqslant|y|$, следует, что $x \in E$ и $\|x\|_{E} \leqslant\|y\|_{E}$;

(ii) из того, что $y \in E$, а $x$ - измеримая функция на $J$, удовлетворяющая условию $x^{*}=y^{*}$, следует, что $x \in E$ и $\|x\|_{E}=\|y\|_{E}$.

В случае $J=\mathbb{N}$ удобно отождествлять $x^{*}$ с перестановкой последовательности $|x|=\left\{\left|x_{n}\right|\right\}_{n=1}^{\infty}$ в убывающем порядке. По поводу основных свойств перестановочно-инвариантных пространств мы отсылаем читателя к монографиям [42]-[44]. Заметим, что для любого перестановочно-инвариантного пространства $E=E(J)$ имеют место следующие непрерывные вложения:

$$
L_{1} \cap L_{\infty}(J) \subseteq E \subseteq L_{1}+L_{\infty}(J) .
$$

Будем говорить, что перестановочно-инвариантное пространство $E$ есть вполне симметричное банахово пространство, если оно обладает дополнительным свойством, заключающимся в том, что если $y \in E$ и $L_{1}+L_{\infty}(J) \ni x \prec \prec y$, то $x \in E$ и $\|x\|_{E} \leqslant\|y\|_{E}$. Здесь $x \prec \prec y$ обозначает мажорирование в смысле ХардиЛитлвуда-Пойя:

$$
\int_{0}^{t} x^{*}(s) d s \leqslant \int_{0}^{t} y^{*}(s) d s \quad \forall t>0 .
$$

Классический пример несепарабельных вполне симметричных пространств функций и последовательностей $E(J)$ дается пространствами Марцинкевича.

\section{1. Пространства Марцинкевича функций и последовательностей.} Пусть $\Omega$ обозначает множество таких вогнутых функций $\psi:[0, \infty) \rightarrow[0, \infty)$, что $\lim _{t \rightarrow 0^{+}} \psi(t)=0$ и $\lim _{t \rightarrow \infty} \psi(t)=\infty$. Важными примерами функций, принадлежащих множеству $\Omega$, являются функции $t, \log (1+t), t^{\alpha}$ и $(\log (1+t))^{\alpha}$ при $0<\alpha<1$. Пусть $\psi \in \Omega$. Определим функцию взвешенного среднего

$$
a(x, t)=\frac{1}{\psi(t)} \int_{0}^{t} x^{*}(s) d s, \quad t>0,
$$

и обозначим через $M(\psi)$ пространство Марцинкевича, состоящее из таких измеримых функций $x$ на $[0, \infty)$, что

$$
\|x\|_{M(\psi)}:=\sup _{t>0} a(x, t)=\|a(x, \cdot)\|_{\infty}<\infty .
$$

Определение пространства Марцинкевича последовательностей $\left(m(\psi),\|x\|_{m(\psi)}\right)$ аналогично:

$$
m(\psi)=\left\{x=\left\{x_{n}\right\}_{n=1}^{\infty}:\|x\|_{m(\psi)}:=\sup _{N \geqslant 1} \frac{1}{\psi(N)} \sum_{n=1}^{N} x_{n}^{*}<\infty\right\} .
$$


Замыкание по норме пространства $M(\psi) \cap L^{1}([0, \infty))$ (соответственно пространства $\left.\ell_{1}=\ell_{1}(\mathbb{N})\right)$ в $M(\psi)$ (соответственно в $m(\psi)$ ) обозначается через $M_{1}(\psi)$ (соответственно $\left.m_{1}(\psi)\right)$. Для любого $\psi \in \Omega$ мы имеем $M_{1}(\psi) \neq M(\psi)$. Банаховы пространства $\left(M(\psi),\|\cdot\|_{M(\psi)}\right),\left(m(\psi),\|\cdot\|_{m(\psi)}\right),\left(M_{1}(\psi),\|\cdot\|_{M(\psi)}\right),\left(m_{1}(\psi)\right.$, $\left.\|\cdot\|_{m(\psi)}\right)$ являются примерами вполне симметричных пространств [43], [42].

Пусть $M_{+}(\psi)$ (соответственно $m_{+}(\psi)$ ) обозначает множество положительных функций из $M(\psi)$ (соответственно из $m(\psi))$. Для любого $x \in M(\psi)$ будем писать $x=x_{+}-x_{-}$, где $x_{+}:=x \chi_{\{t: x(t)>0\}}$ и $x_{-}:=x-x_{+}$. Пространства

$$
\mathscr{L}^{(1, \infty)}:=M(\log (1+t)) \cap L_{\infty} \quad \text { и } \quad \mathscr{L}^{(p, \infty)}:=M\left(t^{1-\frac{1}{p}}\right) \cap L_{\infty}, \quad 1<p<\infty,
$$

играют в дальнейшем очень важную роль. Заметим, что эти пространства все еще являются пространствами Марцинкевича. Действительно, пространство $\mathscr{L}^{(1, \infty)}$ (соответственно $\mathscr{L}^{(p, \infty)}, p>1$ ) можно отождествить с пространством $M\left(\psi_{1}\right)$ (соответственно $M\left(\psi_{p}\right), p>1$ ), где

$$
\psi_{1}(t)=\left\{\begin{array}{ll}
t \cdot \log 2, & 0 \leqslant t \leqslant 1, \\
\log (1+t), & 1 \leqslant t<\infty,
\end{array} \quad \psi_{p}(t)=\left\{\begin{array}{ll}
t, & 0 \leqslant t \leqslant 1, \\
t^{1-\frac{1}{p}}, & 1 \leqslant t<\infty,
\end{array} \quad p>1\right.\right.
$$

Норма (Марцинкевича), задаваемая формулой $(2)$, на пространстве $\mathscr{L}^{(p, \infty)}$ обозначается через $\|\cdot\|_{(p, \infty)}, 1 \leqslant p<\infty$.

\section{2. Сингулярные симметричные функционалы на пространствах Марцинкевича.}

ОПРЕДЕЛЕНИЕ 2.1. Скажем, что положительный функционал $f \in M(\psi)^{*}$ симметричен (соответственно перестановочно-инвариантен), если $f(x) \leqslant f(y)$ для любых таких $x, y \in M_{+}(\psi)$, что $x \prec \prec y$ (соответственно $x^{*}=y^{*}$ ). Будем говорить, что такой функционал сосредоточен на бесконечности (или сингулярен), если $f(|x|)=0$ для любого $x \in M_{1}(\psi)$ (эквивалентно, $f\left(x^{*} \chi_{[0, s]}\right)=0$ для любого $x \in M(\psi)$ и характеристической функции $\chi_{[0, s]}$ интервала $[0, s]$ для любого $s>0$ ).

Следующая теорема полностью характеризует пространства Марцинкевича, допускающие нетривиальные симметричные функционалы.

Teоpema 2.2 [31], [40], [41]. Если $\psi \in \Omega$, mo

(i) ненулевой сосредоточенный на бесконечности симметричный функиионал на пространстве $M(\psi)$ (соответственно $m(\psi))$ существует тогда и только тогда, когда

$$
\liminf _{t \rightarrow \infty} \frac{\psi(2 t)}{\psi(t)}=1
$$

(ii) ненулевой сосредоточенный в нуле симметричный функиионал на пространстве $M(\psi)$ существует тогда и только тогда, когда

$$
\liminf _{t \downarrow 0} \frac{\psi(2 t)}{\psi(t)}=1
$$


Таким образом, например, $\left(\mathscr{L}^{(1, \infty)}\right)_{\mathrm{sym}, \infty}^{*} \neq\{0\}$, тогда как $\left(\mathscr{L}^{(p, \infty)}\right)_{\mathrm{sym}, \infty}^{*}=$ $\{0\}$ для любого $1<p<\infty$. Условия (3) и (4) допускают следующую геометрическую интерпретацию. Обозначим через $N(\psi)$ замыкание по норме в $M(\psi)$ (порядкового) идеала

$$
N(\psi)^{0}:=\left\{f \in M(\psi): f^{*}(\cdot) \leqslant k \psi^{\prime}(\cdot / k) \text { при некотором } k \in \mathbb{N}\right\} .
$$

Очевидно, что $N(\psi)$ - банахово перестановочно-инвариантное пространство (подпространство пространства $M(\psi)$ ). Предполагая (для простоты), что $\psi$ линейна в окрестности нуля, мы имеем, что пространство $N(\psi)$ вполне симметрично (и, тем самым, совпадает с $M(\psi)$ ) тогда и только тогда, когда не выполнено условие (3). Другими словами, $M(\psi)_{\mathrm{sym}, \infty}^{*} \neq\{0\}$ тогда и только тогда, когда $N(\psi) \neq M(\psi)$. По поводу этой и других геометрических интерпретаций условий (3) и (4) мы отсылаем читателя к работам [45], [42; II.5.7] и [46]. По поводу различных конструкций сингулярных симметричных функционалов на $M(\psi)$ (и, более общим образом, на вполне симметричных пространствах и их некоммутативных аналогах) мы отсылаем читателя к работам [31], [40], [41]. Обзор конструкций, имеющих отношение к нашей главной теме, будет дан ниже, в разделе 5 .

Наше особое внимание к сосредоточенным на бесконечности симметричным функционалам объясняется многочисленными приложениями их аналогов в некоммутативной геометрии. Некоммутативные аналоги сосредоточенных в нуле симметричных функционалов можно рассматривать как "следы Диксмье, ассоциированные с алгебрами фон Неймана типа $I I_{1}$ ”, но к настоящему времени им не найдено ни одного приложения.

\section{3. Симметричные операторные пространства и функционалы.}

Сейчас мы распространим идеи предыдущих пунктов на (некоммутативные) пространства измеримых операторов. Мы обозначим через $\mathscr{N}$ полуконечную алгебру фон Неймана в гильбертовом пространстве $\mathscr{H}$ с фиксированным точным и нормальным полуконечным следом $\tau$. В основном мы будем иметь дело со случаем, когда $\tau(1)=\infty$, где 1 - единица в $\mathscr{N}$. Линейный оператор $x: \operatorname{dom}(x) \rightarrow \mathscr{H}$ с областью определения $\operatorname{dom}(x) \subseteq \mathscr{H}$ называется присоединенным к $\mathscr{N}$, если $u x=x u$ для всех унитарных элементов $u$ в коммутанте $\mathscr{N}^{\prime}$ алгебры $\mathscr{N}$. Замкнутый и плотно определенный оператор $x$, присоединенный к $\mathscr{N}$, называется $\tau$-измеримым, если для любого $\varepsilon>0$ существует такой ортогональный проектор $p \in \mathscr{N}$, что $p(\mathscr{H}) \subseteq \operatorname{dom}(x)$ и $\tau(1-p)<\varepsilon$. Множество всех $\tau$-измеримых операторов обозначается через $\widetilde{\mathscr{N}}$.

Напомним понятие функции обобщенных сингулярных значений [47], [48]. Для заданного самосопряженного оператора $x$ в $\mathscr{H}$ обозначим через $e^{x}(\cdot)$ его спектральную меру. Предположим, что $x$ является $\tau$-измеримым. Тогда $e^{|x|}(B) \in \mathscr{N}$ для всех борелевских множеств $B \subseteq \mathbb{R}$ и существует такое $s>0$, что $\tau\left(e^{|x|}(s, \infty)\right)<\infty$. Для $t \geqslant 0$ определим

$$
\mu_{t}(x)=\inf \left\{s \geqslant 0: \tau\left(e^{|x|}(s, \infty)\right) \leqslant t\right\}
$$

Функция $\mu(x):[0, \infty) \rightarrow[0, \infty]$ называется функцией обобщенных сингулярных значений (или убывающей перестановкой) оператора $x$; заметим, что $\mu_{t}(x)<$ $\infty$ для всех $t>0$. По поводу основных свойств этой функции сингулярных значений мы отсылаем читателя к работе [47]. 
Если мы рассмотрим пространство $\mathscr{N}=L_{\infty}([0, \infty), m)$, где $m$ обозначает меру Лебега на $[0, \infty)$, как абелеву алгебру фон Неймана, действующую посредством умножения в гильбертовом пространстве $\mathscr{H}=L^{2}([0, \infty), m)$, со следом $\tau$, задаваемым интегрированием по мере $m$, то легко видеть, что множество всех $\tau$-измеримых операторов, присоединенных к $\mathscr{N}$, состоит из всех измеримых функций на $[0, \infty)$, которые ограничены вне некоторого множества конечной меры, и что функция обобщенных сингулярных значений $\mu(f)$ в точности есть убывающая перестановка $f^{*}$.

Если $\mathscr{N}=\mathscr{L}(\mathscr{H})\left(\right.$ соответственно $\left.\ell_{\infty}(\mathbb{N})\right)$ и $\tau-$ стандартный след $\operatorname{Tr}($ соответственно считающая мера на $\mathbb{N}$ ), то нетрудно видеть, что $\widetilde{\mathscr{N}}=\mathscr{N}$. В этом случае $x \in \mathscr{N}$ компактен тогда и только тогда, когда $\lim _{t \rightarrow \infty} \mu_{t}(x)=0$; более того,

$$
\mu_{n}(x)=\mu_{t}(x), \quad t \in[n, n+1), \quad n=0,1,2, \ldots,
$$

и последовательность $\left\{\mu_{n}(x)\right\}_{n=0}^{\infty}$ есть в точности последовательность собственных значений элемента $|x|$, взятая в невозрастающем порядке с учетом кратностей.

Для полуконечной алгебры фон Неймана $(\mathscr{N}, \tau)$ и вполне симметричного банахова функционального пространства $\left(E,\|\cdot\|_{E}\right)$ на $([0, \infty), m)$ мы определим соответствующее некоммутативное пространство $E(\mathscr{N}, \tau)$, полагая

$$
E(\mathscr{N}, \tau)=\{x \in \widetilde{\mathscr{N}}: \mu(x) \in E\} .
$$

Наделенное нормой $\|x\|_{E(\mathscr{N}, \tau)}:=\|\mu(x)\|_{E}$ пространство $\left(E(\mathscr{N}, \tau),\|\cdot\|_{E(\mathscr{N}, \tau)}\right)$ является банаховым пространством и называется (некоммутативным) вполне симметричным операторным пространством на $(\mathscr{N}, \tau)$, порожденным пространством $\left(E,\|\cdot\|_{E}\right)$. Если $\mathscr{N}=\ell_{\infty}(\mathbb{N})$, то пространство $E(\mathscr{N}, \tau)$ просто есть (вполне) симметричное пространство последовательностей $\ell_{E}$, которое можно рассматривать как линейную оболочку в $E$ векторов $e_{n}=\chi_{[n-1, n)}, n \geqslant 1$ (см., например, [43]). В случае $(\mathscr{N}, \tau)=(\mathscr{L}(\mathscr{H}), \operatorname{Tr})$ мы обозначим $E(\mathscr{N}, \tau)$ просто через $E(\mathscr{H})$. Заметим, что последнее пространство совпадает с (симметрично-нормированным) идеалом компактных операторов в $\mathscr{H}$, ассоциированных с (симметричным) пространством последовательностей $\ell_{E}$ (см., например, [49]).

В основном мы будет иметь дело с вполне симметричными операторными пространствами $E(\mathscr{N}, \tau)$ и $E(\mathscr{H})$, когда $E=M(\psi)$, в частности, когда $E=\mathscr{L}^{(p, \infty)}, 1 \leqslant p<\infty$. Мы ссылаемся на пространства $M(\psi)(\mathscr{N}, \tau)$ как на операторные пространства Марцинкевича. Иногда, для краткости, мы будем опускать символы $(\mathscr{N}, \tau)$ и $\mathscr{H}$ в обозначениях, и это не должно вызывать путаницы.

Дальнейшие ссылки, касающиеся теории вполне симметричных операторных пространств, можно найти в работах [31], [50], [51].

ОПРЕДЕЛЕНИЕ 2.3. Линейный функционал $\varphi \in E(\mathscr{N}, \tau)^{*}$ называется симметричным (соответственно перестановочно-инвариантным), если $\varphi$ положителен (т.е. $\varphi(x) \geqslant 0$ при $0 \leqslant x \in E(\mathscr{N}, \tau))$ и $\varphi(x) \leqslant \varphi(y)$, если $\mu(x) \prec \prec \mu(y)$ (соответственно $\varphi(x)=\varphi(y)$, если $x, y \geqslant 0$ и $\mu(x)=\mu(y)$ ).

Для данного $x \in \widetilde{\mathscr{N}}$ множество $\Omega(x)=\{y \in \widetilde{\mathscr{N}}: y \prec \prec x\}$ называется орбитой оператора $x$. Если $x \in L_{1}(\mathscr{N}, \tau)+\mathscr{N}$, то множество $\Omega(x)$ удобно описывается в терминах абсолютных сжатий. Обозначим через $\Sigma$ множество 
всех линейных операторов $T: L_{1}(\mathscr{N}, \tau)+\mathscr{N} \rightarrow L_{1}(\mathscr{N}, \tau)+\mathscr{N}$ со свойствами: (a) $T(a) \in L_{1}(\mathscr{N}, \tau)$ (соответственно $\mathscr{N}$ ), если $a \in L_{1}(\mathscr{N}, \tau)$ (соответственно $\mathscr{N})$, (b) $\|T\|_{L_{1}(\mathscr{N}, \tau) \rightarrow L_{1}(\mathscr{N}, \tau)} \leqslant 1,\|T\|_{\mathscr{N} \rightarrow \mathscr{N}} \leqslant 1$. Из [52] следует, что соотношение $y \prec \prec x, x \in L_{1}(\mathscr{N}, \tau)+\mathscr{N}, y \in \widetilde{\mathscr{N}}$, справедливо тогда и только тогда, когда существует такой оператор $T \in \Sigma$, что $T(x)=y$. Таким образом,

$$
\Omega(x)=\{T x: T \in \Sigma\} .
$$

Если $E(\mathscr{N}, \tau)$ - вполне симметричное операторное пространство, то мы имеем $\Omega(x) \subseteq E(\mathscr{N}, \tau)$ для каждого $x \in E(\mathscr{N}, \tau)$, и поэтому ограниченный положительный линейный функционал $\varphi$ на $E(\mathscr{N}, \tau)$ симметричен тогда и только тогда, когда $\varphi(|T x|) \leqslant \varphi(x)$ для любых $T \in \Sigma$ и $0 \leqslant x \in E(\mathscr{N}, \tau)$.

Теперь мы предположим, что $\alpha: \mathscr{N} \rightarrow \mathscr{N}-*$-автоморфизм, который, кроме того, сохраняет след, т.е. $\tau(\alpha(a))=\tau(a)$ для любого $0 \leqslant a \in \mathscr{N}$. Легко видеть, что такой автоморфизм продолжается (единственным образом) до *-автоморфизма $\widetilde{\alpha}: \widetilde{\mathscr{N}} \rightarrow \widetilde{\mathscr{N}}$, который инвариантен при перестановках, т.е. $\mu(\widetilde{\alpha}(x))=\mu(x)$ для любого $x \in \widetilde{\mathscr{N}}$. Таким образом, мы можем рассматривать перестановочно-инвариантные функционалы на $E(\mathscr{N}, \tau)$ как положительные функционалы, которые инвариантны под действием группы всех сохраняющих след *-автоморфизмов алгебры $\mathscr{N}$, являющейся подгруппой в $\Sigma$. Если $\mathscr{N}=\mathscr{L}(\mathscr{H})$, то каждый перестановочно-инвариантный функционал на $E(\mathscr{H})$ является просто следом (т.е. унитарно инвариантным положительным функционалом на $\mathscr{L}(\mathscr{H}))$, продолжающимся до непрерывного линейного функционала на $E(\mathscr{H})$. Очевидно, что каждый симметричный функционал является перестановочно-инвариантным. Однако априори непонятно (см. [37]), существует ли (например, на идеале $\left.\mathscr{L}^{(1, \infty)}(\mathscr{H})\right)$ перестановочно-инвариантный сингулярный функционал, который не обязательно симметричен, или существует ли след на $\mathscr{L}^{(1, \infty)}(\mathscr{H})$, который не совпадает со следом Диксмье (см. ниже раздел 5). Первый пример такого следа появился совсем недавно в работе [35]. Фактически, если

$$
\lim _{t \rightarrow \infty} \frac{\psi(2 t)}{\psi(t)}=1,
$$

то на каждом операторном пространстве Марцинкевича $M(\psi)(\mathscr{N}, \tau)$ существует ненулевой след (или перестановочно-инвариантный функционал), равный нулю на $N(\psi)(\mathscr{N}, \tau)$. В частности, если $\psi(t)=\log (1+t)$, то такой функционал обращается в нуль на каждом операторе $0 \leqslant x \in \mathscr{L}^{(1, \infty)}(\mathscr{N}, \tau)$ таком, что $\mu_{t}(x)=1 / t$ (или, если $\mathscr{N}=B(\mathscr{H})$, на каждом компактном операторе $0 \leqslant x \in \mathscr{L}^{(1, \infty)}(\mathscr{H})$ таком, что $\left.\mu_{n}(x)=1 / n, n \geqslant 1\right)$. До сих пор неизвестно, существуют ли перестановочно-инвариантные функционалы, которые не симметричны, на каждом $M(\psi)(\mathscr{N}, \tau)$, где $\psi$ удовлетворяет условию $(3)$.

\section{3. Общие факты о симметричных функционалах}

Пусть $E=E(0, \infty)$ - вполне симметричное пространство. Обозначим через $E_{+}$множество всех неотрицательных функций из $E$ и через $E_{\text {sym }}^{*}$ множество всех симметричных функционалов на $E$.

Положительный линейный функционал $\varphi$ на $E$ называется нормалъным (или поряджово непрерывным), если из того, что $f_{n} \downarrow 0$, следует, что $\varphi\left(f_{n}\right) \downarrow 0$. 
ПрЕДЛОЖЕНИЕ 3.1 [31]. Если функиионал $\varphi \in E_{\mathrm{sym}}^{*}$ порядково непрерывен, то $E \subset L^{1}[0, \infty)$ и $\varphi$ пропориионален интегралу по мере Лебега.

Мы определим оператор растяжения $D_{s}$, как в работе [42], по формуле $D_{s} f(t)=f(t / s)$. Заметим, что $D_{s}$ является ограниченным оператором в $E$ и $\left\|D_{s}\right\|_{E \rightarrow E} \leqslant \max \{1, s\}$, более того, $\left(D_{s} f\right)^{*}=D_{s} f^{*}$ для любой функции $f \in$ $E$. Следующий результат установлен в [31; предложение 2.3] при предположении, что $\varphi$ симметричен, однако доказательство справедливо также для перестановочно-инвариантных функционалов.

ПрЕДЛОЖЕНИЕ 3.2. Если $0 \leqslant \varphi \in E^{*}$ перестановочно-инвариантен, то $\varphi\left(D_{s} f\right)=s \varphi(f)$ для любих $f \in E u s>0$.

Положительный элемент $\varphi \in E^{*}$ называется сингулярным, если из того, что $0 \leqslant \varphi^{\prime} \leqslant \varphi, \varphi^{\prime} \in E^{*}$ и $\varphi^{\prime}$ порядково непрерывен, следует $\varphi^{\prime}=0$.

ПРЕДЛОЖЕНИЕ 3.3 [31].

(i) Каждый симметричный функиионал на $E$ можно представить единственным образом в виде суммы нормалъного функционала и сингулярного симметричного функционала. Более того, нормалъный функционал равен нулю, если условие $E \subseteq L^{1}[0, \infty)$ не выполнено.

(ii) Любой сингулярный симметричный функционал можно единственным образом представить в виде суммы сингулярных симметричных функционалов, сосредоточенных в нуле и на бесконечности.

(iii) Множество симметричных функиионалов образует решетку.

Следующий результат показывает, что каждый симметричный функционал на $E$ допускает “естественное продолжение” до симметричного функционала на $E(\mathscr{N}, \tau)$ для любой полуконечной алгебры фон Неймана $(\mathscr{N}, \tau)$. Обозначим через $E(\mathscr{N}, \tau)+$ множество всех положительных операторов из $E(\mathscr{N}, \tau)$.

Tеорема 3.4 [31]. Пусть $\varphi_{0} \in E_{\text {sym }}^{*} u \varphi(x):=\varphi_{0}(\mu(x))$ для любого $x \in$ $E(\mathscr{N}, \tau)_{+}$. Тогда $\varphi$ продолжается до симметричного функиионала $0 \leqslant \varphi \in$ $E(\mathscr{N}, \tau)^{*}$.

ДокАЗАТЕЛЬСтво. Понятно, что достаточно доказать, что $\varphi$ аддитивен на $E(\mathscr{N}, \tau)_{+}$. Пусть $x, y \in E(\mathscr{N}, \tau)_{+}$. Из того, что $\mu(x+y) \prec \prec \mu(x)+\mu(y)[47 ;$ теорема 4.4] и $\varphi_{0}$ симметричен, следует, что

$$
\varphi(x+y)=\varphi_{0}(\mu(x+y)) \leqslant \varphi_{0}(\mu(x)+\mu(y))=\varphi(x)+\varphi(y) .
$$

Для того чтобы доказать обратное неравенство, мы используем легко проверяемый факт (см., например, [33; предложение 1.10]), что

$$
\int_{0}^{t} \mu_{s}(x) d s+\int_{0}^{t} \mu_{s}(y) d s \leqslant \int_{0}^{2 t} \mu_{s}(x+y) d s \quad \forall t>0 .
$$

Замечая, что (6) эквивалентно мажорированию

$$
\mu(x)+\mu(y) \prec \prec 2 D_{\frac{1}{2}} \mu(x+y),
$$

из предложения 3.2 получаем, что

$$
\varphi(x)+\varphi(y)=\varphi_{0}(\mu(x)+\mu(y)) \leqslant 2 \varphi_{0}\left(D_{\frac{1}{2}} \mu(x+y)\right)=\varphi_{0}(\mu(x+y))=\varphi(x+y) .
$$


Таким образом, $\varphi$ аддитивен на $E(\mathscr{N}, \tau)_{+}$, и этого достаточно, чтобы завершить доказательство теоремы.

Теорема 3.5. Пусть $(\mathscr{N}, \tau)$ - полуконечная алгебра фон Неймана, не имеющая минимальных проекторов, и пусть $E$ - вполне симметричное банахово функциональное пространство на $[0, \infty)$. Если $0 \leqslant \varphi \in E(\mathscr{N}, \tau)^{*}-$ cuмметричный функиионал, то существует такой симметричный функиионал $0 \leqslant \varphi_{0} \in E^{*}$, ито $\varphi(x)=\varphi_{0}(\mu(x))$ для любого $0 \leqslant x \in E(\mathscr{N}, \tau)$.

ДокАЗАтЕЛЬство. Достаточно показать, что существует симметричный функционал $0 \leqslant \varphi_{0} \in E[0, \tau(1))^{*}$, удовлетворяющий условию $\varphi(x)=\varphi_{0}(\mu(x))$ для любого $0 \leqslant x \in E(\mathscr{N}, \tau)$. Пусть $\mathscr{M}$ - коммутативная алгебра фон Неймана $L_{\infty}[0, \tau(1))$, наделенная следом, задаваемым интегрированием. Алгебру $\widetilde{\mathscr{M}}$ можно отождествить с пространством всех измеримых функций на $[0, \tau(1))$, которые ограничены вне множества конечной меры. Поскольку $\mathscr{M}$ не содержит минимальных проекторов, существует положительный сохраняющий перестановки гомоморфизм алгебр $J: \widetilde{\mathscr{M}} \rightarrow \widetilde{\mathscr{N}}$ [51; лемма 4.1], [53; теорема 3.5]. Рассмотрим симметричный функционал $0 \leqslant \varphi \in E(\mathscr{N}, \tau)^{*}$. Для $f \in E[0, \tau(1))$ определим $\varphi_{0}(f):=\varphi(J f)$. Понятно, что $0 \leqslant \varphi_{0} \in E[0, \tau(1))^{*}$ симметричен. Более того, если $0 \leqslant x \in E(\mathscr{N}, \tau)$, то $\mu(J(\mu(x)))=\mu(x)$ и, следовательно, $\varphi(x)=\varphi(J(\mu(x)))=\varphi_{0}(\mu(x))$. Теорема 3.5 доказана.

Еще легче увидеть, что соответствие между множеством симметричных функционалов на пространстве $E$ и множеством симметричных функционалов на пространстве $E(\mathscr{N}, \tau)$, приведенное в теоремах 3.4 и 3.5 , также существует для множеств симметричных функционалов $\left(\ell_{E}\right)_{\mathrm{sym}}^{*}$ и $(E(\mathscr{H}))_{\mathrm{sym}}^{*}$. Более того, как показывает следующий результат, существует простая связь между множествами $\left(\ell_{E}\right)_{\mathrm{sym}}^{*}$ и $E_{\mathrm{sym}}^{*}$.

ТеОРема 3.6 [31]. Пусть Е - вполне симметричное банахово функциональное пространство на $[0, \infty)$ и $E(\mathscr{H})$ - соответствующий идеал компактных операторов в бесконечномерном гилъбертовом пространстве $\mathscr{H}$. Если $0 \leqslant \varphi \in E(\mathscr{N}, \tau)^{*}$ - симметричный функиионал, то существует $\varphi_{0} \in E_{\text {sуm }}^{*}$ такой, что $\varphi(x)=\varphi_{0}(\mu(x))$ для любых $x \in E(\mathscr{N}, \tau)_{+}$.

Теперь рассмотрим следующий вопрос, естественно возникающий из теоремы 3.4 и предложенный в работе [37]. Предположим, что $E$ (соответственно $\ell_{E}$ ) есть вполне симметричное функциональное пространство (соответственно пространство последовательностей) и $\varphi_{0}$ - положительный перестановочно-инвариантный функционал на $E$ (соответственно $\left.\ell_{E}\right)$. Является ли функционал

$$
\varphi(x):=\varphi_{0}(\mu(x))
$$

аддитивным на $E(\mathscr{N}, \tau)$ (соответственно $E(\mathscr{H}))$ ? Совсем недавно в [35] был получен утвердительный ответ на этот вопрос при условии, что $\mathscr{N}$ - фактор. Доказательство, приведенное в [35], опирается на глубокие результаты из работы [32] (см. также [54], [55]), касающиеся структуры пространства коммутаторов $[I, J]$, порожденного всеми коммутаторами $[T, S], T \in I, S \in J$ (здесь $I$ и $J$ - идеалы компактных операторов в $\mathscr{L}(\mathscr{H})$ ). Заметим, что каждый перестановочно-инвариантный функционал на идеале $I$ обращается в нуль на $[I, \mathscr{L}(\mathscr{H})]$. Много важных результатов из [32], [54], [55] допускают обобщение на случай общих полуконечных алгебр фон Неймана и их идеалов [56], [57]. 


\section{4. Предварительные сведения о состояниях, инвариантных при растяжениях и сдвигах}

Конструкция следов Диксмье $\tau_{\omega}$ связана с выбором "инвариантного среднего" $\omega$. В этом разделе мы дадим обзор важнейших классов таких средних. Обозначим через $\ell_{\infty}=\ell_{\infty}(\mathbb{N})$ банахово пространство всех ограниченных последовательностей комплексных чисел. Под состоянием на унитальной $C^{*}$-алгебре мы понимаем положительный линейный функционал, принимающий значение 1 на единице алгебры. Напомним, что положительный линейный функционал $\mathbf{L} \in \ell_{\infty}^{*}$ называется банаховым пределом, если $\mathbf{L}$ инвариантен относительно сдвигов и $\mathbf{L}(\mathbf{1})=1$ (здесь $\mathbf{1}=(1,1,1, \ldots)$ ). Банахов предел $\mathbf{L}$, в частности, удовлетворяет условию $\mathbf{L}(\xi)=0$ для любого $\xi \in c_{0}\left(c_{0}-\right.$ все последовательности из $\ell_{\infty}$, сходящиеся к нулю). Мы обозначим совокупность всех банаховых пределов на $\ell_{\infty}$ через $B L(\mathbb{N})$. Заметим, что $\|\mathbf{L}\|=1$ для любого $\mathbf{L} \in B L(\mathbb{N})$.

Напомним, что последовательность $\xi=\left\{\xi_{n}\right\}_{n=1}^{\infty} \in \ell_{\infty}$ называется почти сходящейся к $\alpha \in \mathbb{R}$ (обозначение: $F-\lim _{n \rightarrow \infty} \xi_{n}=\alpha$ ) тогда и только тогда, когда $\mathbf{L}(\xi)=\alpha$ для всех $\mathbf{L} \in B L(\mathbb{N})$. Понятие почти сходящейся последовательности введено Г. Г. Лоренцем [58], который показал, что последовательность $\left\{\xi_{n}\right\}_{n=1}^{\infty}$ почти сходится к $\alpha$ тогда и только тогда, когда $\lim _{p \rightarrow \infty} \frac{\xi_{n}+\xi_{n+1}+\cdots+\xi_{n+p-1}}{p}$ $=\alpha$ равномерно по $n=1,2, \ldots$. Обозначим через $a c$ (соответственно $\left.a c_{0}\right)$ множество всех почти сходящихся (соответственно всех почти сходящихся к 0) последовательностей из $\ell_{\infty}$. Очевидно, что $a c$ и $a c_{0}$ являются замкнутыми подпространствами в $\ell_{\infty}$. Мы определим оператор сдвига $T: \ell_{\infty} \rightarrow \ell_{\infty}$, оператор Чезаро $H: \ell_{\infty} \rightarrow \ell_{\infty}$ и операторы растяжения $D_{n}: \ell_{\infty} \rightarrow \ell_{\infty}$ при $n \in \mathbb{N}$ по формулам

$$
\begin{aligned}
T\left(x_{1}, x_{2}, x_{3}, \ldots\right) & =\left(x_{2}, x_{3}, x_{4}, \ldots\right), \\
H\left(x_{1}, x_{2}, x_{3}, \ldots\right) & =\left(x_{1}, \frac{x_{1}+x_{2}}{2}, \frac{x_{1}+x_{2}+x_{3}}{3}, \ldots\right), \\
D_{n}\left(x_{1}, x_{2}, x_{3}, \ldots\right) & =(\underbrace{x_{1}, \ldots, x_{1}}_{n}, \underbrace{x_{2}, \ldots, x_{2}}_{n}, \ldots)
\end{aligned}
$$

для любого $x=\left(x_{1}, x_{2}, x_{3}, \ldots\right) \in \ell_{\infty}$.

Каждый из введенных выше операторов положителен и сохраняет единичный элемент 1 алгебры $\ell_{\infty}$ и, следовательно, ограничен с нормой, равной 1 . Более того, $\left\{D_{n}\right\}_{n=1}^{\infty}$ является абелевой полугруппой. Главным инструментом в нашей конструкции различных классов инвариантных средних является хорошо известная теорема Маркова-Какутани о неподвижной точке.

Теорема 4.1 (Марков-Какутани). Пусть $F$ - локально выпуклое хаусдорфово пространство и $K$ - непустое выпуклое компактное подмножество в $F$. Пусть $\mathscr{T}$ - такая абелева полугруппа линейных непрерывных операторов в $F$, что $S(K) \subseteq K$ для любого $S \in \mathscr{T}$. Тогда существует такой элемент $x \in K$, что $S x=x$ для любого $S \in \mathscr{T}$.

Легко видеть, что множество всех неподвижных точек в теореме 4.1 образует выпуклое компактное подмножество в $K$. 
Мы будем применять теорему Маркова-Какутани в случае пространств $F=$ $\left(\ell_{\infty}\right)^{*},\left(L_{\infty}(\mathbb{R})\right)^{*},\left(L_{\infty}\left(\mathbb{R}_{+}^{*}\right)\right)^{*}$, наделенных слабой *-топологией. Для простоты изложения мы приведем доказательства только в первом случае.

Лемма 4.2 [41], [29]. Справедливы следующие утверждения:

(i) $D_{n} T=T^{n} D_{n} \quad \forall n \geqslant 1$;

(ii) $H T x-T H x \in c_{0} \quad \forall x \in \ell_{\infty}$;

(iii) $H D_{n} x-D_{n} H x \in c_{0} \quad \forall x \in \ell_{\infty}$.

ДокАзАтельство. Доказательство (i) получается непосредственно. Для доказательства (ii) заметим, что для любого $x \in \ell_{\infty}$

$$
\left|(H T x)_{k}-(T H x)_{k}\right|=\left|\frac{1}{k+1} \frac{x_{2}+\cdots+x_{k+1}}{k}-\frac{1}{k+1} x_{1}\right| \leqslant \frac{2}{k+1}\|x\|_{\infty},
$$

что показывает, что $H T x-T H x \in c_{0}$.

Теперь докажем (iii). Пусть $n \geqslant 1$ и $x \in \ell_{\infty}$. При $1 \leqslant k \in \mathbb{N}$ существуют такие $l \geqslant 1$ и $1 \leqslant r \leqslant n$, что $k=(l-1) n+r$. Следовательно,

$$
\left(H D_{n} x\right)_{k}=\frac{1}{k} \sum_{i=1}^{k}\left(D_{n} x\right)_{i}=\frac{n}{k} \sum_{j=1}^{l-1} x_{j}+\frac{r}{k} x_{l}
$$

и

$$
\left(D_{n} H x\right)_{k}=(H x)_{l}=\frac{1}{l} \sum_{j=1}^{l} x_{j} .
$$

Замечая, что $n l-k=n-r \leqslant n$ и $r l-k \leqslant n l-k \leqslant n$, получаем

$$
\begin{aligned}
\left|\left(H D_{n} x\right)_{k}-\left(D_{n} H x\right)_{k}\right| & =\left|\frac{n l-k}{k l} \sum_{j=1}^{l-1} x_{j}+\frac{r l-k}{k l} x_{l}\right| \\
& \leqslant \frac{n}{k}\left(\frac{1}{l} \sum_{j=1}^{l-1}\left|x_{j}\right|\right)+\frac{n}{k}\left|x_{l}\right| \leqslant \frac{2 n}{k}\|x\|_{\infty} .
\end{aligned}
$$

Это показывает, что $H D_{n} x-D_{n} H x \in \ell^{(1, \infty)} \subseteq c_{0}$. Лемма 4.2 доказана.

Теорема 4.3. Существует такое состояние $\widetilde{\omega}$ на $\ell_{\infty}$, что для любого $n \geqslant 1$

$$
\widetilde{\omega} \circ T=\widetilde{\omega} \circ H=\widetilde{\omega} \circ D_{n}=\widetilde{\omega} .
$$

ДоказАтельство. Положим $K=\left\{0 \leqslant \varphi \in\left(\ell_{\infty}\right)^{*}: \varphi(\mathbf{1})=1, T^{*} \varphi=\varphi\right\}$. Поскольку $K$ содержит обычные банаховы пределы, оно непусто. Очевидно, что $K$ выпукло и *-слабо компактно. Мы утверждаем, что $D_{n}^{*}(K) \subseteq K$. В самом деле, из приведенной выше леммы 4.2 (i) мы знаем, что $T^{*} D_{n}^{*}=D_{n}^{*}\left(T^{*}\right)^{n}$, следовательно, для $\varphi \in K$

$$
T^{*}\left(D_{n}^{*} \varphi\right)=D_{n}^{*}\left(T^{*}\right)^{n} \varphi=D_{n}^{*} \varphi
$$

откуда следует, что $D_{n}^{*} \varphi \in K$. Поэтому мы можем применить теорему 4.1 к множеству $K$ и абелевой полугруппе $\left\{D_{j}^{*}\right\}_{j=1}^{\infty}$. Следовательно, множество

$$
K_{1}=\left\{0 \leqslant \varphi \in\left(\ell_{\infty}\right)^{*}: \varphi(\mathbf{1})=1, T^{*} \varphi=\varphi, D_{n}^{*} \varphi=\varphi, n \geqslant 1\right\}
$$


непусто, и опять же очевидно, что $K_{1}$ выпукло и $*$-слабо компактно.

Теперь мы покажем, что $H^{*}\left(K_{1}\right) \subseteq K_{1}$. Для этого сначала заметим, что $\varphi(z)=0$ для любого $z \in c_{0}$ и любого $\varphi \in K_{1}\left(\right.$ так как $\left.T^{*} \varphi=\varphi\right)$. Для заданного $\varphi \in K_{1}$ из леммы 4.2 (iii) следует, что

$$
\left(D_{n}^{*} H^{*} \varphi\right)(x)-\left(H^{*} D_{n}^{*} \varphi\right)(x)=\varphi\left(H D_{n} x-D_{n} H x\right)=0
$$

для любого $x \in \ell_{\infty}$ и поэтому

$$
D_{n}^{*}\left(H^{*} \varphi\right)=H^{*}\left(D_{n}^{*} \varphi\right)=H^{*} \varphi
$$

Аналогично из леммы 4.2 (ii) следует, что $T^{*}\left(H^{*} \varphi\right)=H^{*} \varphi$ для любого $\varphi \in$ $K_{1}$. Следовательно, $H^{*}\left(K_{1}\right) \subseteq K_{1}$. Применяя теорему 4.1 к множеству $K_{1}$ и полугруппе $\left\{\left(H^{*}\right)^{n}\right\}_{n=0}^{\infty}$, мы можем заключить, что существует такой элемент $\widetilde{\omega} \in K_{1}$, что $H^{*}(\widetilde{\omega})=\widetilde{\omega}$, что завершает доказательство. Теорема 4.3 доказана.

Группу (по умножению) всех положительных действительных чисел мы обозначим $\mathbb{R}_{+}^{*}$.

Мы определим изоморфизм $L: L_{\infty}(\mathbb{R}) \rightarrow L_{\infty}\left(\mathbb{R}_{+}^{*}\right)$ по формуле $L(f)=f$ 。 $\log$ и средние (преобразования) Чезаро на $L_{\infty}(\mathbb{R})$ и $L_{\infty}\left(\mathbb{R}_{+}^{*}\right)$ соответственно по формулам:

$$
H(f)(u)=\frac{1}{u} \int_{0}^{u} f(v) d v \quad \text { для } \quad f \in L_{\infty}(\mathbb{R}), \quad u \in \mathbb{R},
$$

и

$$
M(g)(t)=\frac{1}{\log t} \int_{1}^{t} g(s) \frac{d s}{s} \quad \text { для } \quad g \in L_{\infty}\left(\mathbb{R}_{+}^{*}\right), \quad t>0 .
$$

Краткое вычисление дает для $g \in L_{\infty}\left(\mathbb{R}_{+}^{*}\right)$

$$
L H L^{-1}(g)(r)=\frac{1}{\log r} \int_{0}^{\log r} g\left(e^{u}\right) d u=\frac{1}{\log r} \int_{1}^{r} g(v) \frac{d v}{v}=M(g)(r),
$$

т.е. $L$ сплетает эти два средних.

Теперь мы рассмотрим аналоги операторов $T, D_{n}$ и $H$, действующие на $L_{\infty}(\mathbb{R})$ и $L_{\infty}\left(\mathbb{R}_{+}^{*}\right)$.

ОПРЕДЕЛЕНИЕ 4.4. $T_{b}$ обозначает сдвиг на $b \in \mathbb{R}, D_{a}$ обозначает растяжение на $\frac{1}{a} \in \mathbb{R}_{+}^{*}$ и $P^{a}$ обозначает возведение в степень $a \in \mathbb{R}_{+}^{*}$. То есть

$$
\begin{array}{lll}
T_{b}(f)(x)=f(x+b) & \text { для } & f \in L_{\infty}(\mathbb{R}), \\
D_{a}(f)(x)=f\left(\frac{x}{a}\right) & \text { для } & f \in L_{\infty}(\mathbb{R}), \\
P^{a}(f)(x)=f\left(x^{a}\right) & \text { для } & f \in L_{\infty}\left(\mathbb{R}_{+}^{*}\right) .
\end{array}
$$

Некоторые основные соотношения между этими $L_{\infty}$-пространствами и их отображениями в себя легко получаются из следующего предложения, доказательство которого аналогично доказательству леммы 4.2. 
ПРЕДЛОЖЕНИЕ 4.5 [29]. Пространство $L_{\infty}(\mathbb{R})$ вместе со своими отображениями в себя $D_{a}, T_{b}$ и $H(a>0, b \in \mathbb{R})$ связано с пространством $L_{\infty}\left(\mathbb{R}_{+}^{*}\right)$ вместе с его отображениями в себя $P^{a}, D_{a}$ и $M(a>0)$ при помощи изоморфизма

$$
L: L_{\infty}(\mathbb{R}) \rightarrow L_{\infty}\left(\mathbb{R}_{+}^{*}\right)
$$

и следующих тождеств:

(1) $L D_{\frac{1}{a}} L^{-1}=P^{a}$ nри $a>0$,

(2) $L T_{b} L^{-1}=D_{(\exp (b))^{-1}}$ npu $b \in \mathbb{R}$,

(3) $\mathrm{LHL}^{-1}=\mathrm{M}$,

(4) $D_{a} H=H D_{a}$ u $P^{a} M=M P^{a}$ nрu $a>0$,

(5) $\lim _{t \rightarrow \infty}\left(H T_{b}-T_{b} H\right) f(t)=0$ npu $f \in L_{\infty}(\mathbb{R}) u b \in \mathbb{R}$,

(6) $\lim _{t \rightarrow \infty}\left(M D_{a}-D_{a} M\right) f(t)=0$ npu $f \in L_{\infty}\left(\mathbb{R}_{+}^{*}\right) u a>0$.

ПРЕДЛОЖЕНИЕ 4.6 [29]. Если непрерывный функиионал $\widetilde{\omega} н а L_{\infty}(\mathbb{R})$ инвариантен под действием оператора Чезаро $H$, оператора сдвига $T_{a}$ или оператора растяжения $D_{a}$, то $\widetilde{\omega} \circ L^{-1}$ является непрерывным функционалом на $L_{\infty}\left(\mathbb{R}_{+}^{*}\right)$, инвариантным под действием оператора $M$, оператора растяжения $D_{a}$ или оператора $P^{a}$ соответственно. Обратно, композиция с $L$ обращает $M-, D_{a}$ - или $P^{a}$-инвариантный непрерывный функиионал на $L_{\infty}\left(\mathbb{R}_{+}^{*}\right)$ в $H$-, $T_{a}$ - или $D_{a}$-инвариантный непрерывный функционал на $L_{\infty}(\mathbb{R})$.

Мы обозначим через $C_{0}(\mathbb{R})$ (соответственно $\left.C_{0}\left(\mathbb{R}_{+}^{*}\right)\right)$ пространство непрерывных функций на $\mathbb{R}$ (соответственно $\mathbb{R}_{+}^{*}$ ), обращающихся в нуль на бесконечности.

Доказательство следующей теоремы аналогично доказательству теоремы 4.3.

Теорема 4.7 [29]. Существует состояние $\widetilde{\omega}$ на $L_{\infty}(\mathbb{R})$, удовлетворяющее следующим условиям:

(1) $\widetilde{\omega}\left(C_{0}(\mathbb{R})\right) \equiv 0$;

(2) если $f$ - вещественнозначная функиия из $L_{\infty}(\mathbb{R})$, то

$$
\text { ess } \liminf _{t \rightarrow \infty} f(t) \leqslant \widetilde{\omega}(f) \leqslant \text { ess } \limsup _{t \rightarrow \infty} f(t)
$$

(3) если существенный носитель функции $f$ компактен, то $\widetilde{\omega}(f)=0$;

(4) для любых $a>0$ и $с \in \mathbb{R}$

$$
\widetilde{\omega}=\widetilde{\omega} \circ T_{c}=\widetilde{\omega} \circ D_{a}=\widetilde{\omega} \circ H
$$

Применяя теорему 4.7 и предложение 4.6, мы получаем следующее утверждение.

СЛЕДСТВИЕ 4.8. Существует состояние $\omega$ на $L_{\infty}\left(\mathbb{R}_{+}^{*}\right)$, удовлетворяющее следующим условиям:

(1) $\omega\left(C_{0}\left(\mathbb{R}_{+}^{*}\right)\right) \equiv 0$

(2) если $f$ - вещественнозначная функция из $L_{\infty}\left(\mathbb{R}_{+}^{*}\right)$, то

$$
\text { ess } \liminf _{t \rightarrow \infty} f(t) \leqslant \omega(f) \leqslant \text { ess } \limsup _{t \rightarrow \infty} f(t)
$$

(3) если существенный носитель функции $f$ компактен, то $\omega(f)=0$; 
(4) для любых $a, c>0$

$$
\omega=\omega \circ D_{c}=\omega \circ P^{a}=\omega \circ M .
$$

Результаты, приведенные в теореме 4.7 и следствии 4.8, позволяют развить альтернативный подход к теории следов Диксмье [1] и Конна-Диксмье [7]. В то время как первоначальный подход Диксмье основан на использовании функционалов, инвариантных при растяжениях, мы заменяем последние банаховыми пределами (т.е. функционалами, инвариантными при сдвигах) и используем хорошо развитую теорию почти сходящихся последовательностей.

Мы введем следующие обозначения:

$$
\begin{aligned}
& B L(\mathbb{R})=\left\{\text { все состояния } \widetilde{\omega} \text { на } L_{\infty}(\mathbb{R}), \text { удовлетворяющие условиям }(1)-(3)\right. \\
& \text { теоремы } \left.4.7 \text {, такие, что } \widetilde{\omega} \circ T_{c}=\widetilde{\omega} \text { для любого } c \in \mathbb{R}\right\} \text {, } \\
& D\left(\mathbb{R}_{+}^{*}\right)=\left\{\text { все состояния } \omega \text { на } L_{\infty}\left(\mathbb{R}_{+}^{*}\right),\right. \text { удовлетворяющие условиям (1)-(3) } \\
& \text { следствия } \left.4.8 \text {, такие, что } \omega \circ D_{c}=\omega \text { для любого } c \in \mathbb{R}_{+}^{*}\right\} \text {, } \\
& B L\left(\mathbb{R}_{+}\right)=\left\{\text {все состояния } \widetilde{\omega} \text { на } L_{\infty}\left(\mathbb{R}_{+}\right),\right. \text {удовлетворяющие условиям (1)-(3) } \\
& \text { следствия } \left.4.8 \text {, такие, что } \widetilde{\omega} \circ T_{c}=\widetilde{\omega} \text { для любого } c \in \mathbb{R}_{+}\right\} \text {. }
\end{aligned}
$$

Следующее простое замечание в дальнейшем играет важную роль. Если $\omega \in D\left(\mathbb{R}_{+}^{*}\right)$, то $\mathbb{L}:=\omega \circ L$ принадлежит множеству $B L(\mathbb{R})$. Если $\mathbb{L} \in B L\left(\mathbb{R}_{+}\right)$, то $\widetilde{\omega}:=\mathbb{L} \circ L^{-1}$ принадлежит множеству $D\left(\mathbb{R}_{+}^{*}\right)$. Наконец, заметим, что изоморфизм $L: L_{\infty}(\mathbb{R}) \rightarrow L_{\infty}\left(\mathbb{R}_{+}^{*}\right)$ отображает пространство $C_{b}(\mathbb{R})$ всех ограниченных непрерывных функций на $\mathbb{R}$ на пространство $C_{b}\left(\mathbb{R}_{+}\right)$всех ограниченных непрерывных функций на $\mathbb{R}_{+}$. Таким образом, можно переформулировать все результаты из предложений 4.5, 4.6, теоремы 4.7 и следствия 4.8 для пространств непрерывных ограниченных функций на $\mathbb{R}$ и $\mathbb{R}_{+}$.

\section{5. Конкретные конструкции сингулярных симметричных функционалов}

5.1. Следы Диксмье. Если $\omega$ - состояние на $\ell_{\infty}\left(\right.$ соответственно на $L_{\infty}(\mathbb{R})$ или $L_{\infty}\left(\mathbb{R}_{+}^{*}\right)$ ), то мы часто будем обозначать его значение на элементе $\left\{x_{i}\right\}_{i=1}^{\infty}$ (соответственно $f \in L_{\infty}(\mathbb{R})$ или $L_{\infty}\left(\mathbb{R}_{+}^{*}\right)$ ) через $\omega$ - $\lim _{i \rightarrow \infty} x_{i}$ (соответственно $\left.\omega-\lim _{t \rightarrow \infty} f(t)\right)$. Напомним, что теоремы $4.3,4.7$ и следствие 4.8 гарантируют существование инвариантных при сдвиге и/или растяжениях состояний на $\ell_{\infty}$, $L_{\infty}(\mathbb{R}), L_{\infty}\left(\mathbb{R}_{+}^{*}\right)$. Для простоты мы объясним конструкцию следов Диксмье для идеала компактных операторов $\left(\mathscr{L}^{(1, \infty)}(\mathscr{H}),\|\cdot\|_{(1, \infty)}\right)$, определенного в п. 2.1.

ОПРЕДЕЛЕНИЕ 5.1. Пусть $\omega-D_{2}$-инвариантное состояние на $\ell_{\infty}$. След Диксмъе элемента $T \in \mathscr{L}_{+}^{(1, \infty)}(\mathscr{H})-$ это число

$$
\tau_{\omega}(T):=\omega_{N \rightarrow \infty} \frac{1}{\log (1+N)} \sum_{n=1}^{N} \mu_{n}(T) .
$$

ЗАмЕчАниЕ. В приведенном ниже доказательстве мы преднамеренно выбрали $\omega$ удовлетворяющим только условию инвариантности при растяжениях, хотя Диксмье первоначально накладывал на $\omega$ условие инвариантности при растяжениях и сдвигах. Мы обсудим различия ниже. 
ПРеДЛОЖеНИЕ 5.2. $\tau_{\omega}(S+T)=\tau_{\omega}(S)+\tau_{\omega}(T)$ для всех $S, T \in \mathscr{L}_{+}^{(1, \infty)}(\mathscr{H})$. ДокАЗАТЕльство. Положим, для краткости,

$$
\sigma_{N}(X):=\sum_{n=1}^{N} \mu_{n}(X), \quad X \in \mathscr{L}^{(1, \infty)}(\mathscr{H})
$$

и заметим, что

$$
\sigma_{N}(X)=\sup \{\operatorname{Tr}(X P): P=P(\mathscr{H})-\text { проектор и } \operatorname{dim} P(\mathscr{H})=N\}
$$

(см., например, [49], [47]). Для данного $\varepsilon>0$ пусть проекторы $P_{1}$ и $P_{2}$ удовлетворяют условиям $\operatorname{dim} P_{1}(\mathscr{H})=\operatorname{dim} P_{2}(\mathscr{H})=N$ и $\operatorname{Tr}\left(S P_{1}\right)>\sigma_{N}(S)-\varepsilon$, $\operatorname{Tr}\left(T P_{2}\right)>\sigma_{N}(T)-\varepsilon$. Полагая $P:=P_{1} \vee P_{2}$, имеем

$$
\operatorname{Tr}((S+T) P)=\operatorname{Tr}(S P)+\operatorname{Tr}(T P) \geqslant \operatorname{Tr}\left(S P_{1}\right)+\operatorname{Tr}\left(T P_{2}\right)>\sigma_{N}(S)+\sigma_{N}(T)-2 \varepsilon .
$$

Поскольку $\operatorname{dim} P(\mathscr{H}) \leqslant 2 N$ и $\varepsilon-$ произвольное положительное число, мы имеем

$$
\sigma_{2 N}(S+T) \geqslant \sigma_{N}(S)+\sigma_{N}(T), \quad N \geqslant 1
$$

Полагая, для краткости,

$\alpha_{N}=\frac{1}{\log (1+t)} N \sigma_{N}(S), \quad \beta_{N}=\frac{1}{\log (1+t)} N \sigma_{N}(T), \quad \gamma_{N}=\frac{1}{\log (1+t)} N \sigma_{N}(S+T)$,

мы перепишем предыдущее неравенство в виде

$$
\frac{\log (1+2 N)}{\log (1+N)} \gamma_{2 N} \geqslant \alpha_{N}+\beta_{N}, \quad N \geqslant 1 .
$$

Предположим временно, что следующее равенство уже установлено:

$$
\underset{N \rightarrow \infty}{\omega} \lim _{2 N}=\underset{N \rightarrow \infty}{\omega} \gamma_{N}
$$

Замечая, что $\left\{\gamma_{2 N}\right\}_{N \geqslant 1} \in \ell_{\infty}$ и поэтому $\frac{\log (1+2 N)}{\log (1+N)} \gamma_{2 N}-\gamma_{2 N} \rightarrow 0$, мы получаем из предыдущего неравенства, что

$$
\tau_{\omega}(S+T)=\underset{N \rightarrow \infty}{\omega} \lim _{N} \geqslant \underset{N \rightarrow \infty}{\omega} \lim _{N} \alpha_{N}+\underset{N \rightarrow \infty}{\omega-\lim } \beta_{N}=\tau_{\omega}(S)+\tau_{\omega}(T) .
$$

Поскольку обратное неравенство $\tau_{\omega}(S+T) \leqslant \tau_{\omega}(S)+\tau_{\omega}(T)$ немедленно следует из хорошо известного неравенства $\sigma_{N}(S+T) \leqslant \sigma_{N}(S)+\sigma_{N}(T)$ (см. [49], [47]), доказательство завершено. Остается обосновать равенство (8).

Заметим, что из $D_{2}$-инвариантности $\omega$ немедленно следует, что

$$
\omega\left(\left\{\gamma_{2 N}\right\}_{N=1}^{\infty}\right)=\omega\left(D_{2}\left\{\gamma_{2 N}\right\}_{N=1}^{\infty}\right)=\omega\left(\left\{\gamma_{2}, \gamma_{2}, \gamma_{4}, \gamma_{4}, \gamma_{6}, \gamma_{6}, \ldots\right\}\right)
$$

и поэтому для того, чтобы доказать (8), достаточно проверить, что $D_{2}\left\{\gamma_{2 N}\right\}_{N=1}^{\infty}-\left\{\gamma_{N}\right\}_{N=1}^{\infty}=\left\{\gamma_{2}, \gamma_{2}, \gamma_{4}, \gamma_{4}, \gamma_{6}, \gamma_{6}, \ldots\right\}-\left\{\gamma_{1}, \gamma_{2}, \gamma_{3}, \gamma_{4}, \gamma_{5}, \ldots\right\} \in c_{0}$ 
или, эквивалентно, что $\gamma_{2 N}-\gamma_{2 N-1} \rightarrow 0$ при $N \rightarrow \infty$. Для $N \geqslant 2$ мы имеем

$$
\gamma_{2 N}-\gamma_{2 N-1}=\left(\frac{1}{\log 2 N}-\frac{1}{\log (2 N-1)}\right) \sigma_{2 N-1}(T+S)+\frac{1}{\log 2 N} \cdot \mu_{2 N}(T+S) .
$$

Очевидно, что второе слагаемое в правой части стремится к 0 при $N \rightarrow \infty$. Замечая, что условие $T+S \in \mathscr{L}^{(1, \infty)}(\mathscr{H})$ гарантирует нам, что

$$
\sigma_{2 N-1}(T+S)=O(\log (2 N-1)) \quad \text { и } \quad \frac{1}{\log 2 N}-\frac{1}{\log (2 N-1)}=o\left(\frac{1}{\log (2 N-1)}\right),
$$

мы видим, что первое слагаемое также стремится к 0 при $N \rightarrow \infty$. Предложение 5.2 доказано.

ЗАмечание 5.3. Рассмотрим изометрическое вложение $i: \ell_{\infty} \rightarrow L_{\infty}[0, \infty)$, задаваемое формулой $\left\{x_{j}\right\}_{j=1}^{\infty} \stackrel{i}{\mapsto} \sum_{j=1}^{\infty} x_{j} \chi_{[j-1, j)}$, где $\chi_{[j-1, j)}$ - характеристическая функция интервала $[j-1, j)$. Отметим, что если $i\left(\left\{\gamma_{N}\right\}_{N \geqslant 1}\right)=f$, то $i\left(\left\{\gamma_{2 N}\right\}_{N \geqslant 1}\right)=f \circ D_{\frac{1}{2}}$. Поэтому доказательство приведенного выше равенства (8) было бы тривиально, если бы $\omega$ было $D_{\frac{1}{2}}$-инвариантным состоянием на $L_{\infty}[0, \infty)$.

ОПРЕДЕЛЕНИЕ 5.4. Для самосопряженного оператора $T \in \mathscr{L}^{(1, \infty)}(\mathscr{H})$ его след Диксмье есть $\tau_{\omega}(T):=\tau_{\omega}\left(T_{+}\right)-\tau_{\omega}\left(T_{-}\right)$, и для произвольного оператора $T \in \mathscr{L}^{(1, \infty)}(\mathscr{H})$ след Диксмье есть $\tau_{\omega}(T):=\tau_{\omega}(\operatorname{Re} T)+i \tau_{\omega}(\operatorname{Im} T)$.

ПреДЛОЖеНИЕ 5.5. След Диксмъе $\tau_{\omega}$ есть (сингулярный) симметричный функционал на $\mathscr{L}^{(1, \infty)}(\mathscr{H})$ такой, что $\tau_{\omega}(S T)=\tau_{\omega}(T S)$ для любых операторов $T \in \mathscr{L}^{(1, \infty)}(\mathscr{H}), S \in B(\mathscr{H})$.

Доказательство. Неравенство $\left|\tau_{\omega}(x)\right| \leqslant\|x\|_{(1, \infty)}, x \in \mathscr{L}^{(1, \infty)}(\mathscr{H})$, и затем тот факт, что $\tau_{\omega}$ есть симметричный функционал, следуют немедленно из определения 5.1 и следствия 4.8. Поскольку каждый оператор $S \in \mathscr{L}(\mathscr{H})$ есть линейная комбинация четырех унитарных операторов [59; VI.6], достаточно доказать равенство $\tau_{\omega}(U T)=\tau_{\omega}(T U)$ для унитарного оператора $U$. Поскольку каждый оператор из $\mathscr{L}(\mathscr{H})$ есть линейная комбинация положительных операторов, достаточно доказать последнее равенство для положительных операторов $T$. А для таких $T$ последнее равенство немедленно следует из того, что $\mu_{n}\left(U T U^{*}\right)=\mu_{n}(U T)=\mu_{n}(T U)=\mu_{n}(T)$ для всех $n \geqslant 1$. Предложение 5.5 доказано.

Определения 5.1 и 5.4 можно распространить на пространства Марцинкевича $\mathscr{L}^{(1, \infty)}(\mathscr{N}, \tau)$ и далее на пространства Марцинкевича $M(\psi)(\mathscr{N}, \tau)$, где $\psi \in \Omega$ удовлетворяет условию (5). Точнее, зафиксируем произвольное состояние $\omega$ на $L_{\infty}\left(\mathbb{R}_{+}^{*}\right)$, удовлетворяющее условиям (1)-(3) следствия 4.8 и являющееся $D_{\frac{1}{2}}$-инвариантным. Полагая

$$
\tau_{\omega}(x):=\underset{t \rightarrow \infty}{\omega} \lim a(x, t), \quad 0 \leqslant x \in M(\psi)(\mathscr{N}, \tau),
$$

и слегка модифицируя рассуждение (см. детали в [31; с. 51]), приведенное при доказательстве предложений 5.2 и 5.5, мы получим аддитивный однородный функционал на $M(\psi)(\mathscr{N}, \tau)_{+}$, который продолжается до симметричного функционала на $M(\psi)(\mathscr{N}, \tau)$ по линейности. 
В дальнейшем мы будем называть любой функционал $\tau_{\omega}$, определенный в $(9)$, где $\omega \in D\left(\mathbb{R}_{+}^{*}\right)$, следом Диксмье.

Наконец, заметим, что двойственность между инвариантными относительно растяжений функционалами на $L_{\infty}\left(\mathbb{R}_{+}^{*}\right)$ и инвариантными относительно сдвигов функционалами на $L_{\infty}(\mathbb{R})$ позволяет дать альтернативное определение следов Диксмье. Для простоты мы рассмотрим это определение только для пространства $\mathscr{L}^{(1, \infty)}(\mathscr{N}, \tau)$, где $(\mathscr{N}, \tau)$ - произвольная полуконечная алгебра фон Неймана.

Пусть $\mathbb{L}$ (соответственно $\mathbf{L})$ принадлежит классу $B L\left(\mathbb{R}_{+}\right)$(соответственно $B L(\mathbb{N}))$. Положим

$$
\begin{array}{ll}
F_{\mathbb{L}}(T):=\mathbb{L}_{t \rightarrow \infty}-\lim \frac{1}{\log \left(1+e^{t}\right)} \int_{0}^{e^{t}} \mu_{s}(T) d s, & \\
F_{\mathbf{L}}(T):={\underset{L}{L}-\lim }_{N} \frac{1}{\log \left(1+e^{N}\right)} \sum_{n=1}^{\left[e^{N}\right]} \mu_{n}(T), &
\end{array}
$$

где $\left[e^{N}\right]$ - целая часть числа $e^{N}$.

Теорема 5.6 [29], [40], [41], [60]. Для любой полуконечной алгебры фон Неймана $(\mathscr{N}, \tau)$ и произвольных состояний $\mathbb{L} \in B L\left(\mathbb{R}_{+}\right)$u $\mathbf{L} \in B L(\mathbb{N})$ функциональ $F_{\mathbb{L}}$ и $F_{\mathbf{L}}$ являются симметричными функиионалами на $\mathscr{L}^{(1, \infty)}(\mathscr{N}, \tau)$.

Следующая теорема показывает, что класс следов Диксмье на $\mathscr{L}^{(1, \infty)}(\mathscr{N}, \tau)$ совпадает с множествами функционалов $\left\{F_{\mathbf{L}}: \mathbf{L} \in B L(\mathbb{N})\right\}$ и $\left\{F_{\mathbb{L}}: \mathbb{L} \in B L\left(\mathbb{R}_{+}\right)\right\}$. Доказательство первого из приведенных ниже равенств следует из замечаний, сделанных в конце предыдущего раздела.

Теорема 5.7 [60; теоремы 2.3, 6.2]. Для любой полуконечной алгебры фон Неймана $(\mathscr{N}, \tau)$ мы имеем

$$
\left\{\tau_{\omega}: \omega \in D\left(\mathbb{R}_{+}^{*}\right)\right\}=\left\{F_{\mathbb{L}}: \mathbb{L} \in B L\left(\mathbb{R}_{+}\right)\right\}=\left\{F_{\mathbf{L}}: \mathbf{L} \in B L(\mathbb{N})\right\}
$$

Подробное изучение класса вогнутых функций $\psi \in \Omega$, для которых справедливы аналоги теорем 5.6 и 5.7 для аналогично определяемых классов симметричных функционалов на пространствах Марцинкевича $M(\psi)(\mathscr{N}, \tau)$, содержится в работах [40], [41], [60].

5.2. Следы Конна-Диксмье. В предыдущем пункте мы показали, что для каждого состояния $\omega \in D\left(\mathbb{R}_{+}^{*}\right)$ (соответственно $\mathbb{L} \in B L\left(\mathbb{R}_{+}\right), B L(\mathbb{R}), \mathbf{L} \in$ $B L(\mathbb{N})$ ) имеется ассоциированный след Диксмье $\tau_{\omega}$ (соответственно симметричный функционал $\left.F_{\mathbb{L}}, F_{\mathbf{L}}\right)$. Можно выделить различные подмножества в множествах состояний $D\left(\mathbb{R}_{+}^{*}\right), B L\left(\mathbb{R}_{+}\right), B L(\mathbb{R}), B L(\mathbb{N})$ и связать их с соответствующими подмножествами следов. Например, рассмотрим множества всех $H$-инвариантных (соответственно $M$-инвариантных) состояний на $L_{\infty}(\mathbb{R})$ (или на $\left.\ell_{\infty}\right)$ (соответственно на $\left.L_{\infty}\left(\mathbb{R}_{+}^{*}\right)\right)$. Легко видеть, что

$$
\begin{gathered}
\left\{\omega: \omega-H \text {-инвариантное состояние на } L_{\infty}(\mathbb{R})\left(\text { соотв. на } \ell_{\infty}\right)\right\} \\
\varsubsetneqq\{\omega: \omega \in B L(\mathbb{R})(\text { соотв. } B L(\mathbb{N}))\}
\end{gathered}
$$


$\left\{\omega: \omega-M\right.$-инвариантное состояние на $\left.L_{\infty}\left(\mathbb{R}_{+}^{*}\right)\right\} \varsubsetneqq\left\{\omega: \omega \in D\left(\mathbb{R}_{+}^{*}\right)\right\}$.

В самом деле, предположим, что $0 \leqslant \omega \in \ell_{\infty}^{*}$ удовлетворяет условиям $\omega(\mathbf{1})=1$ и $\omega(H x)=\omega(x)$ для любого $x \in \ell_{\infty}$. Чтобы доказать, что $\omega(T x)=\omega(x), x \in \ell_{\infty}$, достаточно показать, что $\omega(H T x)=\omega(H x), x \in \ell_{\infty}$. Однако непосредственное вычисление дает

$$
(H T x)_{N}-(H x)_{N}=\frac{x_{2}+\cdots+x_{N+1}}{N}-\frac{x_{1}+\cdots+x_{N}}{N}=\frac{x_{N+1}-x_{1}}{N} \rightarrow 0
$$

при $N \rightarrow \infty$. Аналогично, можно показать, что для любых $x \in L_{\infty}(\mathbb{R})$ и $b \in \mathbb{R}$ мы имеем

$$
\lim _{t \rightarrow \infty}\left(\left(H T_{b} x\right)(t)-(H x)(t)\right)=0
$$

что устанавливает соотношение (10), и включение (11) следует из (10) ввиду следствия 4.8. А. Конн в [7] предложил работать с множеством состояний на $L_{\infty}\left(\mathbb{R}_{+}^{*}\right)$, которое больше, чем множество, стоящее в левой части соотношения (11). Именно, рассмотрим следующий класс состояний на $L_{\infty}\left(\mathbb{R}_{+}^{*}\right)$ :

$C D\left(\mathbb{R}_{+}^{*}\right):=\left\{\widetilde{\omega}=\gamma \circ M: \gamma-\right.$ произвольное сингулярное состояние на $\left.C_{b}[0, \infty)\right\}$.

Все еще легко проверить, что $C D\left(\mathbb{R}_{+}^{*}\right) \varsubsetneqq D\left(\mathbb{R}_{+}^{*}\right)$, и затем из предложения 4.5 вывести справедливость собственного включения $C B L\left(\mathbb{R}_{+}\right) \varsubsetneqq B L\left(\mathbb{R}_{+}\right)$, где

$C B L\left(\mathbb{R}_{+}\right):=\left\{\mathbb{L}=\gamma \circ H: \gamma-\right.$ произвольное сингулярное состояние на $\left.C_{b}\left(\mathbb{R}_{+}\right)\right\}$.

Мы будем говорить о подклассе следов Диксмье

$$
\left\{\tau_{\omega}: \omega \in C D\left(\mathbb{R}_{+}^{*}\right)\right\}
$$

как о классе следов Конна-Диксмье. Следующая теорема показывает, что аналог теоремы 5.7 справедлив также для класса следов Конна-Диксмье.

Теорема 5.8 [60; теоремы 5.6, 6.2]. Для каждой полуконечной алгебры фон Неймана $(\mathscr{N}, \tau)$ мы имеем

$$
\left\{\tau_{\omega}: \omega \in C D\left(\mathbb{R}_{+}^{*}\right)\right\}=\left\{F_{\mathbb{L}}: \mathbb{L} \in C B L\left(\mathbb{R}_{+}\right)\right\} .
$$

В заключение этого пункта заметим, что имеется другой естественный подкласс следов Диксмье, который ассоциирован со следующим подмножеством состояний на $L_{\infty}\left(\mathbb{R}_{+}^{*}\right)$, появляющимся в следствии 4.8 :

$$
\begin{gathered}
\left\{\omega \in L_{\infty}\left(\mathbb{R}_{+}^{*}\right)^{*}: \omega-M \text {-инвариантное и } P^{a}\right. \text {-инвариантное } \\
\text { состояние на } \left.L_{\infty}\left(\mathbb{R}_{+}^{*}\right), a>0\right\}
\end{gathered}
$$

или, эквивалентно, с множеством

$$
\begin{aligned}
\left\{\mathbb{L} \in L_{\infty}(\mathbb{R})^{*}\right. & : \mathbb{L}-H \text {-инвариантное и } D_{a} \text {-инвариантное } \\
& \text { состояние на } \left.L_{\infty}(\mathbb{R}), a>0\right\} .
\end{aligned}
$$

В дальнейшем мы будем ссылаться на класс следов Диксмье, ассоциированный с последним множеством, как на класс максимально инвариантных следов Диксмье. Очевидно, что последний класс содержится в классе следов КоннаДиксмье. Максимально инвариантные следы Диксмье используются в разделах 10 и 11. 
5.3. Перестановочно-инвариантные функционалы и сингулярные следы. Изучавшийся в предыдущих пунктах класс следов Диксмье (и его подклассы) является специальным классом общего класса (сингулярных) симметричных функционалов на (вполне симметричном) операторном пространстве $\mathscr{L}^{(1, \infty)}(\mathscr{N}, \tau)$ (или на операторном идеале $\left.\mathscr{L}^{(1, \infty)}(\mathscr{H})\right)$. Последний класс, в свою очередь, является подклассом класса перестановочно-инвариантных функционалов на $\mathscr{L}^{(1, \infty)}(\mathscr{N}, \tau)$. В случае операторного идеала $\mathscr{L}^{(1, \infty)}(\mathscr{H})$ последний класс можно рассматривать как множество всех (сингулярных) следов на $\mathscr{L}(\mathscr{H})$, которые принимают конечные значения на элементах из $\mathscr{L}^{(1, \infty)}(\mathscr{H})$. Литература, посвященная общим сингулярным следам на $\mathscr{L}(\mathscr{H})$, огромна. Мы ограничим наш список работ из этой области статьями [33], [34], [18]-[20], [61], [62]. Во многих случаях теория сингулярных следов параллельна теории симметричных функционалов. Например, результаты работ [18], [62] (соответственно [33]), касающиеся необходимых и достаточных условий существования для заданного положительного компактного оператора $T$ (соответственно положительного $\tau$-измеримого оператора $\left.T \in L_{1}(\mathscr{N}, \tau)+\mathscr{N}\right)$ сингулярного следа, принимающего конечное ненулевое значение на $T$, на самом деле очень близки к результату, сформулированному в теореме 2.2. Следует заметить, что результаты работы [33] установлены для общих $\tau$-измеримых операторов, а не только для операторов из $L_{1}(\mathscr{N}, \tau)+\mathscr{N}$, тем не менее, не все приведенные в этой работе результаты, рассматривающие этот общий случай, снабжены надежным доказательством (см., например, [33; предложение 3.3]).

Наконец, укажем на важные связи между теорией общих сингулярных следов и изучением структуры пространств коммутаторов для операторов, действующих на гильбертовом пространстве (см. комментарии, сделанные в конце раздела 3). Последние из упомянутых выше исследований связаны с теорией циклических гомологий и алгебраической $K$-теорией операторных идеалов и находятся вне компетенции данного обзора. По поводу более подробной информации мы рекомендуем работы [32], [54], [56], [55], [57].

\section{6. Класс измеримых элементов}

В этом разделе мы даем краткий обзор понятия измеримых операторов, введенного А. Конном [7].

ОПреДЕЛЕНИЕ 6.1 . Оператор $T \in \mathscr{L}^{(1, \infty)}(\mathscr{H})$ называется измеримым (по Диксмъе $)$, если $\tau_{\omega}(T)$ не зависит от выбора $\omega \in D\left(\mathbb{R}_{+}^{*}\right)$.

ОПределениЕ 6.2 [7]. Оператор $T \in \mathscr{L}^{(1, \infty)}(\mathscr{H})$ называется измеримым (по Конну-Диксмъе), если $\tau_{\omega}(T)$ не зависит от выбора $\omega \in C D\left(\mathbb{R}_{+}^{*}\right)$.

ЗАмечАниЕ 6.3. (i) Очевидно, что определенные выше множества измеримых операторов являются линейными пространствами, которые, фактически, являются замкнутыми подпространствами в $\mathscr{L}^{(1, \infty)}(\mathscr{H})$. Однако эти подпространства не являются порядковыми идеалами, иными словами, из того, что самосопряженный оператор $A$ измерим, не обязательно следует, что $A_{+}$и $A_{-}-$ измеримые операторы. Приведем пример. Возьмем положительный неизмеримый диагональный оператор $A=\operatorname{diag}\left\{a_{1}, a_{2}, a_{3}, \ldots\right\}$ из $\mathscr{L}^{(1, \infty)}(\mathscr{H})$. Определим диагональный оператор $B$ по формуле $B=\operatorname{diag}\left\{a_{1},-a_{1}, a_{2},-a_{2}, \ldots\right\}$. 
Очевидно, что $B$ измерим, более того, $\tau_{\omega}(B)=0$ для любого $\omega$. Однако положительная и отрицательная части оператора $B$ не измеримы.

(ii) Определения операторов, измеримых по Диксмье и по Конну-Диксмье, естественным образом распространяются [60] на пространства Марцинкевича $\mathscr{L}^{(1, \infty)}(\mathscr{N}, \tau)$, где $(\mathscr{N}, \tau)$ - произвольная полуконечная алгебра фон Неймана, и далее на операторные пространства Марцинкевича $M(\psi)(\mathscr{N}, \tau)$ для всех $\psi$, удовлетворяющих условию (3).

Очевидно, что каждый измеримый по Диксмье оператор также измерим по Конну-Диксмье. Наша цель в этом разделе состоит в том, чтобы описать классы положительных измеримых по Конну-Диксмье операторов и положительных измеримых по Диксмье операторов и показать, что эти два класса на самом деле совпадают. Для этого нам необходимы два вспомогательных результата.

ТЕорема 6.4 [63; раздел 6.8]. Пусть b(t) - такая положительная кусочно дифферениируемая функиия, что $t b^{\prime}(t)>-H$ при некотором $H>0$ и при всех $t>C$, где $C$ - некоторая постоянная. Тогда

$$
\lim _{t \rightarrow \infty} \frac{1}{t} \int_{0}^{t} b(s) d s=A \quad \text { при некотором } A \geqslant 0 \Leftrightarrow \lim _{t \rightarrow \infty} b(t)=A .
$$

Имитируя определение Лоренца почти сходящихся последовательностей, скажем, что положительная функция $f \in C_{b}[0, \infty)$ является почти сходящейся, если все состояния из $B L\left(\mathbb{R}_{+}\right)$принимают одно и то же значение на этой функции.

Теорема 6.5 [60; теорема 3.3]. Если функиия $f \in C_{b}[0, \infty)$ почти сходится $\kappa$ числу $A$, то предел

$$
\lim _{t \rightarrow \infty} \frac{1}{t} \int_{0}^{t} f(s) d s
$$

существует и равен $A$.

ДокАЗАТЕЛЬСтво. Предположим, что результат не верен. Тогда существует такая постоянная $c \neq A$, что $(H f)\left(t_{n}\right) \rightarrow c$ для некоторой последовательности $t_{n} \uparrow \infty$. Возьмем единичный шар $B$ пространства $C_{b}([0, \infty))^{*}$ и рассмотрим последовательность функционалов $\sigma_{t_{n}}(x)=x\left(t_{n}\right), n \geqslant 1$, из $B$. Поскольку $B$ *-слабо компактен, эта последовательность имеет предельную точку $V \in B$. Легко видеть, что $V \geqslant 0, V(1)=1, V(p)=\lim _{n \rightarrow \infty} p\left(t_{n}\right)=0$ для любого $p \in C_{0}[0, \infty)$ и также что $V(H(f))=\lim _{n \rightarrow \infty} H(f)\left(t_{n}\right)=c$. Определим функционал $L$ на $L_{\infty}\left(\mathbb{R}_{+}\right)$, полагая $L(x):=V(H(x))$. Легко проверить, что $L$ является состоянием из $B L\left(\mathbb{R}_{+}\right)$и $V(f)=c \neq A$. Таким образом, предположение о том, что результат не верен, ложно.

Следующая теорема является основным результатом этого раздела.

ТеОрема 6.6 [60]. Положительный оператор $T$ из $\mathscr{L}^{(1, \infty)}(\mathscr{N}, \tau)$ измерим по Диксмъе тогда и только тогда, когда существует предел

$$
\lim _{t \rightarrow \infty} \frac{1}{\log (1+t)} \int_{0}^{t} \mu_{s}(T) d s
$$


ДокАЗАТЕЛьство. Часть “тогда" утверждения тривиальна. Зафиксируем такой оператор $T \in \mathscr{L}_{+}^{(1, \infty)}(\mathscr{N}, \tau)$, что для $g(t):=\frac{1}{\log (1+t)} \int_{0}^{t} \mu_{s}(T) d s$ мы имеем $\tau_{\omega}(T)=\omega-\lim _{t \rightarrow \infty} g(t)=A \geqslant 0$ для любого $\omega \in C D\left(\mathbb{R}_{+}^{*}\right)$. Из приведенных в п. 5.2 замечаний следует, что для любого $\mathbb{L} \in B L\left(\mathbb{R}_{+}\right)$мы имеем $\operatorname{Tr}_{\mathbb{L}}(T):=\mathbb{L}-\lim _{\lambda \rightarrow \infty} g\left(e^{\lambda}\right)=A$, и поэтому по теореме 6.5 мы получим

$$
\lim _{u \rightarrow \infty} \frac{1}{u} \int_{0}^{u}\left(\frac{1}{\log \left(1+e^{\lambda}\right)} \int_{0}^{e^{\lambda}} \mu_{s}(T) d s\right) d \lambda=A .
$$

Полагая

мы имеем

$$
b(\lambda):=\frac{1}{\log \left(1+e^{\lambda}\right)} \int_{0}^{e^{\lambda}} \mu_{s}(T) d s, \quad \lambda>0
$$

$$
\begin{aligned}
\lambda b^{\prime}(\lambda) & \geqslant \lambda \frac{d}{d \lambda}\left(\frac{1}{\log \left(1+e^{\lambda}\right)}\right) \int_{0}^{e^{\lambda}} \mu_{s}(T) d s=-\frac{\lambda e^{\lambda}}{\left(1+e^{\lambda}\right) \log ^{2}\left(1+e^{\lambda}\right)} \int_{0}^{e^{\lambda}} \mu_{s}(T) d s \\
& \geqslant-\frac{\lambda}{\log \left(1+e^{\lambda}\right)} \cdot \frac{1}{\log \left(1+e^{\lambda}\right)} \int_{0}^{e^{\lambda}} \mu_{s}(T) d s \geqslant-\|T\|_{(1, \infty)} .
\end{aligned}
$$

Применяя теорему 6.4, мы приходим к заключению, что $\lim _{\lambda \rightarrow \infty} b(\lambda)=A$, и поэтому $\lim _{t \rightarrow \infty} g(t)=A$. Теорема 6.6 доказана.

Теперь мы покажем, что рассуждение, аналогичное рассуждению в приведенном выше доказательстве, дает более сильный результат.

Теорема 6.7 [60]. Положительный оператор $T$ из $\mathscr{L}^{(1, \infty)}(\mathscr{N}, \tau)$ измерим по Конну-Диксмье тогда и только тогда, когда существует предел

$$
\lim _{t \rightarrow \infty} \frac{1}{\log (1+t)} \int_{0}^{t} \mu_{s}(T) d s .
$$

ДокАЗАтЕльство. Нам необходимо только доказать часть "только тогда". Мы будем использовать обозначения $g(\cdot)$ и $b(\cdot)$, введенные в ходе доказательства теоремы 6.6. Предположим, что оператор $T \in \mathscr{L}_{+}^{(1, \infty)}(\mathscr{N}, \tau)$ удовлетворяет равенству $A=\gamma \circ M(g)=\gamma \circ L H L^{-1}(g)$ для любого состояния $\gamma \in C_{b}^{*}[0, \infty)$, обращающегося в нуль на $C_{0}(0, \infty)$.

Заметим, что если $\gamma$ - инвариантное при растяжениях состояние, то $\gamma \circ L-$ инвариантное при сдвигах состояние. Это замечание (и тот факт, что $L: L_{\infty}(\mathbb{R}) \rightarrow L_{\infty}\left(\mathbb{R}_{+}^{*}\right)$ - изоморфизм) показывает, что функция $H L^{-1}(g)=H b$ почти сходится. Применяя теорему 6.5 к функции $H b$, мы видим, что предел $\lim _{t \rightarrow \infty} H H(b)(t)$ существует. Предположим на время, что мы уже проверили предположение теоремы 6.4 для функции $H b$. Тогда из этой теоремы мы можем заключить, что предел $\lim _{t \rightarrow \infty} H(b)(t)$ также существует, и, повторяя использование этой же самой теоремы (как в доказательстве теоремы 6.6), мы заключаем, что существует предел $\lim _{t \rightarrow \infty} b(t)$ и, следовательно, предел $\lim _{\lambda \rightarrow \infty} g(\lambda)$.

Остается проверить предположение теоремы 6.4 для функции $H b$. Для любого $\lambda \geqslant 1$ мы имеем

$$
\lambda(H b)^{\prime}(\lambda)=\frac{\lambda b(\lambda)-\int_{0}^{\lambda} b(s) d s}{\lambda} \geqslant-\|b\|_{\infty} \geqslant-\|T\|_{(1, \infty)} .
$$


Теорема 6.7 доказана.

СлЕДСТвИЕ 6.8. Множества всех положительных измеримых по Диксмъе операторов и всех положительных измеримых по Конну-Диксмъе операторов совпадают.

Следующие вопросы остаются открытыми:

(i) Совпадают ли пространства измеримых по Диксмье и измеримых по Конну-Диксмье операторов?

(ii) Каково описание множества всех (положительных) операторов, которые измеримы по отношению к множеству максимально инвариантных следов Диксмье?

(iii) Каково описание множества всех (положительных) операторов, которые измеримы по отношению к множеству всех симметричных функционалов?

Можно было бы пытаться поставить вопрос (iii) в большей общности и спросить, можно ли разумно сформулировать понятие операторов, измеримых по отношению к множеству всех перестановочно-инвариантных функционалов. Имеется пример, приведенный в [35] (см. выше конец раздела 2), который дает отрицательный ответ на этот вопрос.

По поводу результатов, распространяющих теоремы 6.6 и 6.7 на пространства Марцинкевича $M(\psi)(\mathscr{N}, \tau)$ с $\psi \in \Omega$, удовлетворяющим условию (4), мы отсылаем читателя к работе [60].

\section{7. Нормирующие свойства функционалов Диксмье и Конна-Диксмье}

У читателя может сложиться впечатление, что следы Диксмье и КоннаДиксмье образуют очень “тощее” подмножество единичной сферы в двойственном пространстве. Такое впечатление ложно, как установлено в приведенных ниже теоремах 7.3 и 7.4. Нам будет необходима следующая теорема Сачестона.

Теорема 7.1 [64]. Для любого $x \in \ell_{\infty}$

$$
\sup _{\mathbf{L} \in B L(\mathbb{N})} \mathbf{L}(x)=\lim _{n \rightarrow \infty}\left(\sup _{m} \frac{1}{n} \sum_{j=1}^{n} x_{m+j}\right) .
$$

Следующее предложение легко следует из его "коммутативного" аналога, который, в свою очередь, можно извлечь из [42].

ПреДЛОЖеНИЕ 7.2. Пусть $T \in \mathscr{L}^{(1, \infty)}(\mathscr{N}, \tau)$. Расстояние от $T$ до подпространства $\mathscr{L}_{0}^{(1, \infty)}(\mathscr{N}, \tau)$ в норме $\|\cdot\|_{(1, \infty)}$ равно

$$
\rho(T):=\limsup _{t \rightarrow \infty} \frac{1}{\log (1+t)} \int_{0}^{t} \mu_{s}(T) d s .
$$

Напомним, что в частном случае $\mathscr{L}^{(1, \infty)}(\mathscr{N}, \tau)=\mathscr{L}^{(1, \infty)}(\mathscr{H})$ пространство $\mathscr{L}_{0}^{(1, \infty)}(\mathscr{H})$ есть замкнутая линейная оболочка в $\mathscr{L}^{(1, \infty)}(\mathscr{H})$ множества всех конечномерных операторов. 
Tеорема 7.3 [60]. Пусть $T \in \mathscr{L}^{(1, \infty)}(\mathscr{N}, \tau)$. Расстояние от $T$ до подпространства $\mathscr{L}_{0}^{(1, \infty)}(\mathscr{N}, \tau)$ в норме $\|\cdot\|_{(1, \infty)}$ равно $\sup \left\{\tau_{\omega}(|T|): \omega \in D\left(\mathbb{R}_{+}^{*}\right)\right\}$.

ДоказАтельство. Достаточно рассмотреть случай $T \geqslant 0$. Прежде всего, заметим, что

$\sup \left\{\tau_{\omega}(T): \omega \in D\left(\mathbb{R}_{+}^{*}\right)\right\}=\sup \left\{\operatorname{Tr}_{\mathbb{L}}(T): \mathbb{L} \in B L\left(\mathbb{R}_{+}\right)\right\}=\sup \left\{\operatorname{Tr}_{\mathbf{L}}(T): \mathbf{L} \in B L(\mathbb{N})\right\}$.

Очевидно, что

$$
q(T):=\sup _{\mathbf{L} \in B L(\mathbb{N})} \operatorname{Tr}_{\mathbf{L}}(T) \leqslant \limsup _{t \rightarrow \infty} \frac{1}{\log (1+t)} \int_{0}^{t} \mu_{s}(T) d s \quad(=\rho(T)) .
$$

Мы должны доказать обратное неравенство $q(T) \geqslant \rho(T)$. По теореме Сачестона 7.1 достаточно доказать, что

$$
\forall \varepsilon>0 \exists N \in \mathbb{N} \forall n \geqslant N \exists m \in \mathbb{N}: \frac{1}{n} \sum_{j=1}^{n} \frac{1}{m+j} \int_{0}^{e^{m+j}} \mu_{s}(T) d s \geqslant \rho(T)-\varepsilon .
$$

Для этого достаточно положить $N=1$ и взять $m$ таким, что

$$
\frac{m}{m+n}>\frac{\rho(T)-\varepsilon}{\rho(T)-\varepsilon / 2} \quad \text { и } \quad \frac{1}{\log \left(1+e^{m}\right)} \int_{0}^{e^{m}} \mu_{s}(T) d s>\rho(T)-\varepsilon / 2 .
$$

Тогда

$$
\begin{gathered}
\frac{1}{n} \sum_{j=1}^{n} \frac{1}{m+j} \int_{0}^{e^{m+j}} \mu_{s}(T) d s \geqslant \frac{1}{n} \sum_{j=1}^{n} \frac{1}{m+n} \int_{0}^{e^{m}} \mu_{s}(T) d s \\
=\frac{m}{m+n} \cdot \frac{1}{m} \int_{0}^{e^{m}} \mu_{s}(T) d s>\rho(T)-\varepsilon .
\end{gathered}
$$

Для того чтобы убедиться, что выбор $m$, удовлетворяющего (12), возможен, определим сначала последовательность $1 \leqslant t_{1}<t_{2}<\cdots \uparrow \infty$ такую, что

$$
\lim _{k \rightarrow \infty} \frac{1}{\log \left(1+t_{k}\right)} \int_{0}^{t_{k}} \mu_{s}(T) d s>\rho(T)-\varepsilon / 4, \quad k \geqslant 1
$$

(это можно сделать благодаря предложению 7.2). Для каждого $k$ определим $m_{k} \in \mathbb{N}$ так, чтобы $e^{m_{k}-1} \leqslant t_{k} \leqslant e^{m_{k}}$. Тогда

$$
\begin{gathered}
\frac{1}{\log \left(1+t_{k}\right)} \int_{0}^{t_{k}} \mu_{s}(T) d s \leqslant \frac{1}{\log \left(1+e^{m_{k}-1}\right)} \int_{0}^{e^{m_{k}}} \mu_{s}(T) d s \\
=\frac{\log \left(1+e^{m_{k}}\right)}{\log \left(1+e^{m_{k}-1}\right)} \cdot \frac{1}{\log \left(1+e^{m_{k}}\right)} \int_{0}^{e^{m_{k}}} \mu_{s}(T) d s .
\end{gathered}
$$

Поскольку $\frac{\log \left(1+e^{m_{k}}\right)}{\log \left(1+e^{m_{k}-1}\right)} \rightarrow 1$, мы видим, что (12) следует из (13). Теорема 7.3 доказана.

Ответ на естественный вопрос, верно ли нормирующее свойство для класса следов Конна-Диксмье, приведен ниже. 
Tеорема 7.4 [60]. Пусть $T \in \mathscr{L}^{(1, \infty)}(\mathscr{N}, \tau)$. Расстояние от $T$ до подпространства $\mathscr{L}_{0}^{(1, \infty)}(\mathscr{N}, \tau)$ в норме $\|\cdot\|_{(1, \infty)}$ эквивалентно $\sup _{\omega} \tau_{\omega}(T)$, где супремум берется по всем сингулярным состояниям $\omega=\gamma \circ M$, где $\gamma$ - сингулярное состояние на $C_{b}[0, \infty)$.

Как и в предыдущем разделе, результаты, приведенные в теоремах 7.3 и 7.4, допускают обобщение на пространства Марцинкевича $M(\psi)(\mathscr{N}, \tau)$ с $\psi \in \Omega$, удовлетворяющим условию (4).

Мы закончим этот раздел замечанием о том, что еще не ясно, означает ли различие в результатах теорем 7.3 и 7.4, что множество всех следов Диксмье отлично от множества всех следов Конна-Диксмье.

\section{8. Фредгольмовы модули и спектральные тройки}

8.1. Обозначения и определения. Пусть $\mathscr{N}$ - полуконечная алгебра фон Неймана на сепарабельном гильбертовом пространстве $\mathscr{H}$ и $L_{p}(\mathscr{N}, \tau)-$ некоммутативное пространство $L_{p}$, ассоциированное с $(\mathscr{N}, \tau)$, где $\tau$ - точный, нормальный, полуконечный след на $\mathscr{N}$. Пусть $\mathscr{A}$ - унитальная банахова $*-$ алгебра, представленная в $\mathscr{N}$ при помощи непрерывного $*$-гомоморфизма $\pi$, который без потери общности можно предполагать точным. Если не возникает путаницы, мы будем опускать $\pi$ в обозначениях. Фундаментальные объекты нашего анализа объясняются в следующем определении.

Пусть $\mathscr{K}_{\mathscr{N}}-\tau$-компактные операторы в $\mathscr{N}$ (т.е. замкнутый по норме идеал, порожденный проекторами $E \in \mathscr{N}$ такими, что $\tau(E)<\infty)$.

ОПредЕЛЕНиЕ 8.1. (i) Полуконечная нечетная спектральная тройка ( $\mathscr{A}$, $\mathscr{H}, \mathscr{D})$ задается гильбертовым пространством $\mathscr{H}, *$-алгеброй $\mathscr{A} \subset \mathscr{N}$, где $\mathscr{N}$ - полуконечная алгебра фон Неймана, действующая в $\mathscr{H}$, и плотно определенным неограниченным самосопряженным оператором $\mathscr{D}$, присоединенным к $\mathscr{N}$, такими, что

1) $[\mathscr{D}, a]$ плотно определен и продолжается до ограниченного оператора для любого $a \in \mathscr{A}$;

2) $(\lambda-\mathscr{D})^{-1} \in \mathscr{K}_{\mathscr{N}}$ для любого $\lambda \notin \mathbb{R}$.

(ii) Скажем, что $(\mathscr{A}, \mathscr{H}, \mathscr{D})$ четна, если, дополнительно, существует такая $\mathbb{Z}_{2}$-градуировка, что $\mathscr{A}$ четна и $\mathscr{D}$ нечетен, т.е. существует такой оператор $\Gamma$, что $\Gamma=\Gamma^{*}, \Gamma^{2}=1, \Gamma a=a \Gamma$ для любого $a \in \mathscr{A}$ и $\mathscr{D} \Gamma+\Gamma \mathscr{D}=0$.

(iii) Если $\mathscr{I}$ - симметрично-нормированный идеал в $\mathscr{K}_{\mathscr{N}}$, то мы скажем, что спектральная тройка $(\mathscr{A}, \mathscr{N}, \mathscr{D}) \quad \mathscr{I}$-суммируема, если $\left(1+\mathscr{D}^{2}\right)^{-1 / 2} \in \mathscr{I}$.

ЗАмЕчАниЕ 8.2. В работе [22] используется другой термин для понятия, которое мы только что ввели, - спектральная тройка фон Неймана ("von Neumann spectral triple"). Имеются два наиболее важных специальных случая этого определения:

a) $\mathscr{I}$ - идеал $L^{p}$; в этом случае мы скажем, что $(\mathscr{A}, \mathscr{N}, \mathscr{D})$-суммируема,

b) случай, в котором впервые появился след Диксмье, а именно, $\mathscr{I}=\mathscr{L}^{p, \infty}$; в этом случае мы говорим, что $(\mathscr{A}, \mathscr{N}, \mathscr{D})$ является $(p, \infty)$-суммируемой. Третий случай базируется на понятии $\theta$-суммируемости (см., например, [65], [66], [7]) и не имеет отношения к нашему нынешнему обсуждению. 
ЗАМЕЧАНИЕ. В этой работе, для простоты изложения, мы будем иметь дело только с унитальными алгебрами $\mathscr{A} \subset \mathscr{N}$, причем единица в $\mathscr{A}$ совпадает с единицей в $\mathscr{N}$. Мы приняли следующее соглашение по поводу обозначений, в соответствии с контекстом, в котором мы работаем. Каллиграфическая буква $\mathscr{D}$ будет всегда обозначать неограниченный самосопряженный оператор, образующий часть полуконечной спектральной тройки $(\mathscr{A}, \mathscr{H}, \mathscr{D})$. Латинская буква $D$ будет обозначать самосопряженный оператор в гильбертовом пространстве, обычно с некоторыми дополнительными условиями.

В исходной работе по некоммутативной геометрии А. Конна [67] понятие спектральной тройки было введено как "неограниченный фредгольмов модуль" (см. также [7]). Изучение полуконечных спектральных троек было начато в [23] в контексте спектрального потока [68], [69] и проводилось далее в [22], концентрируясь на приложения к слоениям. В работе [67], основываясь на понятии модулей Каспарова, было введено понятие фредгольмова модуля. Это определение можно следующим образом обобщить на полуконечный случай.

ОПРЕДЕЛЕНИЕ 8.3 [23], [39]. Ограниченный $p$-суммируемый предмодуль Брейера-Фредгольма для $\mathscr{A}$ - это пара $\left(\mathscr{N}, F_{0}\right)$, где $F_{0}$ - ограниченный самосопряженный оператор в $\mathscr{N}$, удовлетворяющий условиям:

(1) $\left|\mathbf{1}-F_{0}^{2}\right|^{1 / 2}$ принадлежит $L_{p}(\mathscr{N}, \tau)$; и

(2) $\mathscr{A}_{p}:=\left\{a \in \mathscr{A}:\left[F_{0}, a\right] \in L_{p}(\mathscr{N}, \tau)\right\}$ - плотная *-подалгебра в $\mathscr{A}$.

Если $F_{0}^{2}=\mathbf{1}$, мы опустим приставку "пред-".

В частном случае, когда $\mathscr{N}=\mathscr{L}(\mathscr{H})$ и $\tau$ - стандартный след $\mathrm{Tr}$, мы опустим в определении слово "Брейер". В этом случае некоммутативное пространство $L_{p}$ совпадает с идеалом Шаттена-фон Неймана $\mathscr{C}_{p}$ и определение совпадает с определением 3 из [7] (с. 290).

\section{2. Сопоставление ограниченных и неограниченных модулей.}

Взаимосвязь между ограниченной и неограниченной картинами заслуживает некоторых комментариев. Имеется два подхода к этому вопросу - подход работы [70] (более подробно объясненный в [8]) и более общий подход, использующий теорию возмущений, предложенный в работе [28]. В случае четных спектральных троек этот вопрос был разрешен Конном (см. [67; I.6]). Пусть $D$ и $D_{0}-$ неограниченные самосопряженные операторы в $\mathscr{H}$, отличающиеся на ограниченный оператор из $\mathscr{N}$. Мы непосредственно исследуем отображение $\varphi$, определяемое формулой $\varphi(D)=D\left(1+D^{2}\right)^{-1 / 2}$, и разность $\varphi(D)-\varphi\left(D_{0}\right)$. В работе [28] было установлено несколько результатов, из которых наиболее близким к этому обзору является следующая теорема.

Теорема 8.4. При сделанных выше предположения на $D$ u $D_{0}$ u при $D-D_{0} \in \mathscr{N}$ u $1<p<\infty$ имеем

$\left\|\varphi(D)-\varphi\left(D_{0}\right)\right\|_{L_{p}(\mathscr{N}, \tau)} \leqslant Z_{p} \max \left\{\left\|D-D_{0}\right\|^{1 / 2},\left\|D-D_{0}\right\|\right\} \cdot\left\|\left(1+D^{2}\right)^{-1 / 2}\right\|_{L_{p}(\mathscr{N}, \tau)}$

при некоторой положительной постоянной $Z_{p}$, зависящая только от $p$.

Из этой теоремы (см. [70]) получается следующее утверждение.

СлеДСтвиЕ 8.5. Пусть $1<p<\infty u\left(\mathscr{A}, \mathscr{N}, \mathscr{D}_{0}\right)$ - нечетная полуконечная р-суммируемая спектральная тройка для банаховой *-алгебры. Тогда 
$\left(\mathscr{N}, \operatorname{sign}\left(\mathscr{D}_{0}\right)\right)$ - нечетный ограниченный $p$-суммируемый модуль БрейераФредголвма для $\mathscr{A}$.

\section{3. Дополнительная информация о полуконечных спектральных тройках.}

ОПрЕДЕЛЕНИЕ 8.6. Полуконечная спектральная тройка $(\mathscr{A}, \mathscr{H}, \mathscr{D})$ принадлежит классу $Q C^{k}$ при $k \geqslant 1$ (буква $Q$ заменяет слово “quantum"), если любой оператор $a \in \mathscr{A}$ вместе с оператором $[\mathscr{D}, a]$ принадлежат области определения $\delta^{k}$, где $\delta(T)=[|\mathscr{D}|, T]$ - частное дифференцирование на $\mathscr{N}$, определяемое $|\mathscr{D}|$.

ЗАмечАниЕ. (i) Здесь подразумевается, что наше обозначение аналогично классическому обозначению, но мы ввели букву $Q$ для того, чтобы не было путаницы между квантовой дифференцируемостью элемента $a \in \mathscr{A}$ и классической дифференцируемостью функций.

(ii) Под словами "частное дифференцирование" мы понимаем то, что $\delta$ определена на некоторой подалгебре в $\mathscr{N}$, которая не обязательно (слабо) плотна в $\mathscr{N}$. Точнее, $\operatorname{dom} \delta=\{T \in \mathscr{N}: \delta(T)$ ограничен $\}$.

НАБЛЮДЕНИЕ. Если $T \in \mathscr{N}$, можно показать, что $[|\mathscr{D}|, T]$ ограничен тогда и только тогда, когда $\left[\left(1+\mathscr{D}^{2}\right)^{1 / 2}, T\right]$ ограничен, показав, используя функицональное исчисление, что $|\mathscr{D}|-\left(1+\mathscr{D}^{2}\right)^{1 / 2}$ продолжается до ограниченного оператора в $\mathscr{N}$. Фактически, записывая $|\mathscr{D}|_{1}=\left(1+\mathscr{D}^{2}\right)^{1 / 2}$ и $\delta_{1}(T)=\left[|\mathscr{D}|_{1}, T\right]$, мы имеем $\operatorname{dom} \delta^{n}=\operatorname{dom} \delta_{1}^{n}$ для всех $n$.

ДокАЗАтельство. Пусть $f(\mathscr{D})=\left(1+\mathscr{D}^{2}\right)^{1 / 2}-|\mathscr{D}|$, тем самым, как было отмечено выше, оператор $f(\mathscr{D})$ продолжается до ограниченного оператора в $\mathscr{N}$. Поскольку оператор

$$
\delta_{1}(T)-\delta(T)=[f(\mathscr{D}), T]
$$

всегда ограничен, $\operatorname{dom} \delta=\operatorname{dom} \delta_{1}$. Теперь $\delta \delta_{1}=\delta_{1} \delta$, поэтому

$$
\begin{aligned}
\delta_{1}^{2}(T)-\delta^{2}(T) & =\delta_{1}\left(\delta_{1}(T)\right)-\delta_{1}(\delta(T))+\delta_{1}(\delta(T))-\delta(\delta(T)) \\
& =\left[f(\mathscr{D}), \delta_{1}(T)\right]+[f(\mathscr{D}), \delta(T)] .
\end{aligned}
$$

Оба слагаемых в правой части ограничены, поэтому $\operatorname{dom} \delta^{2}=\operatorname{dom} \delta_{1}^{2}$. Доказательство продолжается индукцией.

Таким образом, условие, определяющее класс $Q C^{k}$, можно заменить условием

$$
a,[\mathscr{D}, a] \in \bigcap_{0 \leqslant k \leqslant n} \operatorname{dom} \delta_{1}^{n} \quad \forall a \in \mathscr{A} .
$$

Это важно, так как мы нигде не предполагаем, ито $|\mathscr{D}|$ обратим.

Если $(\mathscr{A}, \mathscr{H}, \mathscr{D})$ - спектральная тройка класса $Q C^{k}$, то алгебру $\mathscr{A}$ можно наделить топологией, определяемой полунормами

$$
a \rightarrow\left\|\delta^{n}(a)\right\|+\left\|\delta^{n}([\mathscr{D}, a])\right\|, \quad n=0,1, \ldots, k .
$$

Согласно лемме 16 из [71], без потери общности можно предполагать, что $\mathscr{A}$ полна в получающейся топологии, переходя к пополнению, если это необходимо. Это пополнение инвариантно при голоморфном функциональном исчислении, тем самым, мы имеем осмысленную спектральную теорию и вложение 
$K_{*}(\mathscr{A}) \cong K_{*}(\overline{\mathscr{A}})$ при помощи включения, где $\overline{\mathscr{A}}-C^{*}$-пополнение алгебры $\mathscr{A}$. Спектральная тройка класса $Q C^{\infty}$ - это такая спектральная тройка, которая принадлежит $Q C^{k}$ при всех $k=1,2, \ldots$.

НАБЛЮДЕНИЕ. Если $T \in \mathscr{N} u[\mathscr{D}, T]$ ограничен,

ДокАЗАТЕЛЬство. Заметим, что оператор $\mathscr{D}$ присоединен к $\mathscr{N}$ и потому коммутирует со всеми проекторами, принадлежащими коммутанту алгебры $\mathscr{N}$, и коммутант алгебры $\mathscr{N}$ сохраняет область определения оператора $\mathscr{D}$. Таким образом, если $[\mathscr{D}, T]$ ограничен, он также коммутирует со всеми проекторами, принадлежащими коммутанту алгебры $\mathscr{N}$, и эти проекторы сохраняют область определения оператора $\mathscr{D}$ и поэтому $[\mathscr{D}, T] \in \mathscr{N}$.

Подобные замечания применимы к операторам $[|\mathscr{D}|, T],\left[\left(1+\mathscr{D}^{2}\right)^{1 / 2}, T\right]$ и таким комбинациям, как $\left[\mathscr{D}^{2}, T\right]\left(1+\mathscr{D}^{2}\right)^{-1 / 2}$. Чтобы упростить обозначения, мы будем часто писать просто $\mathscr{L}^{1}$ для обозначения идеала ядерных операторов и обозначать норму в $\mathscr{L}^{1}$ через $\|\cdot\|_{1}$. Заметим, что в случае, когда $\mathscr{N} \neq \mathscr{L}(\mathscr{H})$, идеал $\mathscr{L}^{1}$ не обязательно является полным в этой норме, но он является полным по отношению к норме $\|\cdot\|_{1}+\|\cdot\|_{\infty}$, где $\|\cdot\|_{\infty}$ обозначает равномерную норму.

8.4. Суммируемость и размерность. Условие конечной суммируемости спектральной тройки задает полуплоскость, в которой функция

$$
z \mapsto \tau\left(\left(1+\mathscr{D}^{2}\right)^{-z}\right)
$$

корректно определена и голоморфна.

ОПРЕДЕЛЕНИЕ 8.7 . Если $(\mathscr{A}, \mathscr{H}, \mathscr{D})$ - спектральная тройка класса $Q C^{\infty}$, мы назовем число

$$
p=\inf \left\{q \in \mathbb{R}: \tau\left(\left(1+\mathscr{D}^{2}\right)^{-q / 2}\right)<\infty\right\}
$$

спектралъной размерностъю тройки $(\mathscr{A}, \mathscr{H}, \mathscr{D})$.

\section{9. Спектральный поток}

Одной из основных мотивировок для распространения исследований спектральных троек в ситуацию полуконечных алгебр фон Неймана является изучение спектрального потока типа $I I$ (это понятие принадлежит Дж. Филлипсу [72], [73]). Пусть $\pi: \mathscr{N} \rightarrow \mathscr{N} / \mathscr{K}_{\mathscr{N}}-$ каноническое отображение. Оператор Брейера-Фредгольма - это такой оператор, который переходит в обратимый оператор под действием отображения $\pi$. Полное обсуждение теории БрейераФредгольма в полуконечной алгебре фон Неймана содержится в работе [26], что расширяет обсуждение, приведенное в приложении к работе [74] и в [75], [76]. Как обычно, $D$ - неограниченный плотно определенный самосопряженный оператор Брейера-Фредгольма в $\mathscr{H}$ (в том смысле, что $D\left(1+D^{2}\right)^{-1 / 2}$ ограничен и является оператором Брейера-Фредгольма в $\mathscr{N})$ такой, что $\left(1+D^{2}\right)^{-1 / 2} \in \mathscr{K}_{\mathscr{N}}$. Для унитарного оператора $u \in \mathscr{N}$ такого, что $[D, u]$ - ограниченный оператор, путь

$$
D_{t}^{u}:=(1-t) D+t u D u^{*}
$$


состоящий из неограниченных самосопряженных операторов Брейера-Фредгольма, непрерывен в том смысле, что

$$
F_{t}^{u}:=D_{t}^{u}\left(1+\left(D_{t}^{u}\right)^{2}\right)^{-\frac{1}{2}}
$$

есть непрерывный по норме путь самосопряженных операторов Брейера-Фредгольма в $\mathscr{N}$ [23]. Напомним, что индекс Брейера-Фредгольма оператора Брейера-Фредгольма $F$ определяется формулой

$$
\operatorname{ind}(F)=\tau\left(Q_{\text {ker } F}\right)-\tau\left(Q_{\text {coker } F}\right),
$$

где $Q_{\text {ker } F}$ и $Q_{\text {coker } F}$ - проекторы на ядро и коядро оператора $F$.

ОПРЕДЕЛЕНИЕ 9.1. Если $\left\{F_{t}\right\}$ - непрерывный путь, состоящий из самосопряженных операторов Брейера-Фредгольма в $\mathscr{N}$, то определение спектрального потока этого пути, $\operatorname{sf}\left(\left\{F_{t}\right\}\right)$, основано на следующей последовательности наблюдений, сделанных в [77], [78] и [73] (см. также [79], [80]).

1. Несмотря на то, что функция $t \mapsto \operatorname{sign}\left(F_{t}\right)$ типично разрывна, как и проекторнозначное отображение $t \mapsto P_{t}=\frac{1}{2}\left(\operatorname{sign}\left(F_{t}\right)+1\right)$, где $F_{t}=2 P_{t}-1$ и $P_{t}$ - неотрицательный спектральный проектор, функция $t \mapsto \pi\left(P_{t}\right)$ непрерывна.

2. Если $P$ и $Q$ - проекторы в $\mathscr{N}$, то $P Q: Q \mathscr{H} \rightarrow P \mathscr{H}$ является оператором Брейера-Фредгольма тогда и только тогда, когда $\|\pi(P)-\pi(Q)\|<1$, причем в этом случае корректно определен ind $(P Q) \in \mathbb{R}$.

3. Если мы разобьем интервал изменения параметра семейства $\left\{F_{t}\right\}$ таким образом, что $\pi\left(P_{t}\right)$ не сильно изменяется по норме на каждом подынтервале разбиения, то

$$
\operatorname{sf}\left(\left\{F_{t}\right\}\right):=\sum_{i=1}^{n} \operatorname{ind}\left(P_{t_{i-1}} P_{t_{i}}\right)
$$

является корректно определенным и инвариантным при гомотопии пути числом, которое согласуется с обычным понятием спектрального потока в случае типа $I_{\infty}$.

4. Для $D$ и $u$ как выше мы определим спектралъный поток пути $D_{t}^{u}:=$ $(1-t) D+t u D u^{*}$ как спектральный поток пути $F_{t}$, где $F_{t}=D_{t}^{u}\left(1+\left(D_{t}^{u}\right)^{2}\right)^{-\frac{1}{2}}$. Мы обозначим это число через

$$
\operatorname{sf}\left(D, u D u^{*}\right)=\operatorname{sf}\left(\left\{F_{t}\right\}\right)
$$

и заметим, что оно является целым числом в случае $\mathscr{N}=\mathscr{L}(\mathscr{H})$ и вещественным числом в общем полуконечном случае.

Частные случаи спектрального потока в полуконечной алгебре фон Неймана обсуждались в работах [81], [77], [78], [82], [72], [73].

Пусть $P$ обозначает проектор на неотрицательное спектральное подпространство оператора $D$. Спектральный поток вдоль $\left\{D_{t}^{u}\right\}$ равен $\operatorname{sf}\left(\left\{F_{t}\right\}\right)$ и, согласно [23], это число совпадает с индексом Брейера-Фредгольма оператора $P u P u^{*}$. (Заметим, что $\operatorname{sign} F_{1}^{u}=2 u P u^{*}-1$ и что для этого специального пути мы имеем, что $P-u P u^{*}$ компактен, поэтому $P u P u^{*}$, конечно, является оператором Брейера-Фредгольма как оператор из $u P u^{*} \mathscr{H}$ в $P \mathscr{H}$.) Теперь [74; приложение В] мы имеем ind $\left(P u P u^{*}\right)=\operatorname{ind}(P u P)$. 
Оператор $P$ Р известен как обобщенный тёплицев оператор. Формулы для его индекса в терминах следа Диксмье обсуждаются в разделах 13 и 16. Эти формулы в терминах следа Диксмье следуют из аналитических формул для спектрального потока (открытых в работах [83], [23], [24]), которые мы сейчас объясним.

9.1. Формулы для спектрального потока. Теперь мы введем формулу для спектрального потока, полученную А. Кери и Дж. Филлипсом [23], [24]. Эта формула, отправляясь от полуконечной спектральной тройки $(\mathscr{A}, \mathscr{H}, \mathscr{D})$, вычисляет спектральный поток от $\mathscr{D}$ до $u \mathscr{D} u^{*}$ (где $u \in \mathscr{A}$ - такой унитарный элемент, что $[\mathscr{D}, u]$ ограничен) в случае, когда $(\mathscr{A}, \mathscr{H}, \mathscr{D})$ имеет размерность $p \geqslant 1$. Таким образом, для любого $n>p$, согласно обобщению теоремы 9.3 из [24] на случай общих полуконечных алгебр фон Неймана (см. [27]), имеем:

$$
\operatorname{sf}\left(\mathscr{D}, u \mathscr{D} u^{*}\right)=\frac{1}{C_{n / 2}} \int_{0}^{1} \tau\left(u\left[\mathscr{D}, u^{*}\right]\left(1+\left(\mathscr{D}+t u\left[\mathscr{D}, u^{*}\right]\right)^{2}\right)^{-n / 2}\right) d t
$$

где

$$
C_{n / 2}=\int_{-\infty}^{\infty}\left(1+x^{2}\right)^{-n / 2} d x .
$$

Это вещественное число $\operatorname{sf}\left(\mathscr{D}, u \mathscr{D} u^{*}\right)$ восстанавливает спаривание класса $K$ гомологий $[\mathscr{D}]$ алгебры $\mathscr{A}$ с классом $[u]$ в $K_{1}(\mathscr{A})$ (см. ниже). Имеется геометрическая точка зрения на эту формулу, принадлежащая Э. Гецлеру [83]. Как показано в [24], функционал $X \mapsto \tau\left(X\left(1+(\mathscr{D}+X)^{2}\right)^{-n / 2}\right)$ на $\mathscr{N}_{s a}=\{T \in \mathscr{N}$ : $\left.T=T^{*}\right\}$ определяет точную 1 -форму на аффинном пространстве $\mathscr{D}+\mathscr{N}_{s a}$. Таким образом, формула (15) представляет интеграл этой 1-формы вдоль пути $\left\{\mathscr{D}_{t}=(1-t) \mathscr{D}+t u \mathscr{D} u^{*}\right\}$ при условии, что $\dot{\mathscr{D}}_{t}=u\left[\mathscr{D}, u^{*}\right]$ понимается как вектор, касательный к этому пути. Более того, эта формула инвариантна при растяжениях. Под этим мы понимаем то, что если заменить $\mathscr{D}$ на $\varepsilon \mathscr{D}$ при $\varepsilon>0$ в правой части формулы (15), то левая часть не изменится, как это видно из определения спектрального потока. Это справедливо потому, что спектральный поток включает в себя только фазу оператора $\mathscr{D}$, которая совпадает с фазой оператора $\varepsilon \mathscr{D}$.

9.2. Связи с циклическими когомологиями. Чтобы поместить обсуждения в правильный контекст и приготовиться к дальнейшим приложениям, необходимо обсудить некоторые аспекты теории циклических когомологий. Мы будем использовать нормализованный $(b, B)$-бикомплекс (см. [7], [84]).

Введем следующие линейные пространства. Пусть $C_{m}=\mathscr{A} \otimes \overline{\mathscr{A}}^{\otimes m}$, где $\overline{\mathscr{A}}$ - факторпространство $\mathscr{A} / \mathbb{C} I(I-$ единичный элемент алгебры $\mathscr{A})$ и (предполагая без потери общности, что $\mathscr{A}$ полна в $\delta$-топологии) мы используем проективное тензорное произведение. Пусть $C^{m}=\operatorname{Hom}\left(C_{m}, \mathbb{C}\right)$ - линейное пространство непрерывных полилинейных функционалов на $C_{m}$. Можно определить $(b, B)$-бикомплекс, используя эти пространства (в противоположность к $C_{m}=\mathscr{A}^{\otimes m+1}$ и т.д.), причем получающиеся таким образом когомологии будут теми же самыми. Это справедливо, поскольку бикомплекс, определяемый при помощи $\mathscr{A} \otimes \overline{\mathscr{A}}^{\otimes m}$, квазиизоморфен бикомплексу, определяемому при помощи $\mathscr{A} \otimes \mathscr{A}^{\otimes m}$. 
Нормализованная $(b, B)$-коцепь $\varphi$ есть конечная совокупность непрерывных полилинейных функционалов на $\mathscr{A}$,

$$
\varphi=\left\{\varphi_{m}\right\}_{m=1, \ldots, M}, \quad \text { где } \quad \varphi_{m} \in C^{m} .
$$

Она является (нормализованным) $(b, B)$-коциклом, если для любого $m b \varphi_{m}+$ $B \varphi_{m+2}=0$, где $b: C^{m} \rightarrow C^{m+1}, B: C^{m} \rightarrow C^{m-1}$ - кограничные операторы, задаваемые формулами

$$
\begin{gathered}
\left(B \varphi_{m}\right)\left(a_{0}, a_{1}, \ldots, a_{m-1}\right)= \\
\sum_{j=0}^{m-1}(-1)^{(m-1) j} \varphi_{m}\left(1, a_{j}, a_{j+1}, \ldots, a_{m-1}, a_{0}, \ldots, a_{j-1}\right) \\
\left(b \varphi_{m-2}\right)\left(a_{0}, a_{1}, \ldots, a_{m-1}\right)= \\
\sum_{j=0}^{m-2}(-1)^{j} \varphi_{m-2}\left(a_{0}, a_{1}, \ldots, a_{j} a_{j+1}, \ldots, a_{m-1}\right) \\
\quad+(-1)^{m-1} \varphi_{m-2}\left(a_{m-1} a_{0}, a_{1}, \ldots, a_{m-2}\right) .
\end{gathered}
$$

Для краткости мы далее будем писать $(b+B) \varphi=0$. Нормализованный коцикл, рассматриваемый как функционал на $\mathscr{A}^{\otimes m+1}$, будет удовлетворять условию $\varphi\left(a_{0}, a_{1}, \ldots, a_{n}\right)=0$, если хотя бы одно $a_{j}=1$ при $j \geqslant 1$. Коцепь называется нечетной (четной), если $\left\{\varphi_{m}\right\}=0$ для четных (нечетных) $m$.

Аналогично, $\left(b^{T}, B^{T}\right)$-цепь, $c$ есть (возможно, бесконечная) совокупность $c=$ $\left\{c_{m}\right\}_{m=1,2, \ldots}$, где $c_{m} \in C_{m} . \quad(b, B)$-цепь $\left\{c_{m}\right\}$ является $\left(b^{T}, B^{T}\right)$-циклом, если $b^{T} c_{m+2}+B^{T} c_{m}=0$ для любого $m$. Более кратко будем писать $\left(b^{T}+B^{T}\right) c=0$. Здесь $b^{T}, B^{T}$ - граничные операторы в циклических гомологиях, являющиеся транспонированными к кограничным операторам $b, B$ в следующем смысле.

Спаривание между $(b, B)$-коцепью $\varphi=\left\{\varphi_{m}\right\}_{m=1}^{M}$ и $\left(b^{T}, B^{T}\right)$-цепью $c=\left\{c_{m}\right\}$ задается формулой

$$
\langle\varphi, c\rangle=\sum_{m=1}^{M} \varphi_{m}\left(c_{m}\right) .
$$

Это спаривание удовлетворяет условию

$$
\langle(b+B) \varphi, c\rangle=\left\langle\varphi,\left(b^{T}+B^{T}\right) c\right\rangle .
$$

Имеются соотношения

$$
b^{2}=B^{2}=0=b B+B b=(b+B)^{2},
$$

таким образом, можно определить циклические когомологии алгебры $\mathscr{A}$ как когомологии тотального $(b, B)$-комплекса.

В этом обзоре нашим основным приложением следа Диксмье к циклическим когомологиям будет обсуждение в разделе 17 формулы А. Конна для класса Хохшильда характера Черна. Здесь мы используем ненормализованный комплекс $\widetilde{C}^{m}=\mathscr{A}^{\otimes m+1}$. Кограница Хохшильда $b$ на $\widetilde{C}^{m}$ определяется по той же формуле (16). В таком случае когомологии Хохшильда, обозначаемые через $H H^{*}\left(\mathscr{A}, \mathscr{A}^{*}\right)$, суть когомологии комплекса $\left(\widetilde{C}^{*}(\mathscr{A}), b\right)$.

Граница Хохшильда на комплексе $\widetilde{C}_{m}=\mathscr{A}^{\otimes m+1}$ есть оператор $b^{T}$. Мы скажем, что $c$ есть цикл Хохшильда, если $b^{T} c=0$. Когда гомологии Хохшильда корректно определены, мы обозначим их через $H H_{*}(\mathscr{A})$. 
Спектральный поток (в случае типа $I$ ) можно интерпретировать как спаривание между нечетным классом в $K$-теории, представляемым унитарным элементом $u$, и нечетным классом в $K$-гомологиях, представляемым спектральной тройкой $(\mathscr{A}, \mathscr{H}, \mathscr{D})[7 ;$ гл. III, IV]. Такая точка зрения также имеет смысл в общей полуконечной постановке, хотя необходимо подходящим образом интерпретировать $K$-гомологии [25], [24]. Особая черта работы [7] состоит в переносе $K$-теоретического спаривания в циклическую теорию для того, чтобы получить теоремы об индексе. Подходящему представителю класса в $K$-теории (соответственно класса в $K$-гомологиях) ставится в соответствие класс в периодических циклических гомологиях (соответственно класс в периодических циклических когомологиях), называемый в обоих случаях классом Черна. (Мы не будем отвлекаться здесь на обсуждение периодической теории и оператора периодичности, ссылаясь вместо этого на работы [7] и [8].) Главный результат состоит тогда в том, что

$$
\operatorname{sf}\left(\mathscr{D}, u \mathscr{D} u^{*}\right)=\langle[u],[(\mathscr{A}, \mathscr{H}, \mathscr{D})]\rangle=-\frac{1}{\sqrt{2 \pi i}}\left\langle\left[\mathrm{Ch}_{*}(u)\right],\left[\mathrm{Ch}^{*}(\mathscr{A}, \mathscr{H}, \mathscr{D})\right]\right\rangle,
$$

где $[u] \in K_{1}(\mathscr{A})$ - класс в $K$-теории с представителем $u$ и $[(\mathscr{A}, \mathscr{H}, \mathscr{D})]-$ класс в $K$-гомологиях, задаваемый спектральной тройкой $(\mathscr{A}, \mathscr{H}, \mathscr{D})$. В правой части $\mathrm{Ch}_{*}(u)$ - характер Черна элемента $u$. Напомним, что характер Черна унитарного элемента $u$ есть (бесконечная) совокупность нечетных цепей $\mathrm{Ch}_{2 j+1}(u)$, удовлетворяющая условию $b \mathrm{Ch}_{2 j+3}(u)+B \mathrm{Ch}_{2 j+1}(u)=0$,

$$
\mathrm{Ch}_{2 j+1}(u)=(-1)^{j} j ! \underbrace{u^{*} \otimes u \otimes \cdots \otimes u^{*} \otimes u}_{2 j+2 \text { члена }} .
$$

Мы использовали обозначение $\left[\mathrm{Ch}_{*}(u)\right]$ для класса в периодических циклических гомологиях. Подобным образом, $\left[\mathrm{Ch}^{*}(\mathscr{A}, \mathscr{H}, \mathscr{D})\right]$ есть класс характера Черна тройки $(\mathscr{A}, \mathscr{H}, \mathscr{D})$ в периодических циклических когомологиях.

\section{0. След Диксмье и вычеты дзета-функции}

Многие приложения следа Диксмье основываются на том, что его можно вычислить, беря вычет ассоциированной дзета-функции. Главный результат в этом направлении в случае типа $I$ есть предложение IV 2.4 работы [7]. Недавние продвижения [29] распространили этот результат на общий случай полуконечной алгебры фон Неймана.

10.1. Предварительные сведения. Прежде всего, полезно иметь следующую оценку сингулярных чисел операторов из $\mathscr{L}^{(1, \infty)}$.

Лемма 10.1. Для положительного оператора $T \in \mathscr{L}^{(1, \infty)}$ существует такая постоянная $K>0$, что для каждого $p \geqslant 1$

$$
\int_{0}^{t} \mu_{s}(T)^{p} d s \leqslant K^{p} \int_{0}^{t} \frac{1}{(s+1)^{p}} d s .
$$

ДоказАтельство. Согласно [47; лемма 2.5 (iv)], для любого $0 \leqslant T \in \mathscr{N}$ и для всех непрерывных возрастающих функций $f$ на $[0, \infty)$ с $f(0) \geqslant 0$ имеем 
$\mu_{s}(f(T))=f\left(\mu_{s}(T)\right)$ для всех $s>0$. Комбинируя этот факт с хорошо известным результатом Харди-Литлвуда-Пойя (см., например, [48; лемма 4.1]), мы видим, что если $T_{1} \prec \prec T_{2}, 0 \leqslant T_{1}, T_{2} \in \mathscr{N}$, то $T_{1}^{p} \prec \prec T_{2}^{p}$ для всех $p \in(1, \infty)$. Теперь по определению пространства $\mathscr{L}^{(1, \infty)}$ сингулярные числа оператора $T$ удовлетворяют условию $\int_{0}^{t} \mu_{s}(T) d s=O(\log t)$, тем самым, при некотором $K>0$

$$
\int_{0}^{t} \mu_{s}(T) d s \leqslant K \int_{0}^{t} \frac{1}{(s+1)} d s \quad \forall t>0 .
$$

Другими словами, $\mu_{s}(T) \prec \prec K /(1+s)$ и утверждение леммы следует немедленно.

В следующей теореме мы используем состояния, инвариантные при растяжениях, из теоремы 4.7.

Теорема $10.2\left({ }^{*}\right.$-слабая теорема Караматы). Пусть $\widetilde{\omega} \in L_{\infty}(\mathbb{R})^{*}-$ cocmoяние, инвариантное при растяжениях, и $\beta$ - вещественнозначная возрастающая непрерывная справа функиия на $\mathbb{R}_{+}$, равная нулю в нуле и такая, что интеграл

$$
h(r)=\int_{0}^{\infty} e^{-\frac{t}{r}} d \beta(t)
$$

сходится для всех $r>0$ и существует предел $C={\widetilde{\omega-\lim _{r \rightarrow \infty}}}_{r} \frac{1}{r} h(r)$. Тогда

$$
\overbrace{r \rightarrow \infty}^{\omega-\lim } \frac{1}{r} h(r)=\overbrace{t \rightarrow \infty}^{\omega-\lim } \frac{\beta(t)}{t} .
$$

ЗАмечАниЕ 10.3. Классическая теорема Караматы утверждает, в обозначениях данной теоремы, что если обычный предел $\lim _{r \rightarrow \infty} \frac{1}{r} h(r)=C$ существует, то $C=\lim _{t \rightarrow \infty} \frac{\beta(t)}{t}$. Доказательство этого классического результата получается заменой повсюду в доказательстве теоремы $10.2 \widetilde{\omega-\lim }$ обычным пределом.

ДокАЗАТЕЛьСтво. Пусть

$$
g(x)= \begin{cases}x^{-1} & \text { при } e^{-1} \leqslant x \leqslant 1, \\ 0 & \text { при } 0 \leqslant x<e^{-1},\end{cases}
$$

тем самым, функция $g$ непрерывна справа в $e^{-1}$. Тогда при $r>0$ функция $t \rightarrow e^{-t / r} g\left(e^{-t / r}\right)$ непрерывна слева при $t=r$. Таким образом, интеграл Римана-Стилтьеса

$$
\int_{0}^{\infty} e^{-t / r} g\left(e^{-t / r}\right) d \beta(t)
$$

существует для любого $r>0$. Мы утверждаем, что для любого многочлена $p$

$$
\widetilde{\omega-\lim _{r \rightarrow \infty}} \frac{1}{r} \int_{0}^{\infty} e^{-t / r} p\left(e^{-t / r}\right) d \beta(t)=C \int_{0}^{\infty} e^{-t} p\left(e^{-t}\right) d t .
$$


Чтобы доказать это равенство, сначала установим его для $p(x)=x^{n}$. Имеем

$$
\frac{1}{r} \int_{0}^{\infty} e^{-t / r} e^{-n t / r} d \beta(t)=\frac{1}{r} \int_{0}^{\infty} e^{-(n+1) t / r} d \beta(t) .
$$

Поэтому

$$
\frac{1}{n+1} \overbrace{r \rightarrow \infty}^{\omega-\lim _{r}} \frac{1}{r /(n+1)} \int_{0}^{\infty} e^{-(n+1) t / r} d \beta(t)=\frac{C}{n+1}
$$

в силу инвариантности $\widetilde{\omega}$ при растяжениях. Таким образом,

$$
\varlimsup_{r \rightarrow \infty}^{\omega-\lim } \frac{1}{r} \int_{0}^{\infty} e^{-t / r} e^{-n t / r} d \beta(t)=C \int_{0}^{\infty} e^{-t}\left(e^{-t}\right)^{n} d t .
$$

Поскольку $\widetilde{\omega}$ линейно, отсюда следует справедливость утверждения для всех $p$.

Выберем такие последовательности многочленов $\left\{p_{n}\right\},\left\{P_{n}\right\}$, что для всех $x \in[0,1]$

$$
-1 \leqslant p_{n}(x) \leqslant g(x) \leqslant P_{n}(x) \leqslant 3
$$

и $p_{n}$ и $P_{n}$ сходятся почти всюду к $g(x)$. Тогда, поскольку $\widetilde{\omega}$ положительно, оно сохраняет порядок:

$$
\begin{aligned}
C \int_{0}^{\infty} & e^{-t} p_{n}\left(e^{-t}\right) d t=\widetilde{\omega-\lim } \frac{1}{r} \int_{0}^{\infty} e^{-t / r} p_{n}\left(e^{-t / r}\right) d \beta(t) \\
& \approx \widetilde{\omega-\lim } \frac{1}{r} \int_{0}^{\infty} e^{-t / r} g\left(e^{-t / r}\right) d \beta(t) \leqslant \cdots \leqslant C \int_{0}^{\infty} e^{-t} P_{n}\left(e^{-t}\right) d t .
\end{aligned}
$$

Согласно теореме Лебега о мажорируемой сходимости, как $\int_{0}^{\infty} e^{-t} p_{n}\left(e^{-t}\right) d t$, так и $\int_{0}^{\infty} e^{-t} P_{n}\left(e^{-t}\right) d t$ сходятся к $\int_{0}^{\infty} e^{-t} g\left(e^{-t}\right) d t$ при $n \rightarrow \infty$. Но прямое вычисление дает, что $\int_{0}^{\infty} e^{-t} g\left(e^{-t}\right) d t=1$ и

$$
\int_{0}^{\infty} e^{-t / r} g\left(e^{-t / r}\right) d \beta(t)=\beta(r) .
$$

Следовательно,

$$
C=\widetilde{\omega-\lim _{r \rightarrow \infty}} \frac{1}{r} \int_{0}^{\infty} e^{-t / r} g\left(e^{-t / r}\right) d \beta(t)=\widetilde{\omega-\lim } \frac{\beta(r)}{r} .
$$

Теорема 10.2 доказана.

Напомним, что для любого $\tau$-измеримого оператора $T$ его функция распределения определяется формулой

$$
\lambda_{t}(T):=\tau\left(\chi_{(t, \infty)}(|T|)\right), \quad t>0,
$$

где $\chi_{(t, \infty)}(|T|)$ - спектральный проектор оператора $|T|$, соответствующий интервалу $(t, \infty)$ (см. [47]). Согласно предложению 2.2 в [47],

$$
\mu_{s}(T)=\inf \left\{t \geqslant 0: \lambda_{t}(T) \leqslant s\right\},
$$


откуда мы заключаем, что для любого $\tau$-измеримого оператора $T$ функция распределения $\lambda_{(\cdot)}(T)$ совпадает с (классической) функцией распределения функции $\mu_{(\cdot)}(T)$. Из этой формулы и того факта, что $\lambda$ непрерывна справа, можно легко увидеть, что для $t>0, s>0$

$$
s \geqslant \lambda_{t} \Longleftrightarrow \mu_{s} \leqslant t
$$

или, эквивалентно,

$$
s<\lambda_{t} \Longleftrightarrow \mu_{s}>t
$$

При помощи замечания 3.3 из [47] отсюда следует, что

$$
\int_{0}^{\lambda_{t}} \mu_{s}(T) d s=\int_{\left[0, \lambda_{t}\right)} \mu_{s}(T) d s=\tau\left(|T| \chi_{(t, \infty)}(|T|)\right), \quad t>0 .
$$

Лемма 10.4. Для $T \in \mathscr{L}^{(1, \infty)}$ u $C>\|T\|_{(1, \infty)}$ мы имеем при больиих $t$

$$
\lambda_{\frac{1}{t}}(T) \leqslant C t \log t
$$

ДокАЗАТЕЛЬСтво. Предположим, что это не так и существуют такие $t_{n} \uparrow \infty$, что $\lambda_{\frac{1}{t_{n}}}(T)>C t_{n} \log t_{n}$ и, тем самым, для $s \leqslant C t_{n} \log t_{n}$ мы имеем $\mu_{s}(T) \geqslant \mu_{C t_{n}} \log t_{n}(T)>\frac{1}{t_{n}}$. Тогда для достаточно больших $n$

$$
\int_{0}^{C t_{n} \log t_{n}} \mu_{s}(T) d s>\frac{1}{t_{n}} \cdot C t_{n} \log t_{n}=C \log t_{n} .
$$

Выберем $\delta>0$ так, что $C-\delta>\|T\|_{(1, \infty)}$. Тогда для достаточно больших $n$

$$
\begin{aligned}
C \log t_{n} & =(C-\delta) \log t_{n}+\delta \log t_{n}>\|T\|_{(1, \infty)} \log \left(C t_{n}\right)+\|T\|_{(1, \infty)} \log \log \left(t_{n}+1\right) \\
& =\|T\|_{(1, \infty)} \log \left(C t_{n} \log \left(t_{n}+1\right)\right) .
\end{aligned}
$$

Это противоречит неравенству $\int_{0}^{t} \mu_{s}(T) d s \leqslant\|T\|_{(1, \infty)} \log (t+1)$, которое справедливо для любого $t>0$ в силу определения нормы в $\mathscr{L}^{(1, \infty)}$. Лемма 10.4 доказана.

Утверждение, в чем-то похожее на приведенное ниже предложение 10.5, сформулировано в [36] и снабжено некорректным доказательством. Мы используем другой подход.

ПрЕДЛОЖЕНИЕ 10.5. Пусть $0 \leqslant T \in \mathscr{L}^{(1, \infty)}$, a $\omega$ - состояние на $L_{\infty}\left(\mathbb{R}_{+}^{*}\right)$, удовлетворяющее всем условиям следствия 4.8. Для любого $C>0$

$$
\begin{aligned}
\tau_{\omega}(T) & =\omega-\lim _{t \rightarrow \infty} \frac{1}{\log (1+t)} \int_{0}^{t} \mu_{s}(T) d s=\omega-\lim \frac{1}{\log (1+t)} \tau\left(T \chi_{\left(\frac{1}{t}, \infty\right)}(T)\right) \\
& =\omega-\lim \frac{1}{\log (1+t)} \int_{0}^{C t \log t} \mu_{s}(T) d s,
\end{aligned}
$$

и если один из $\omega$-пределов является настоящим пределом, то тогда это верно и для других пределов. 
ДокАзАтЕЛЬство. Прежде всего, заметим, что

$$
\int_{0}^{t} \mu_{s}(T) d s \leqslant \int_{0}^{\lambda_{\frac{1}{t}}(T)} \mu_{s}(T) d s+1, \quad t>0 .
$$

Действительно, приведенное выше неравенство тривиально имеет место, если $t \leqslant \lambda_{\frac{1}{t}}(T)$. Если $t>\lambda_{\frac{1}{t}}(T)$, то

$$
\int_{0}^{t} \mu_{s}(T) d s=\int_{0}^{\lambda_{\frac{1}{t}}(T)} \mu_{s}(T) d s+\int_{\lambda_{\frac{1}{t}}(T)}^{t} \mu_{s}(T) d s
$$

Из неравенства $s>\lambda_{\frac{1}{t}}(T)$ вытекает, что $\mu_{s}(T) \leqslant \frac{1}{t}$, поэтому мы имеем

$$
\int_{0}^{t} \mu_{s}(T) d s \leqslant \int_{0}^{\lambda_{\frac{1}{t}}(T)} \mu_{s}(T) d s+\frac{1}{t}\left(t-\lambda_{\frac{1}{t}}(T)\right) \leqslant \int_{0}^{\lambda_{\frac{1}{t}}(T)} \mu_{s}(T) d s+1 .
$$

Используя это наблюдение и приведенную выше лемму, мы видим, что для $C>\|T\|_{(1, \infty)}$ и для любого фиксированного $\alpha>1$ при больших $t$

$$
\int_{0}^{t} \mu_{s}(T) d s \leqslant \int_{0}^{\lambda_{\frac{1}{t}}(T)} \mu_{s}(T) d s+1 \leqslant \int_{0}^{C t \log t} \mu_{s}(T) d s+1 \leqslant \int_{0}^{t^{\alpha}} \mu_{s}(T) d s+1
$$

и, тем самым, при больших $t$

$$
\begin{aligned}
\frac{1}{\log (1+t)} \int_{0}^{t} \mu_{s}(T) d s & \leqslant \frac{1}{\log (1+t)}\left(\int_{0}^{\lambda_{\frac{1}{t}}(T)} \mu_{s}(T) d s+1\right) \\
& \leqslant \frac{1}{\log (1+t)}\left(\int_{0}^{C t \log t} \mu_{s}(T) d s+1\right) \\
& \leqslant \frac{\log \left(1+t^{\alpha}\right)}{\log (1+t) \log \left(1+t^{\alpha}\right)}\left(\int_{0}^{t^{\alpha}} \mu_{s}(T) d s+1\right)
\end{aligned}
$$

Взяв $\omega$-предел, мы получаем

$$
\begin{aligned}
\tau_{\omega}(T) & \leqslant \omega_{t \rightarrow \infty} \frac{1}{\log (1+t)} \int_{0}^{\lambda_{\frac{1}{t}}(T)} \mu_{s}(T) d s \\
& \leqslant \omega-\lim \frac{1}{\log (1+t)} \int_{0}^{C t \log t} \mu_{s}(T) d s \\
& \leqslant \omega-\lim \frac{\alpha}{\log \left(1+t^{\alpha}\right)} \int_{0}^{t^{\alpha}} \mu_{s}(T) d s=\alpha \tau_{\omega}(T),
\end{aligned}
$$

где последнее равенство использует следствие 4.8 (4). Так как это справедливо для всех $\alpha>1$, то, используя (18), мы получаем утверждение для $\omega$-пределов и при $C>\|T\|_{(1, \infty)}$. Для произвольного $0<C \leqslant\|T\|_{(1, \infty)}$ утверждение следует немедленно, если заметить, что для $C^{\prime}>\|T\|_{(1, \infty)}$ мы имеем при больших $t$

$$
\int_{0}^{t} \mu_{s}(T) d s \leqslant \int_{0}^{C t \log t} \mu_{s}(T) d s \leqslant \int_{0}^{C^{\prime} t \log t} \mu_{s}(T) d s .
$$


Чтобы доказать последнее утверждение предложения, предположим, что

$$
\lim _{t \rightarrow \infty} \frac{1}{\log (1+t)} \int_{0}^{t} \mu_{s}(T) d s=A .
$$

Тогда, используя приведенные выше рассуждения, мы имеем

$$
A \leqslant \liminf _{t \rightarrow \infty} \frac{1}{\log (1+t)} \tau\left(T \chi_{\left(\frac{1}{t}, \infty\right)}(T)\right) \leqslant \limsup _{t \rightarrow \infty} \frac{1}{\log (1+t)} \tau\left(T \chi_{\left(\frac{1}{t}, \infty\right)}(T)\right) \leqslant \alpha A
$$

для всех $\alpha>1$, и, следовательно,

$$
\lim _{t \rightarrow \infty} \frac{1}{\log (1+t)} \tau\left(T \chi_{\left(\frac{1}{t}, \infty\right)}(T)\right)=A
$$

С другой стороны, если предел

$$
\lim _{t \rightarrow \infty} \frac{1}{\log (1+t)} \tau\left(T \chi_{\left(\frac{1}{t}, \infty\right)}(T)\right)
$$

существует и равен, скажем, $B$, то

$$
\limsup _{t \rightarrow \infty} \frac{1}{\log (1+t)} \int_{0}^{t} \mu_{s}(T) d s \leqslant B \leqslant \alpha \liminf _{t \rightarrow \infty} \frac{1}{\log (1+t)} \int_{0}^{t} \mu_{s}(T) d s
$$

для всех $\alpha>1$ и поэтому также

$$
\lim _{t \rightarrow \infty} \frac{1}{\log (1+t)} \int_{0}^{t} \mu_{s}(T) d s=B
$$

Оставшиеся утверждения выводятся подобным образом.

10.2. Дзета-функция и след Диксмье. Стимулом для этого пункта служит предложение IV.2.4 из работы [7]. Мы опишем несколько обобщений этого результата на случай алгебр фон Неймана. Наш подход будет аналогичен подходу книги [7], хотя и несколько отличен от него.

Дзета-функция положительного оператора $T \in \mathscr{L}^{(1, \infty)}$ задается формулой $\zeta(s)=\tau\left(T^{s}\right)$, в то время как для $A \in \mathscr{N}$ мы полагаем $\zeta_{A}(s)=\tau\left(A T^{s}\right)$. Мы интересуемся асимптотическим поведением функций $\zeta(s)$ и $\zeta_{A}(s)$ при $s \rightarrow 1$.

В этой связи легко увидеть важность обсуждения сингулярных следов, поскольку, согласно лемме 10.1 , для некоторого $K>0$ и для любого $s>1$ мы имеем

$$
\tau\left(T^{s}\right)=\int_{0}^{\infty} \mu_{r}\left(T^{s}\right) d r=\int_{0}^{\infty} \mu_{r}(T)^{s} d r \leqslant \int_{0}^{\infty} \frac{K^{s}}{(1+r)^{s}} d r=\frac{K^{s}}{s-1} .
$$

Из этой оценки следует, что множество $\left\{(s-1) \tau\left(T^{s}\right): s>1\right\}$ ограничено. Теперь для ограниченного оператора $A$ имеем $\left|(s-1) \tau\left(A T^{s}\right)\right| \leqslant\|A\|(s-1) \tau\left(T^{s}\right)$, поэтому $(s-1) \tau\left(A T^{s}\right)$ также ограничено и, следовательно, для любого $\widetilde{\omega} \in$ $L_{\infty}(\mathbb{R})^{*}$, удовлетворяющего условиям (1), (2) и (3) теоремы 4.7 , существует предел

$$
\overbrace{r \rightarrow \infty} \frac{1}{r} \tau\left(A T^{1+\frac{1}{r}}\right) .
$$


Здесь функцию $r \rightarrow \frac{1}{r} \tau\left(A T^{1+\frac{1}{r}}\right)$ мы определяем как функцию на всем $\mathbb{R}$, продолжая ее тождественно равной нулю при $r<1$. Возможно, хотелось бы думать о выражении (19) как о $\widetilde{\omega-\lim }_{s \rightarrow 1}(s-1) \tau\left(A T^{s}\right)$, но это выражение, конечно, не имеет (строго говоря) смысла, тогда как если $\lim _{s \rightarrow 1}(s-1) \tau\left(A T^{s}\right)$ существует, то он совпадает с $\lim _{r \rightarrow \infty} \frac{1}{r} \tau\left(A T^{1+\frac{1}{r}}\right)$.

В следующей теореме мы возьмем положительный оператор $T \in \mathscr{L}^{(1, \infty)}$, $\|T\| \leqslant 1$, со спектральным разложением $T=\int \lambda d E(\lambda)$. Мы хотели бы интегрировать по $d \tau(E(\lambda))$; к сожалению, эти скаляры $\tau(E(\lambda))$, вообще говоря, бесконечны. Чтобы исправить это положение, мы вместо этого должны интегрировать по возрастающей (отрицательной) вещественнозначной функции $N_{T}(\lambda)=\tau(E(\lambda)-1)$ при $\lambda>0$. Вне 0 приращения $\tau(\triangle E(\lambda))$ и $\triangle N_{T}(\lambda)$, конечно же, тождественно равны.

Теорема 10.6. Для положительного оператора $T \in \mathscr{L}^{(1, \infty)},\|T\| \leqslant 1, u$ состояния $\widetilde{\omega} \in L_{\infty}(\mathbb{R})^{*}$, удовлетворяющего всем условиям теоремы 4.7 , положим $\widetilde{\omega}=\omega \circ L$, где $L$ задан в разделе 4 (перед определением 4.4). Тогда мы имеем:

$$
\tau_{\omega}(T)=\overbrace{\vec{\omega}-\lim } \frac{1}{r} \tau\left(T^{1+\frac{1}{r}}\right) .
$$

Если существует $\lim _{r \rightarrow \infty} \frac{1}{r} \tau\left(T^{1+\frac{1}{r}}\right)$, mo

$$
\tau_{\omega}(T)=\lim _{r \rightarrow \infty} \frac{1}{r} \tau\left(T^{1+\frac{1}{r}}\right)
$$

для произвольного инвариантного при растяжениях функционала $\omega \in$ $L_{\infty}\left(\mathbb{R}_{+}^{*}\right)^{*}$.

ДокАЗАТЕЛЬСтво. Согласно (19), мы можем применить *-слабую теорему Караматы к функции $\frac{1}{r} \tau\left(T^{1+\frac{1}{r}}\right)$. Сначала запишем

$$
\tau\left(T^{1+\frac{1}{r}}\right)=\int_{0^{+}}^{1} \lambda^{1+\frac{1}{r}} d N_{T}(\lambda)
$$

Таким образом, полагая $\lambda=e^{-u}$, получим

$$
\tau\left(T^{1+\frac{1}{r}}\right)=\int_{0}^{\infty} e^{-\frac{u}{r}} d \beta(u)
$$

где $\beta(u)=\int_{u}^{0} e^{-v} d N_{T}\left(e^{-v}\right)=-\int_{0}^{u} e^{-v} d N_{T}\left(e^{-v}\right)$. Поскольку замена переменных $\lambda=e^{-u}$ является строго убывающей, $\beta$ является на самом деле неотрицательной и возрастающей. Согласно *-слабой теореме Караматы, примененной к $\widetilde{\omega} \in L_{\infty}(\mathbb{R})^{*}$,

$$
\widetilde{\omega-\lim }_{r \rightarrow \infty} \frac{1}{r} \tau\left(T^{1+\frac{1}{r}}\right)=\widetilde{\omega-\lim } \frac{\beta(u)}{u} .
$$

Далее, подставляя $\rho=e^{-v}$, мы получаем:

$$
\widetilde{\omega-\lim } \frac{\beta(u)}{u}=\widetilde{\omega-\lim } \frac{1}{u} \int_{e^{-u}}^{1} \rho d N_{T}(\rho) .
$$


Положим $f(u)=\beta(u) / u$. Мы хотим сделать замену переменной $u=\log t$ или, другими словами, рассмотреть функцию $f \circ \log =L f$. Мы воспользуемся обсуждением, приведенным в разделе 4, которое говорит нам, что если начать с $M$-инвариантного функционала $\omega \in L_{\infty}\left(\mathbb{R}_{+}^{*}\right)^{*}$, то функционал $\widetilde{\omega}=\omega \circ L$ будет $H$-инвариантным, как и требуется в теореме. Затем мы имеем

$$
\begin{aligned}
\widetilde{\omega-\lim _{r \rightarrow \infty}} \frac{1}{r} \tau\left(T^{1+\frac{1}{r}}\right) & =\widetilde{\omega-\lim _{u \rightarrow \infty}} \frac{\beta(u)}{u}=\widetilde{\omega-\lim _{u \rightarrow \infty}} f(u) \\
& =\underset{t \rightarrow \infty}{\omega-\lim _{t \rightarrow \infty}} L f(t)={\underset{t \rightarrow \infty}{\omega-\lim }}_{t} \frac{1}{\log t} \int_{1 / t}^{1} \lambda d N_{T}(\lambda) .
\end{aligned}
$$

Теперь по предложению 10.5

$$
\underset{t \rightarrow \infty}{\omega-\lim } \frac{1}{\log t} \int_{1 / t}^{1} \lambda d N_{T}(\lambda)=\underset{t \rightarrow \infty}{\omega} \lim \frac{1}{\log t} \tau\left(\chi_{\left(\frac{1}{t}, 1\right]}(T) T\right)=\tau_{\omega}(T) .
$$

Это завершает доказательство первой части теоремы.

Доказательство второй части похоже. Используя классическую теорему Караматы (см. замечание 10.3), мы получим следующий аналог равенства (20):

$$
\lim _{r \rightarrow \infty} \frac{1}{r} \tau\left(T^{1+r}\right)=\lim _{u \rightarrow \infty} \frac{\beta(u)}{u}=\lim _{u \rightarrow \infty} \frac{1}{u} \int_{e^{-u}}^{1} \rho d N_{T}(\rho) .
$$

Делая подстановку $u=\log t$ в правой части, мы имеем

$$
\lim _{u \rightarrow \infty} \frac{1}{u} \int_{e^{-u}}^{1} \rho d N_{T}(\rho)=\lim _{t \rightarrow \infty} \frac{1}{\log t} \int_{\frac{1}{t}}^{1} \lambda d N_{T}(\lambda)=\tau_{\omega}(T),
$$

где в последнем равенстве нам необходима только инвариантность состояния $\omega \in L_{\infty}\left(\mathbb{R}_{+}^{*}\right)^{*}$ при растяжениях, а не весь список условий следствия 4.8. Теорема 10.6 доказана.

Отображение со значениями в $\mathbb{R}$, определенное на положительных операто$\operatorname{pax} T \in \mathscr{L}^{(1, \infty)}$ по формуле $T \rightarrow \tau_{\omega}(T)$, может быть продолжено по линейности до $\mathbb{C}$-значного функционала на всем $\mathscr{L}^{(1, \infty)}$. Тогда корректно определен функционал

$$
A \mapsto \tau_{\omega}(A T)
$$

для $A \in \mathscr{N}$ и фиксированного $T \in \mathscr{L}^{(1, \infty)}$. Мы намерены изучать свойства функционала (21). Частично, интерес к этому функционалу происходит из следующего результата (см. [61]), а также из применений следа Диксмье в некоммутативной геометрии [7].

Лемма 10.7. Пусть $T \in \mathscr{L}^{(1, \infty)}$. Тогда справедливы следующие утверждения.

(i) Для $A \in \mathscr{N}$ имеем

$$
\tau_{\omega}(A T)=\tau_{\omega}(T A) .
$$

(ii) Предположим, что $D_{0}$ - такой неограниченный самосопряженный оператор, присоединенный $\kappa \mathscr{N}$, что $T=\left(1+D_{0}^{2}\right)^{-1 / 2} \in \mathscr{L}^{(1, \infty)}$. Eсли $\left[A_{j},\left|D_{0}\right|\right]$ является ограниченным оператором при $A_{j} \in \mathscr{N}, j=1,2$, то

$$
\tau_{\omega}\left(A_{1} A_{2} T\right)=\tau_{\omega}\left(A_{2} A_{1} T\right) .
$$


Доказательство. (i) Это утверждение есть не что иное, как предложение А.2 в работе [85]. Доказательство элементарно: сначала покажем, что $\tau_{\omega}\left(U T U^{*}\right)=\tau_{\omega}(T)$, затем, используя линейность, продолжим это равенство на произвольные $T \in \mathscr{L}^{(1, \infty)}$. Заменим $T$ на $T U$, затем снова используем линейность.

(ii) Заметим, что утверждение о том, что $\left[A_{j},\left|D_{0}\right|\right]$ определяет ограниченный оператор, означает, что оператор $A_{j}$ переводит $\operatorname{dom}\left(\left|D_{0}\right|\right)=\operatorname{dom}\left(D_{0}\right)$ в себя и что оператор $\left[A_{j},\left|D_{0}\right|\right]$ ограничен на этой области определения (см. [86; предложение 3.2.55] и его доказательство по поводу эквивалентных, но кажущихся более слабыми условий $)$. Поскольку $\left|D_{0}\right|-\left(1+D_{0}^{2}\right)^{1 / 2}$ ограничен, $\left[A_{j},\left(1+D_{0}^{2}\right)^{1 / 2}\right]$ определяет ограниченный оператор, если то же самое верно для $\left[A_{j},\left|D_{0}\right|\right]$. Так как $T^{-1}=\left(1+D_{0}^{2}\right)^{1 / 2}$ и $T: \mathscr{H} \rightarrow \operatorname{dom}\left(T^{-1}\right)$, мы видим, что формальное равенство

$$
\left[A_{j}, T\right]=A_{j} T-T A_{j}=T\left(T^{-1} A_{j}-A_{j} T^{-1}\right) T=T\left[T^{-1}, A_{j}\right] T
$$

имеет смысл как равенство всюду определенных ограниченных операторов в $\mathscr{H}$. То есть

$$
\left[A_{j}, T\right]=T\left[\left(1+D_{0}^{2}\right)^{1 / 2}, A_{j}\right] T \in\left(\mathscr{L}^{(1, \infty)}\right)^{2} \subseteq \mathscr{L}^{1} .
$$

Тогда, используя часть (i), мы имеем

$$
\tau_{\omega}\left(A_{1} A_{2} T\right)=\tau_{\omega}\left(A_{2} A_{1} T\right)-\tau_{\omega}\left(\left[A_{1}, T\right] A_{2}\right) .
$$

Поэтому

$$
\tau_{\omega}\left(A_{1} A_{2} T\right)=\tau_{\omega}\left(A_{2} A_{1} T\right)-\tau_{\omega}\left(T\left[\left(1+D_{0}^{2}\right)^{1 / 2}, A_{1}\right] T A_{2}\right) .
$$

Поскольку оператор, стоящий в последнем слагаемом, является ядерным, доказательство закончено.

Мы будем рассматривать спектральные тройки, что включает в себя выбор подалгебры в $\mathscr{N}$, на которой функционал (21) будет определять след.

TeOpema 10.8. Пусть $A \in \mathscr{N}, T \geqslant 0, T \in \mathscr{L}^{(1, \infty)}$.

(i) Если существует предел $\lim _{s \rightarrow 1^{+}}(s-1) \tau\left(A T^{s}\right)$, то он равен $\tau_{\omega}(A T)$, где $\omega$ выбирается как в доказательстве теоремы 10.6.

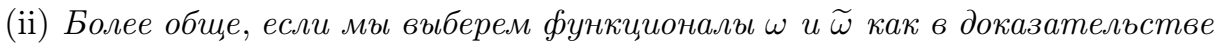
теоремы 10.6, то

$$
\varlimsup_{r \rightarrow \infty} \frac{1}{r} \tau\left(A T^{1+\frac{1}{r}}\right)=\tau_{\omega}(A T) .
$$

ДокАЗАТЕльство. Для доказательства части (i) мы сначала предположим, что $A$ самосопряжен. Запишем $A=a^{+}-a^{-}$, где $a^{ \pm}$положительны. Выберем $\widetilde{\omega}$ как в доказательстве теоремы 10.6. Тогда

$$
\begin{aligned}
\lim _{s \rightarrow 1^{+}}(s-1) \tau\left(A T^{s}\right) & =\widetilde{\omega-\lim _{r \rightarrow \infty}} \frac{1}{r} \tau\left(A T^{1+\frac{1}{r}}\right) \\
& =\widetilde{\omega-\lim _{r \rightarrow \infty}} \frac{1}{r} \tau\left(a^{+} T^{1+\frac{1}{r}}\right)-\widetilde{\omega-\lim _{r \rightarrow \infty}} \frac{1}{r} \tau\left(a^{-} T^{1+\frac{1}{r}}\right) \\
& =\tau_{\omega}\left(a^{+} T\right)-\tau_{\omega}\left(a^{-} T\right)=\tau_{\omega}(A T) .
\end{aligned}
$$


Здесь третье равенство использует технический результат (предложение 3.6) из работы [29] и затем теорему 10.6. Редукция общего случая к самосопряженному случаю проводится теперь подобным образом.

Для доказательства части (ii) мы предположим, что $A$ положителен. Из леммы 10.7(i), теоремы 10.6 и [29; предложение 3.6] мы имеем

$$
\tau_{\omega}(A T)=\tau_{\omega}\left(A^{1 / 2} T A^{1 / 2}\right)=\widetilde{\omega-\lim _{r \rightarrow \infty}} \frac{1}{r} \tau\left(\left(A^{1 / 2} T A^{1 / 2}\right)^{1+\frac{1}{r}}\right)=\widetilde{\omega-\lim } \frac{1}{r} \tau\left(A T^{1+\frac{1}{r}}\right) .
$$

Для общего случая мы сводим рассмотрения к случаю, когда $A$ положителен, как в доказательстве части (i).

\section{1. Формула в терминах уравнения теплопроводности}

Содержание этого раздела следует идеям [7] и снова взято из работы [29]. Мы сохраним повсюду предположение о том, что $T \geqslant 0$, и определим $e^{-T^{-2}}$ как оператор, который равен нулю на $\operatorname{ker} T$, a на $\operatorname{ker} T^{\perp}$ определен при помощи функционального исчисления. Заметим, что если $T \geqslant 0$ и $T \in \mathscr{L}^{(p, \infty)}$ при некотором $p \geqslant 1$, то оператор $e^{-t T^{-2}}$ является ядерным для любого $t>0$.

Нашей целью в этом разделе является доказательство следующей теоремы.

Tеорема 11.1. Если $A \in \mathscr{N}, T \geqslant 0, T \in \mathscr{L}^{(1, \infty)}$, mo

$$
\underset{\lambda \rightarrow \infty}{\omega} \lim ^{-1} \tau\left(A e^{-\lambda^{-2} T^{-2}}\right)=\Gamma(3 / 2) \tau_{\omega}(A T)
$$

для $\omega \in L_{\infty}\left(\mathbb{R}_{+}^{*}\right)^{*}$, удовлетворяющего условиям следствия 4.8 .

Пусть $\zeta_{A}\left(p+\frac{1}{r}\right)=\tau\left(A T^{p+\frac{1}{r}}\right)$. Заметим, что всегда существует предел

$$
\frac{1}{2} \Gamma\left(\frac{p}{2}\right) \underset{r \rightarrow \infty}{\omega-\lim } \frac{1}{r} \zeta_{A}\left(p+\frac{1}{r}\right) .
$$

Следовательно, мы можем свести трудную часть доказательства теоремы 11.1 к следующему предварительному результату.

ПредлОЖение 11.2. Если $A \in \mathscr{N}, A \geqslant 0, T \geqslant 0, T \in \mathscr{L}^{(p, \infty)}, 1 \leqslant p<\infty$,

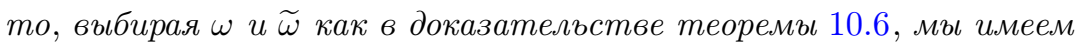

$$
\underset{\lambda \rightarrow \infty}{\omega-\lim _{\lambda}} \frac{1}{\lambda} \tau\left(A e^{-T^{-2} \lambda^{-2 / p}}\right)=\frac{1}{2} \Gamma\left(\frac{p}{2}\right) \widetilde{\omega-\lim _{r \rightarrow \infty}} \frac{1}{r} \zeta_{A}\left(p+\frac{1}{r}\right) .
$$

ДокАзАТЕльство. Используя преобразование Лапласа, мы имеем

$$
T^{s}=\frac{1}{\Gamma(s / 2)} \int_{0}^{\infty} t^{s / 2-1} e^{-t T^{-2}} d t
$$

Тогда

$$
\zeta_{A}(s)=\tau\left(A T^{s}\right)=\frac{1}{\Gamma(s / 2)} \int_{0}^{\infty} t^{s / 2-1} \tau\left(A e^{-t T^{-2}}\right) d t .
$$

Сделаем замену переменных $t=1 / \lambda^{2 / p}$, так что предыдущая формула примет вид

$$
\frac{p}{2} \Gamma(s / 2) \zeta_{A}(s)=\int_{0}^{\infty} \lambda^{-\frac{s}{p}-1} \tau\left(A e^{-\lambda^{-2 / p} T^{-2}}\right) d \lambda .
$$


Мы разобьем этот интеграл на две части, $\int_{0}^{1}$ и $\int_{1}^{\infty}$, и обозначим первый интеграл $R(r)$, где $s=p+\frac{1}{r}$. Тогда

$$
R(r)=\int_{0}^{1} \lambda^{-\frac{1}{p r}-2} \tau\left(A e^{-\lambda^{-2 / p} T^{-2}}\right) d \lambda=\int_{1}^{\infty} t^{\frac{p}{2}+\frac{1}{2 r}-1} \tau\left(A e^{-t T^{-2}}\right) d t .
$$

Подынтегральное выражение убывает экспоненциально по $t$ при $t \rightarrow \infty$, поскольку $T^{-2} \geqslant\left\|T^{2}\right\|^{-1} \mathbf{1}$. Тем самым,

$$
\tau\left(A e^{-t T^{-2}}\right) \leqslant \tau\left(A e^{-T^{-2}} e^{-\frac{t-1}{\left\|T^{2}\right\|}}\right) .
$$

Значит, можно заключить, что $R(r)$ ограничен равномерно по $r$ и, тем самым, $\lim _{r \rightarrow \infty} \frac{1}{r} R(r)=0$. Для другого интеграла замена переменных $\lambda=e^{\mu}$ дает

$$
\int_{1}^{\infty} \lambda^{-\frac{1}{p r}-2} \tau\left(A e^{-\lambda^{-2 / p} T^{-2}}\right) d \lambda=\int_{0}^{\infty} e^{-\frac{\mu}{p r}} d \beta(\mu)
$$

где

$$
\beta(\mu)=\int_{0}^{\mu} e^{-v} \tau\left(A e^{-e^{-\frac{2}{p} v} T^{-2}}\right) d v .
$$

Следовательно, сейчас мы можем написать

$$
\frac{p}{2} \Gamma\left(\left(p+\frac{1}{r}\right) / 2\right) \zeta_{A}\left(p+\frac{1}{r}\right)=\int_{0}^{\infty} e^{-\frac{\mu}{p r}} d \beta(\mu)+R(r) .
$$

Теперь рассмотрим

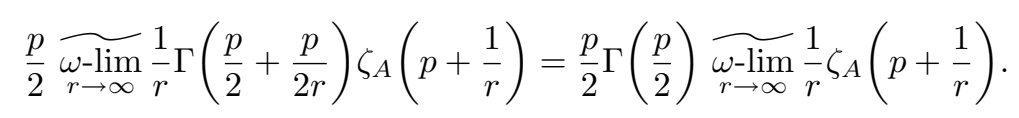

Тогда

$$
\frac{p}{2} \Gamma\left(\frac{p}{2}\right) \widetilde{\omega-\lim } \frac{1}{r} \zeta_{A}\left(p+\frac{1}{r}\right)=p \widetilde{\omega-\lim } \frac{1}{p r} \int_{0}^{\infty} e^{-\frac{\mu}{p r}} d \beta(\mu)
$$

(напомним, что член $\frac{1}{r} R(r)$ имеет нулевой предел при $r \rightarrow \infty$ ). Используя инвариантность при растяжениях и теорему 10.2 , мы имеем

$$
\frac{p}{2} \Gamma\left(\frac{p}{2}\right) \widetilde{\omega-\lim } \frac{1}{r} \zeta_{A}\left(p+\frac{1}{r}\right)=p \underset{\omega-\lim }{\widetilde{\beta}} \frac{\beta(\mu)}{\mu} .
$$

Делая замену переменных $\lambda=e^{v}$ в выражении для $\beta(\mu)$, мы получаем

$$
\frac{\beta(\mu)}{\mu}=\frac{1}{\mu} \int_{1}^{e^{\mu}} \lambda^{-2} \tau\left(A e^{-T^{-2} \lambda^{-2 / p}}\right) d \lambda .
$$

После подстановки $\mu=\log t$ правая часть этого равенства примет вид

$$
\frac{1}{\log t} \int_{1}^{t} \lambda^{-2} \tau\left(A e^{-T^{-2} \lambda^{-2 / p}}\right) d \lambda=g_{1}(t),
$$

что совпадает со средним по Чезаро от функции

$$
g_{2}(\lambda)=\frac{1}{\lambda} \tau\left(A e^{-T^{-2} \lambda^{-2 / p}}\right) .
$$


Тогда, поскольку мы выбрали $\omega \in L_{\infty}\left(\mathbb{R}_{+}^{*}\right)^{*}$ удовлетворяющим условиям следствия 4.8, мы имеем $\omega\left(g_{1}\right)=\omega\left(g_{2}\right)$. Вспоминая, что мы выбрали $\widetilde{\omega}$ связанным с $\omega$ как в теореме 10.6 и поэтому можно использовать (22), мы получаем

$$
\underset{\lambda \rightarrow \infty}{\omega-\lim _{\lambda}} \frac{1}{\lambda} \tau\left(A e^{-T^{-2} \lambda^{-2 / p}}\right)=\frac{1}{2} \Gamma\left(\frac{p}{2}\right) \widetilde{\omega-\lim _{r \rightarrow \infty}} \frac{1}{r} \zeta_{A}\left(p+\frac{1}{r}\right) .
$$

Предложение 11.2 доказано.

Для доказательства теоремы мы сначала рассмотрим случай, когда $A$ ограничен, $A \geqslant 0$, и применим предложение 11.2 и теорему 10.8 , чтобы получить, что

$$
\Gamma\left(\frac{3}{2}\right) \tau_{\omega}(A T)=\Gamma\left(\frac{3}{2}\right) \widetilde{\omega-\lim _{r \rightarrow \infty}} \frac{1}{r} \tau\left(A T^{1+\frac{1}{r}}\right)=\underset{\lambda \rightarrow \infty}{\omega-\lim _{\lambda}} \lambda^{-1} \tau\left(A e^{-\lambda^{-2} T^{-2}}\right) .
$$

Затем для самосопряженного оператора $A$ запишем $A=a^{+}-a^{-}$, где $a^{ \pm}$положительны, так что

$$
\begin{aligned}
& \Gamma(3 / 2) \tau_{\omega}(A T)=\Gamma(3 / 2)\left(\tau_{\omega}\left(a^{+} T\right)-\tau_{\omega}\left(a^{-} T\right)\right) \\
& =\underset{\lambda \rightarrow \infty}{\omega} \lim ^{-1} \tau\left(a^{+} e^{-\lambda^{-2} T^{-2}}\right)-\underset{\lambda \rightarrow \infty}{\omega-\lim } \lambda^{-1} \tau\left(a^{-} e^{-\lambda^{-2} T^{-2}}\right) \\
& =\underset{\lambda \rightarrow \infty}{\omega} \lim ^{-1} \tau\left(A e^{-\lambda^{-2} T^{-2}}\right) .
\end{aligned}
$$

Подобными рассуждениями можно распространить этот факт на произвольный ограниченный оператор $A$.

\section{2. Случай $p>1$}

Результаты предыдущих разделов имеют аналоги при $p>1$. Некоторые рассуждения в этом случае проще благодаря тому факту, что сингулярные числа для оператора $T \in \mathscr{L}^{(p, \infty)}$ при $p>1, T \geqslant 0$ удовлетворяют асимптотической оценке $\mu_{s}(T)=O\left(1 / s^{1 / p}\right)$. Более того,

$$
\tau\left(T^{p+\frac{1}{r}}\right)=\int_{0}^{1} \lambda^{p+1 / r} d N_{T}(\lambda)
$$

где $N_{T}(\lambda)=\tau(E(\lambda)-1)$ при $\lambda>0$ и $T=\int \lambda d E(\lambda)-$ спектральное разложение для $T$.

Теперь мы установим некоторые $\mathscr{L}^{(p, \infty)}$-версии наших предыдущих результатов.

Лемма 12.1. Для $T \in \mathscr{L}^{(p, \infty)} u \omega u \widetilde{\omega} \kappa а к$ в доказательстве теоремы 10.6 мъц имеем

$$
p \tau_{\omega}\left(T^{p}\right)=\widetilde{\omega-\lim } \frac{1}{r} \tau\left(T^{p+\frac{1}{r}}\right) .
$$

ДокАЗАТЕЛЬСтво. Положим $\lambda=e^{-u / p}$, так что

$$
\frac{1}{r} \tau\left(T^{p+\frac{1}{r}}\right)=p \frac{1}{p r} \int_{0}^{\infty} e^{-u /(r p)} d \beta(u),
$$


где $\beta(u)=\int_{0}^{u} e^{-v} d N_{T}\left(e^{-v / p}\right)$. Используя инвариантность при растяжениях, получаем:

$$
\widetilde{\omega-\lim _{r \rightarrow \infty}} \frac{1}{r} \tau\left(T^{p+\frac{1}{r}}\right)=\widetilde{p} \underset{r \rightarrow \infty}{\lim } \frac{1}{p r} \int_{0}^{1} e^{-u /(p r)} d \beta(u)=p \widetilde{\omega-\lim _{u \rightarrow \infty}} \frac{\beta(u)}{u}
$$

согласно *-слабой теореме Караматы 10.2. Рассуждая, как в доказательстве теоремы 10.6 , и делая подстановку $\lambda=e^{-v / p}$ и $u=\log t$, мы имеем

$$
\begin{aligned}
\widetilde{\omega-\lim } \frac{\beta(u)}{u} & =\underset{t \rightarrow \infty}{\omega}-\lim _{t \rightarrow \infty} \frac{1}{\log t} \int_{t^{-1 / p}}^{1} \lambda^{p} d N_{T}(\lambda) \\
& =\underset{t \rightarrow \infty}{\omega}-\lim _{t \rightarrow \infty} \frac{1}{\log t} \tau\left(\chi_{\left(\frac{1}{t}, \infty\right)}\left(T^{p}\right) T^{p}\right)=\tau_{\omega}\left(T^{p}\right) .
\end{aligned}
$$

Лемма 12.1 доказана.

СлеДСтвиЕ 12.2. Пусть $T \geqslant 0, T \in \mathscr{L}^{(p, \infty)}$. Тогда

$$
\underset{\lambda \rightarrow \infty}{\omega-\lim _{\lambda}} \frac{1}{\lambda} \tau\left(e^{-T^{-2} \lambda^{-2 / p}}\right)=\Gamma\left(1+\frac{p}{2}\right) \underset{r \rightarrow \infty}{\omega-\lim } \frac{1}{r} \tau\left(T^{p+\frac{1}{r}}\right) .
$$

ДокАЗАТЕЛЬство. Достаточно применить предложение 11.2 и лемму 12.1.

$\mathscr{L}^{(p, \infty)}$-версия теоремы 10.8 и следующий результат Конна доказываются, следуя тем же методам, что и при $p=1$. Как описано в [29; раздел 5], необходимо несколько прямых обобщений разных технических результатов.

Tеорема 12.3. Если А ограничен, $T \geqslant 0$ и $T \in \mathscr{L}^{(p, \infty)}$ nри $p \geqslant 1$, mо

$$
\underset{\lambda \rightarrow \infty}{\omega} \lim ^{-1} \tau\left(A e^{-\lambda^{-2 / p} T^{-2}}\right)=\Gamma(1+p / 2) \tau_{\omega}\left(A T^{p}\right) .
$$

Наконец, теперь просто получить следующий результат.

Теорема 12.4. Если А ограничен, $T \geqslant 0, T \in \mathscr{L}^{(p, \infty)}$ и существует предел

$$
\lim _{s \rightarrow p^{+}}(s-p) \tau\left(A T^{s}\right),
$$

то он равен $р \tau_{\omega}\left(A T^{p}\right)$.

\section{3. Обобщенные тёплицевы операторы и их индекс}

Теория индекса тёплицевых операторов имеет длинную историю, которая отчасти берет начало в работах [87] и [88]. Мы объясним, как в этой теории можно использовать результаты, полученные в предыдущих разделах.

Как и прежде, $P$ обозначает проектор на неотрицательное спектральное подпространство неограниченного самосопряженного оператора $\mathscr{D}_{0}$ (имеющего ограниченный обратный), и мы интересуемся индексом Брейера-Фредгольма оператора $P u P$, действующего на $P \mathscr{H}$. Когда $\mathscr{N}=\mathscr{L}(\mathscr{H})$ и $\mathscr{D}_{0}-$ компонента $\mathscr{L}^{(1, \infty)}$-суммируемой спектральной тройки, наиболее общая теорема такого типа в данной ситуации алгебр типа $I$ получена в работе [85], где показано, что

$$
\operatorname{ind}(P u P)=\frac{1}{2} \tau_{\omega}\left(u\left[\mathscr{D}_{0}, u^{*}\right]\left|\mathscr{D}_{0}\right|^{-1}\right) .
$$


Сейчас мы покажем, что эта формула справедлива, когда $\mathscr{N}$ - общая полуконечная алгебра фон Неймана.

ЗАмечАниЕ. Конечно, работа [85] изучает намного более общую ситуацию, которая применима, например, в случае, когда $\mathscr{D}_{0}-$ компонента $\mathscr{L}^{(p, \infty)}$-суммируемой спектральной тройки. Авторы получили очень общую формулу для индекса оператора $P u P$ в терминах сумм вычетов дзета-функций, построенных по $\mathscr{D}_{0}$, при некоторых предположениях на аналитические свойства этих дзетафункций. Некоторые, но не все, из дзета-функций, входящих в их формулу, являются следами Диксмье. Мы не будем здесь пытаться описать этот результат, так как имеется прекрасный обзор в статье [89]. Теорема из [85] доказана в случае алгебр типа $I$, и ее обобщение на полуконечные алгебры фон Неймана выполнено в работах [25], [26].

Нам необходим следующий предварительный результат [29; лемма 6.1].

Лемма 13.1. Пусть $D_{0}$ - такой ограниченный самосопряженный оператор, присоединенный $\kappa \mathscr{N}$, что оператор $\left(1+D_{0}^{2}\right)^{-1 / 2}$ принадлежст $\mathscr{L}^{(1, \infty)}$. Пусть $A_{t}$ u $B$ принадлежат $\mathscr{N}$ при $t \in[0,1]$, причем $A_{t}$ самосопряжен $u$ отображсние $t \mapsto A_{t}$ непрерывно. Пусть $D_{t}=D_{0}+A_{t}, u$ пусть $p$ - вещественное число с $1<p<4 / 3$. Тогда величина

$$
\tau\left(B\left(1+D_{0}^{2}\right)^{-p / 2}-B\left(1+D_{t}^{2}\right)^{-p / 2}\right)
$$

равномерно ограничена по $t \in[0,1]$ u $p \in(1,4 / 3)$.

Tеорема 13.2. Пусть $\left(\mathscr{N}, \mathscr{D}_{0}\right)$ - $\mathscr{L}^{(1, \infty)}$-суммируемый модуль БрейераФредгольма для униталъной банаховой *-алгебры $\mathscr{A}$, и пусть и $\in \mathscr{A}$ - такой унитарный элемент, что $\left[\mathscr{D}_{0}, u\right]$ ограничен. Пусть $P$ - проектор на неотрищательное спектральное подпространство оператора $\mathscr{D}_{0}$. Тогда для $\omega$, въбранного как в следствии 4.8,

$$
\begin{aligned}
\operatorname{ind}(P u P) & =\operatorname{sf}\left(\mathscr{D}_{0}, u \mathscr{D}_{0} u^{*}\right)=\lim _{p \rightarrow 1^{+}} \frac{1}{2}(p-1) \tau\left(u\left[\mathscr{D}_{0}, u^{*}\right]\left(1+\mathscr{D}_{0}^{2}\right)^{-p / 2}\right) \\
& =\frac{1}{2} \tau_{\omega}\left(u\left[\mathscr{D}_{0}, u^{*}\right]\left(1+\mathscr{D}_{0}^{2}\right)^{-1 / 2}\right)=\frac{1}{2} \tau_{\omega}\left(u\left[\mathscr{D}_{0}, u^{*}\right]\left|\mathscr{D}_{0}\right|^{-1}\right),
\end{aligned}
$$

где последнее равенство справедливо только в том случае, когда $\mathscr{D}_{0}$ имеет ограниченный обратный.

Замечание 13.3. (1) Равенство

$$
\operatorname{ind}(P u P)=\frac{1}{2} \tau_{\omega}\left(u\left[\mathscr{D}_{0}, u^{*}\right]\left|\mathscr{D}_{0}\right|^{-1}\right)
$$

из теоремы 13.2 следует сравнить с теоремой IV.2.8 из [7]. В случае, когда $\mathscr{N}=\mathscr{L}(\mathscr{H})$, правая часть формулы $(23)$ является 1-коциклом Хохшильда на $\mathscr{A}$, который, как известно, равен характеру Черна $\mathscr{L}^{(1, \infty)}$-суммируемого фредгольмова модуля $\left(\mathscr{A}, \mathscr{D}_{0}, \mathscr{H}\right)$.

(2) Поскольку любой 1-суммируемый модуль, очевидно, является $\mathscr{L}^{(1, \infty)}$ _ суммируемым модулем, из теоремы следует, что любой неограниченный 1суммируемый модуль должен иметь тривиальное спаривание с $K_{1}(\mathscr{A})$ и поэтому не интересен с гомологической точки зрения. 
ДоказАтЕЛЬСтво. Пусть $\mathscr{D}_{t}^{u}=\mathscr{D}_{0}+t u\left[\mathscr{D}_{0}, u^{*}\right], t \in[0,1]$. Тогда, согласно [24; п. 2.1, (2)] мы имеем для любого $p>1$, что

$$
\operatorname{ind}(P u P)=\frac{1}{C_{p / 2}} \int_{0}^{1} \tau\left(u\left[\mathscr{D}_{0}, u^{*}\right]\left(1+\left(\mathscr{D}_{t}^{u}\right)^{2}\right)^{-p / 2}\right) d t .
$$

Теперь по лемме 13.1 величина

$$
\left|\tau\left(u\left[\mathscr{D}_{0}, u^{*}\right]\left[\left(1+\left(\mathscr{D}_{t}^{u}\right)^{2}\right)^{-p / 2}-\left(1+\mathscr{D}_{0}^{2}\right)^{-p / 2}\right]\right)\right|
$$

равномерно ограничена по $t$ и $p$ при $1<p<4 / 3$. Так как $\widetilde{C}_{p / 2} \rightarrow \infty$ при $p \rightarrow 1^{+}$, мы видим, что

$$
\begin{aligned}
\mid \operatorname{ind}(P u P)- & \frac{1}{C_{p / 2}} \tau\left(u\left[\mathscr{D}_{0}, u^{*}\right]\left(1+\mathscr{D}_{0}^{2}\right)^{-p / 2}\right) \mid \\
= & \mid \frac{1}{C_{p / 2}} \int_{0}^{1} \tau\left(u\left[\mathscr{D}_{0}, u^{*}\right]\left(1+\left(\mathscr{D}_{t}^{u}\right)^{2}\right)^{-p / 2}\right) d t \\
& \quad-\frac{1}{C_{p / 2}} \int_{0}^{1} \tau\left(u\left[\mathscr{D}_{0}, u^{*}\right]\left(1+\mathscr{D}_{0}^{2}\right)^{-p / 2}\right) d t \mid \\
\leqslant & \frac{1}{C_{p / 2}} \int_{0}^{1}\left|\tau\left(u\left[\mathscr{D}_{0}, u^{*}\right]\left[\left(1+\left(\mathscr{D}_{t}^{u}\right)^{2}\right)^{-p / 2}-\left(1+\mathscr{D}_{0}^{2}\right)^{-p / 2}\right]\right)\right| d t \\
\leqslant & \text { const } / C_{p / 2} \rightarrow 0 .
\end{aligned}
$$

Теперь элементарно, что при $p \rightarrow 1^{+}$

$$
\frac{2}{p-1}=\int_{|x| \geqslant 1}\left(\frac{1}{|x|}\right)^{p} d x \sim \int_{-\infty}^{\infty}\left(\frac{1}{\sqrt{1+x^{2}}}\right)^{p} d x=\widetilde{C}_{p / 2} .
$$

Это заканчивает доказательство первого равенства, в то время как второе равенство следует из теоремы 10.8 (i).

Третье равенство следует из равенства

$$
\left(\sqrt{1+\mathscr{D}_{0}^{2}}\right)^{-1}-\left|\mathscr{D}_{0}\right|^{-1}=\left(\sqrt{1+\mathscr{D}_{0}^{2}}\right)^{-1}\left|\mathscr{D}_{0}\right|^{-1}\left(\sqrt{1+\mathscr{D}_{0}^{2}}+\left|\mathscr{D}_{0}\right|\right)^{-1} .
$$

\section{4. Негладкие слоения и псевдодифференциальные операторы}

В этом разделе мы дадим дальнейшее приложение приведенных ранее результатов, следуя работам [22] и [36]. Основная цель диссертации Р. Принциса [36] состояла в том, чтобы установить формулу вычетов Водзицкого для следа Диксмье некоторых псевдодифференциальных операторов, ассоциированных с действием $\mathbb{R}^{n}$ на компактном пространстве $X$. Эти результаты были значительно обобщены в [22], однако подробное описание этой работы потребовало бы обсуждения теории слоений, поэтому мы ограничимся здесь кратким описанием простого случая, рассмотренного в [36].

Мы начнем с конструкции "группа-пространство с мерой" Мюррея-фон Неймана. Таким образом, $X$ - компактное пространство, наделенное вероятностной мерой $\nu$ и непрерывным свободным минимальным эргодическим действием $\alpha$ группы $\mathbb{R}^{n}$ на $X$, сохраняющим меру $\nu$. Мы будем записывать действие в виде $x \rightarrow t . x$ для $x \in X$ и $t \in \mathbb{R}^{n}$. Тогда скрещенное произведение $L_{\infty}(X, \nu) \times_{\alpha} \mathbb{R}^{n}$ есть фактор типа $I I$, содержащийся в ограниченных операторах на $L^{2}\left(\mathbb{R}^{n}, L^{2}(X, \nu)\right)$. Опишем эту конструкцию. Для функции $f \in$ 
$L^{1}\left(\mathbb{R}, L_{\infty}(X, \nu)\right) \subset L_{\infty}(X, \nu) \times_{\alpha} \mathbb{R}^{n}$ действие $f$ на векторе $\xi$ из $L^{2}\left(\mathbb{R}^{n}, L^{2}(X, \nu)\right)$ определяется скрученной левой сверткой следующим образом:

$$
(\widetilde{\pi}(f) \xi)(s)=\int_{\mathbb{R}^{n}} \alpha_{s}^{-1}(f(t)) \xi(s-t) d t .
$$

Здесь $f(t)$ - функция на $X$, действующая как оператор умножения в $L^{2}(X, \nu)$. Алгебра со скрученной сверткой

$$
L^{1}\left(\mathbb{R}^{n}, L_{\infty}(X, \nu)\right) \cap L^{2}\left(\mathbb{R}^{n}, L^{2}(X, \nu)\right)
$$

является плотным подпространством в $L^{2}\left(\mathbb{R}^{n}, L^{2}(X, \nu)\right)$, и на алгебре фон Неймана, которую она порождает, имеется канонический точный нормальный полуконечный след Tr. Эта алгебра фон Неймана есть

$$
\mathscr{N}=\left(\widetilde{\pi}\left(L_{\infty}(X, \nu) \times_{\alpha} \mathbb{R}^{n}\right)\right)^{\prime \prime} .
$$

Для функций $f, g: \mathbb{R}^{n} \rightarrow L_{\infty}(X)$, которые принадлежат $L^{2}\left(\mathbb{R}^{n}, L^{2}(X, \nu)\right)$ и чьи скрученные левые свертки $\widetilde{\pi}(f), \widetilde{\pi}(g)$ определяют ограниченные операторы на $L^{2}\left(\mathbb{R}^{n}, L^{2}(X, \nu)\right)$, след задается формулой

$$
\operatorname{Tr}\left(\tilde{\pi}(f)^{*} \tilde{\pi}(g)\right)=\int_{\mathbb{R}^{n}} \int_{X} f(t, x) g(t, x)^{*} d \nu(x) d t,
$$

где мы думаем о $f, g$ как о функциях на $\mathbb{R}^{n} \times X$.

Отождествим $L^{2}\left(\mathbb{R}^{n}\right)$ с $L^{2}\left(\mathbb{R}^{n}\right) \otimes 1 \subset L^{2}\left(\mathbb{R}, L^{2}(X, \nu)\right)$. Тогда любая скалярная функция $f$ на $\mathbb{R}^{n}$, которая является преобразованием Фурье $f=\widehat{g}$ ограниченной $L^{2}$-функции $g$, будет удовлетворять условию $f \in L^{2}\left(\mathbb{R}, L^{2}(X, \nu)\right)$ и $\tilde{\pi}(f)$ будет ограниченным оператором.

Псевдодифференциальные операторы определяются при помощи своих символов. Гладкий символ порядка $m$ есть такая функция $a: X \times \mathbb{R}^{n} \rightarrow \mathbb{C}$, что для любого $x \in X$ функция $a_{x}$, определяемая формулой $a_{x}(t, \xi)=a(t . x, \xi)$, удовлетворяет условиям

(1) $\sup \left\{\left|\partial_{\xi}^{\beta} \partial_{t}^{\gamma} a_{x}(t, \xi)(1+|\xi|)^{-m+|\beta|}:(t, \xi) \in \mathbb{R}^{n} \times \mathbb{R}^{n}, \beta, \gamma \in \mathbb{N}^{n},\right| \beta|+| \gamma \mid \leqslant\right.$ $M\}<\infty$ для всех $M \in \mathbb{N}$,

(2) $\xi \mapsto a_{x}(0, \xi)$ - гладкая функция на $\mathbb{R}^{n}$, прининающая значения в пространстве $\mathscr{C}^{\infty}(X)$ - множестве таких непрерывных функций $f$ на $X$, что функция $t \rightarrow(x \rightarrow f(t . x))$ является гладкой на $\mathbb{R}^{n}$.

Каждый символ $a$ определяет псевдодифференциальный оператор $\mathrm{Op}(a)$ на $C(X) \otimes C_{c}^{\infty}\left(\mathbb{R}^{n}\right)$ по формуле

$$
\mathrm{Op}(a) f(x, t)=\frac{1}{(2 \pi)^{n}} \int_{\mathbb{R}^{n}} e^{i t \xi} a(t . x, \xi) \widehat{f}(x, \xi) d \xi, \quad f \in C(X) \otimes C_{c}^{\infty}\left(\mathbb{R}^{n}\right),
$$

где $C_{c}^{\infty}\left(\mathbb{R}^{n}\right)$ - все гладкие функции на $\mathbb{R}^{n}$ с компактным носителем. Главный символ псевдодифференциального оператора $A$ на $X$ есть предел

$$
\sigma_{m}(A)(x, \xi)=\lim _{\lambda \rightarrow \infty} \frac{a(x, \lambda \xi)}{\lambda^{m}}, \quad(x, \xi) \in X \times \mathbb{R}^{n} \backslash\{0\},
$$

если он существует. Скажем, что $A$ эллиптичен, если его символ $a$ таков, что $a_{x}$ эллиптичен (т.е. обратим при всех достаточно больших $\xi$ ) для всех 
$x \in X$. Принцис изучает обратимые положительные эллиптические псевдодифференциальные операторы $A$, имеющие главный символ. В дальнейшем мы будем рассматривать только такие операторы. Дзета-функция такого оператора есть $\zeta(z)=\tau\left(A^{z}\right)$, и она существует, поскольку $A^{z}$ принадлежит идеалу $L_{1}(\mathscr{N}, \tau)[36]$ при $\operatorname{Re} z<-n / m$. Принцис показывает, что

$$
\lim _{x \rightarrow-\frac{n}{m}}\left(x+\frac{n}{m}\right) \zeta(x)=-\frac{1}{(2 \pi)^{n} m} \int_{X \times S^{n-1}} \sigma_{m}(A)(x, \xi)^{-\frac{n}{m}} d \nu(x) d \xi
$$

и что $A^{-\frac{n}{m}} \in \mathscr{L}^{(1, \infty)}(\mathscr{N}, \tau)$.

Теперь заметим, что из (24) вместе с теоремой 12.4 вытекает соотношение

$$
\tau_{\omega}\left(A^{-\frac{n}{m}}\right)=\frac{1}{(2 \pi)^{n} n} \int_{X \times S^{n-1}} \sigma_{m}(A)(x, \xi)^{-\frac{n}{m}} d \nu(x) d \xi .
$$

Другими словами, мы имеем вычет Водзицкого типа $I I$ для вычисления следа Диксмье таких псевдодифференциальных операторов.

Работа [22] рассматривает случай измеримых слоений в смысле работы [90]. Тем самым, слои слоения больше не задаются действием группы $\mathbb{R}^{n}$, как это было выше, а являются более общими подмногообразиями. Основной результат работы [22], относящийся к данной теме, заключается в формуле для следа Диксмье некоторых псевдодифференциальных операторов в терминах локального вычета, где последний является обобщением вычета Водзицкого в случае слоений.

\section{5. Алгебра почти периодических функций}

Этот раздел адаптирован из работы [79].

15.1. Почти периодические псевдодифференциальные операторы. Мы дадим обзор проведенных М. А. Шубиным [91] исследований теории индекса дифференциальных операторов с почти периодическими коэффициентами, которые развивают идеи работы [87]. Напомним, что тригонометрическая функция есть конечная линейная комбинация экспоненциальных функций $e_{\xi}: x \mapsto e^{i\langle x, \xi\rangle}$. Пространство $\operatorname{Trig}\left(\mathbb{R}^{n}\right)$ тригонометрических функций является *-подалгеброй $C^{*}$-алгебры $C_{b}\left(\mathbb{R}^{n}\right)$ непрерывных ограниченных функций. Равномерное замыкание пространства $\operatorname{Trig}\left(\mathbb{R}^{n}\right)$ называется алгеброй почти периодических функций и обозначается через $\mathscr{A} P\left(\mathbb{R}^{n}\right)$. Эта $C^{*}$-алгебра изоморфна алгебре непрерывных функций на $\mathbb{R}_{B}^{n}$ компактификации Бора пространства $\mathbb{R}^{n}$. Сложение в $\mathbb{R}^{n}$ продолжается на $\mathbb{R}_{B}^{n}$, которая является компактной абелевой группой, содержащей $\mathbb{R}^{n}$ как плотную подгруппу. Нормализованная мера Хаара $\alpha_{B}$ на $\mathbb{R}_{B}^{n}$ такова, что семейство $\left(e_{\xi}\right)_{\xi \in \mathbb{R}^{n}}$ ортонормировано. Мера $\alpha_{B}$ задается для любой почти периодической функции $f$ на $\mathbb{R}^{n}$ по формуле

$$
\alpha_{B}(f):=\lim _{T \rightarrow+\infty} \frac{1}{(2 T)^{n}} \int_{(-T, T)^{n}} f(x) d x .
$$

При помощи $\alpha_{B}$ определяется гильбертово пространство $L^{2}\left(\mathbb{R}_{B}^{n}\right)$, являющееся пополнением пространства $\operatorname{Trig}\left(\mathbb{R}^{n}\right)$. Это гильбертово пространство имеет 
ортонормированный базис, задаваемый функциями $\left(e_{\xi}\right)_{\xi \in \mathbb{R}^{n}}$. Преобразование Фурье $\mathscr{F}_{B}: \ell^{2}\left(\mathbb{R}_{d}^{n}\right) \rightarrow L^{2}\left(\mathbb{R}_{B}^{n}\right)$ задается формулой

$$
\mathscr{F}_{B}\left(\delta_{\xi}\right)=e_{\xi}, \quad \text { где } \delta_{\xi}(\eta)=\delta_{\xi, \eta} \quad \text { - символ Кронеккера. }
$$

Мы будем обозначать через $\mathscr{F}$ обычное преобразование Фурье на абелевой группе $\mathbb{R}^{n}$ с ее обычной мерой Лебега.

Действие $\mathbb{R}^{n}$ на $\mathbb{R}_{B}^{n}$ посредством сдвигов задает динамическую систему, причем естественно ассоциированная с ней алгебра фон Неймана есть скрещенное произведение $L_{\infty}\left(\mathbb{R}_{B}^{n}\right) \times \mathbb{R}^{n}$. Для приложений удобнее рассматривать коммутант этой алгебры фон Неймана, обозначая его через $\mathscr{N}$. Он также является скрещенным произведением, а именно, алгеброй фон Неймана $L_{\infty}\left(\mathbb{R}^{n}\right) \times \mathbb{R}_{d}^{n}$. Тем самым, $\mathscr{N}$ является фактором типа $I I_{\infty}$ с точным нормальным полуконечным следом $\tau$. Его можно описать как множество существенно ограниченных борелевских семейств $\left(A_{\mu}\right)_{\mu \in \mathbb{R}_{B}^{n}}$ ограниченных операторов в $L^{2}\left(\mathbb{R}^{n}\right)$, которые $\mathbb{R}^{n}$-эквивариантны, т.е. таковы, что

$$
A_{\mu}=\sigma_{\mu}\left(A_{0}\right)=T_{-\mu} A_{0} T_{\mu} \quad \forall \mu \in \mathbb{R}^{n} .
$$

Здесь и в дальнейшем мы обозначим через $\sigma_{\mu}$ сопряжение любого оператора со сдвигом $T_{\mu}$, так что $\sigma_{\mu}(B)=T_{-\mu} B T_{\mu}$. Если мы обозначим через $M_{\varphi}$ оператор умножения на ограниченную функцию $\varphi$, то примеры таких семейств даются для любого $\lambda$ семействами

$$
\left(\sigma_{\mu}\left(M_{e_{\lambda}}\right)\right)_{\mu \in \mathbb{R}_{B}^{n}}
$$

Мы выберем преобразование Фурье в виде

$$
\mathscr{F} f(\zeta)=\frac{1}{(2 \pi)^{n / 2}} \int_{\mathbb{R}^{n}} e^{i x \zeta} f(x) d x .
$$

Тогда алгебра фон Неймана $\mathscr{N}$ может быть определена [87] как пополнение в гильбертовом пространстве $\mathscr{H}=L^{2}\left(\mathbb{R}^{n}\right) \otimes L^{2}\left(\mathbb{R}_{B}^{n}\right)$ множества операторов $\left\{M_{e_{\lambda}} \otimes M_{e_{\lambda}}, T_{\lambda} \otimes 1\right\}$, где $\lambda$ изменяется в пространстве $\mathbb{R}^{n}$.

Можно естественным образом вложить $C^{*}$-алгебру $\mathscr{A} P\left(\mathbb{R}^{n}\right)$ в $\mathscr{N}$, полагая

$$
\pi(f):=\left(\sigma_{\mu}\left(M_{f}\right)\right)_{\mu \in \mathbb{R}_{B}^{n}} .
$$

Это семейство, тем самым, принадлежит $\mathscr{N}$, и ясно, что $\pi$ точное. Семейство $\pi(f)$, рассматриваемое как оператор в $\mathscr{H}$, задается формулой $\pi(f)(g)(x, \mu)=$ $f(x+\mu) g(x, \mu)$. Если $B=\left(B_{\mu}\right)_{\mu}$ - положительный элемент из $\mathscr{N}$, то мы определим условное ожидание $E(B)$ как интеграл Хаара:

$$
E(B):=\int_{\mathbb{R}_{B}^{n}} B_{\mu} d \alpha_{B}(\mu) .
$$

Поскольку семейство $B$ и мера $\alpha_{B}$ инвариантны при сдвигах, оператор $E(B)$, очевидно, коммутирует со сдвигами в $L^{2}\left(\mathbb{R}^{n}\right)$ и поэтому задается мультипликатором Фурье $\widetilde{M}\left(\varphi_{B}\right)$, где $\varphi_{B}$ - положительный элемент пространства $L^{\infty}\left(\mathbb{R}^{n}\right)$. Напомним, что мультипликатор Фурье $\widetilde{M}\left(\varphi_{B}\right)$ есть сопряжение оператора умножения $M_{\varphi}$ посредством преобразования Фурье, т.е. $\widetilde{M}\left(\varphi_{B}\right)=\mathscr{F}^{-1} M_{\varphi} \mathscr{F}$. Когда функция $\varphi$ принадлежит пространству Шварца, оператор $\widetilde{M}\left(\varphi_{B}\right)$ является оператором свертки с функцией из класса Шварца $\frac{1}{(2 \pi)^{n / 2}} \mathscr{F}^{-1} \varphi$. Следовательно, условное ожидание $E$ принимает значения в алгебре фон Неймана 
$\widetilde{M}\left(L^{\infty}\left(\mathbb{R}^{n}\right)\right)$, т.е.

$$
E: \mathscr{N} \rightarrow \widetilde{M}\left(L^{\infty}\left(\mathbb{R}^{n}\right)\right) .
$$

Теперь, используя обычный интеграл Лебега в $\mathbb{R}^{n}$ и нормировки работы [87], мы введем следующее определение следа $\tau$ :

$$
\tau(B)=\int_{\mathbb{R}^{n}} \varphi_{B}(\zeta) d \zeta
$$

ЛЕмма 15.1 [87]. Отображение $\tau$ является единственным с точностью до постоянной положительным нормальным, точным, полуконечным следом на $\mathscr{N}$.

Рассмотрим след на $\mathscr{N}$, определенный на операторах вида $M_{a} K$, где $a$ почти периодична и $K$ - оператор свертки в $L^{2}\left(\mathbb{R}^{n}\right)$, возникающий из умножения на $L^{1}$-функцию $k$ после применения преобразования Фурье. Имеем

$$
\tau\left(M_{a} K\right)=\lim _{T \rightarrow+\infty} \frac{1}{(2 T)^{n}} \int_{(-T, T)^{n}} a(x) d x \int_{\mathbb{R}^{n}} k(\zeta) d \zeta .
$$

Более обще, любой псевдодифференциальный оператор $A$ неположительного порядка $m$ в $L^{2}\left(\mathbb{R}^{n}, \mathbb{C}^{N}\right)$ с почти периодическими коэффициентами, действующий на $\mathbb{C}^{N}$-значных функциях, можно рассматривать как семейство над $\mathbb{R}_{B}^{n}$ псевдодифференциальных операторов в $\mathbb{R}^{n}$. Для этого начнем с того, что возьмем символ $a$ оператора $A$. Тогда оператор $\sigma_{\mu}(A)$ есть псевдодифференциальный оператор с почти периодическими коэффициентами, символ которого есть

$$
(x, \xi) \mapsto a(x+\mu, \xi) .
$$

Если $m \leqslant 0$, мы получим таким образом элемент алгебры $\mathscr{N}$. Обозначим через $\Psi_{A P}^{0}$ алгебру псевдодифференциальных операторов неположительного порядка с почти периодическими коэффициентами. Если порядок $m$ оператора $A$ строго больше 0 , то оператор $A^{\sharp}$, задаваемый семейством $\left(\sigma_{\mu}(A)\right)_{\mu \in \mathbb{R}_{B}^{n}}$, присоединен к $\mathscr{N}$. Если порядок $m$ оператора $A$ строго меньше $-n$, то ограниченный оператор $A^{\sharp}$ принадлежит идеалу $L_{1}$ по отношению к следу $\tau$ на алгебре фон Неймана $\mathscr{N} \otimes M_{N}(\mathbb{C})[92 ;$ предложение 3.3$]$ и мы имеем:

$$
\tau\left(A^{\sharp}\right)=\lim _{T \rightarrow+\infty} \frac{1}{(2 T)^{n}} \int_{(-T,+T)^{n} \times \mathbb{R}^{n}} \operatorname{Tr}(a(x, \zeta)) d x d \zeta .
$$

На самом деле, условное ожидание $E\left(A^{\sharp}\right)$ является псевдодифференциальным оператором на $\mathbb{R}^{n}$ с символом (обозначаемым через $E(a)$ и не зависящим от переменной $x$ ), который задается формулой

$$
E(a)(\zeta)=\lim _{T \rightarrow+\infty} \frac{1}{(2 T)^{n}} \int_{(-T,+T)^{n}} a(x, \zeta) d x .
$$

Следовательно, оператор $E\left(A^{\sharp}\right)$ в точности совпадает с мультипликатором Фурье $\widetilde{M}(E(a))$ и поэтому

$$
\tau\left(A^{\sharp}\right)=\int_{\mathbb{R}^{n}} \operatorname{Tr}(E(a)(\zeta)) d \zeta .
$$

Пусть $\Psi_{\mathrm{AP}}^{\infty}$ - пространство классических псевдодифференциальных операторов в $\mathbb{R}^{n}$ с почти периодическими коэффициентами. 
ТЕОРема 15.2. Пусть $A$ - (скалярный) псевдодифференциальный оператор c почти периодическими коэфбичиентами на $\mathbb{R}^{n}$. Предположим, что порядок $m$ оператора $A$ не превосходит $-n$, и обозначим через $a_{-n}$ часть символа а однородности $-n$. Тогда оператор $A^{\sharp}$ принадлежит идеалу Диксмье $L^{1, \infty}(\mathscr{N}, \tau)$. Более того, след Диксмъе $\tau_{\omega}\left(A^{\sharp}\right)$ оператора $A^{\sharp}$, ассоииированный с предельным прочессом $\omega$, не зависит от $\omega$ и задается формулой

$$
\tau_{\omega}\left(A^{\sharp}\right)=\frac{1}{n} \int_{\mathbb{R}_{B}^{n} \times \mathbb{S}^{n-1}} a_{-n}(x, \zeta) d \alpha_{B}(x) d \zeta .
$$

ДокАзАТЕЛЬство. Как обычно, обозначим через $\Delta$ оператор Лапласа в $\mathbb{R}^{n}$. Тогда оператор $A(1+\Delta)^{n / 2}$ является псевдодифференциальным оператором с почти периодическими коэффициентами и неположительным порядком. Следовательно, оператор $\left[A(1+\Delta)^{n / 2}\right]^{\sharp}=A^{\sharp}\left(1+\Delta^{\sharp}\right)^{n / 2}$ принадлежит алгебре фон Неймана $\mathscr{N}$. Далее, оператор $\left(1+\Delta^{\sharp}\right)^{-n / 2}$ является мультипликатором Фурье, определяемым функцией $\zeta \mapsto\left(1+\zeta^{2}\right)^{-n / 2}$. Поэтому если при $\lambda>0 \quad E_{\lambda}$ есть спектральный проектор оператора $(1+\Delta)^{-n / 2}$, соответствующий интервалу $(0, \lambda)$, то оператор $1-E_{\lambda}=\chi_{(\lambda,+\infty)}(1+\Delta)^{-n / 2}$ является мультипликатором Фурье, определяемым функцией $\zeta \mapsto f_{\lambda}\left(\left(\zeta^{2}+1\right)^{-n / 2}\right)$. Следовательно, след $\tau$ оператора $1-E_{\lambda}$ задается формулой

$$
\int_{\mathbb{R}^{n}} f_{\lambda}\left(\frac{1}{\left(\zeta^{2}+1\right)^{n / 2}}\right) d \zeta
$$

Легко вычислить этот интеграл и показать, что он пропорционален $1 / \lambda$. Тем самым, точная нижняя грань тех $\lambda$, для которых $\tau\left(1-E_{\lambda}\right) \leqslant t$, в точности пропорциональна $1 / t$. Следовательно, оператор $\left(1+\Delta^{\sharp}\right)^{-n / 2}$, а поэтому и оператор $A$ принадлежат идеалу Диксмье $L^{1, \infty}(\mathscr{N}, \tau)$.

Для вычисления следа Диксмье оператора $A$ мы применим теорему 10.1 из [91], чтобы вывести тот факт, что спектральная $\tau$-плотность $N_{A}(\lambda)$ оператора $A$ имеет асимптотическое разложение

$$
N_{A}(\lambda)=\frac{\gamma_{0}(A)}{\lambda}(1+o(1)), \quad \lambda \rightarrow+\infty,
$$

где $\gamma_{0}(A)$ задается формулой:

$$
\gamma_{0}(A)=\frac{1}{n} \int_{\mathbb{R}_{B}^{n} \times \mathbb{S} n-1} a_{-n}(x, \zeta) d \alpha_{B}(x) d \zeta .
$$

Теперь если $A$ положителен, то, согласно [22; предложение 1],

$$
\tau_{\omega}(A)=\lim _{\lambda \rightarrow+\infty} \lambda N_{A}(\lambda)=\gamma_{0}(A)
$$

Это доказывает теорему для положительного $A$. Поскольку отображение главного символа является гомоморфизмом, мы получаем результат для произвольного $A$. Теорема 15.2 доказана.

Нормировка, которую мы выбрали для следа в ситуации алгебр фон Неймана этого раздела, исключает множитель $1 /(2 \pi)^{n}$, который появляется в вычете Водзицкого в теории типа $I$. 
15.2. Почти периодическая спектральная тройка. Через $\mathscr{A}$ обозначим *-подалгебру алгебры $\mathscr{A} P^{\infty}\left(\mathbb{R}^{n}\right)$ гладких почти периодических функций на $\mathbb{R}^{n}$. В качестве гильбертова пространства, на котором действует алгебра $\mathscr{N}$, мы возьмем пространство $B^{2}\left(\mathbb{R}^{n}\right) \otimes L^{2}\left(\mathbb{R}^{n}\right)$, где $B^{2}\left(\mathbb{R}^{n}\right)$ - гильбертово пространство почти периодических функций на $\mathbb{R}^{n}$, в котором норма и скалярное произведение задаются ограничением на $\mathscr{A}$ следа Хаара на $\mathscr{A} P^{\infty}\left(\mathbb{R}^{n}\right)$ (заметим, что $\left.B^{2}\left(\mathbb{R}^{n}\right) \cong \ell^{2}\left(\mathbb{R}_{d}^{n}\right)\right)$. Эта алгебра фон Неймана типа $I I_{\infty}$ наделяется точным нормальным полуконечным следом, который мы обозначим через $\tau$. (Заметим, что явная формула для $\tau$ имеет такой же вид, как и в предыдущем пункте.)

Обычный оператор Дирака на $\mathbb{R}^{n}$ обозначается через $\mathscr{D}_{0}$. Тем самым, если $\mathscr{S}$ - спинорное представление $\mathbb{R}^{n}$, то $\mathscr{D}_{0}$ действует на гладких $\mathscr{S}$-значных функциях на $\mathbb{R}^{n}$. Оператор $\mathscr{D}_{0} \mathbb{Z}^{n}$-периодичен и присоединен к алгебре фон Неймана $\mathscr{N}_{\mathscr{S}}=\mathscr{N} \otimes \operatorname{End}(\mathscr{S})$. Последняя также является алгеброй фон Неймана типа $I I_{\infty}$, наделенной следом $\tau \otimes \operatorname{Tr}$. Более обще, для любого $N \geqslant 1$ мы будем обозначать через $\mathscr{N}_{\mathscr{S}, N}$ алгебру фон Неймана $\mathscr{N} \otimes \operatorname{End}\left(\mathscr{S} \otimes \mathbb{C}^{N}\right)$, наделенную следом $\tau \otimes \operatorname{Tr}$.

Алгебра $\mathscr{A}$ и ее замыкание точно представлены как $*$-подалгебры алгебры фон Неймана $\mathscr{N}_{\mathscr{S}}$. Таким же самым образом, алгебру $\mathscr{A} \otimes M_{N}(\mathbb{C})$ можно рассматривать как *-подалгебру алгебры $\mathscr{N}_{\mathscr{S}, N}$. Точнее, если $a \in \mathscr{A}$, то оператор $a^{\sharp}$, определяемый формулой

$$
\left(a^{\sharp} f\right)(x, y):=a(x+y) f(x, y) \quad \forall f \in B^{2}\left(\mathbb{R}^{n}\right) \otimes L^{2}\left(\mathbb{R}^{n}\right),
$$

принадлежит $\mathscr{N}_{S, N}$. Оператор $a^{\sharp}$ является в точности оператором, ассоциированным с дифференциальным оператором нулевого порядка, соответствующим умножению на $a$. Та же самая формула позволяет представить $\mathscr{A}$ в $\mathscr{N}_{\mathscr{S}}$.

ПредлОЖениЕ 15.3. Тройка $\left(\mathscr{A}, \mathscr{N}_{\mathscr{S}}, \mathscr{D}_{0}^{\sharp}\right)$ является полуконечной спектральной тройкой конечной размерности, равной $n$.

ДоказАтельство. Заметим, что алгебра $\mathscr{A}$ унитальна. Известно, что дифференциальный оператор $\mathscr{D}_{0}$ является эллиптическим, периодическим и самосопряженным на $\mathbb{R}^{n}$. Поэтому оператор $\mathscr{D}_{0}^{\sharp}$ присоединен к алгебре фон Неймана $\mathscr{N}_{\mathscr{S}}$, и он самосопряжен как плотно определенный неограниченный оператор на гильбертовом пространстве $B^{2}\left(\mathbb{R}^{n}\right) \otimes L^{2}\left(\mathbb{R}^{n}\right)$, причем $\mathscr{D}_{0}^{2}=\Delta \otimes \mathrm{Id}$, где $\Delta$ - обычный лапласиан. Для любой гладкой почти периодической функции $f$ на $\mathbb{R}^{n}$ коммутатор $\left[\mathscr{D}_{0}, f\right]$ является почти периодическим дифференциальным оператором 0-го порядка и, тем самым, $\left[\mathscr{D}_{0}^{\sharp}, f\right]$ принадлежит алгебре фон Неймана $\mathscr{N}$.

С другой стороны, псевдодифференциальный оператор $T=(\Delta+I)^{-1 / 2}$ является мультипликатором Фурье, ассоциированным с функцией

$$
k \mapsto \frac{1}{\left(\|k\|^{2}+1\right)^{1 / 2}} .
$$

Поэтому, как в доказательстве теоремы 15.2, можно вычислить явно его сингулярные числа $\mu_{t}(T)$ и показать, что они пропорциональны $t^{-1 / n}$. 


\section{6. Теорема Леша об индексе}

В этом разделе мы опишем (следуя работе [29]) доказательство теоремы об индексе, принадлежащей М. Лешу [88] (см. также [74]) для тёплицевых операторов с некоммутативными символами, которое опирается на подход к следу Диксмье, использующий дзета-функцию. Мы начнем с унитальной $C^{*}$-алгебры $\mathscr{A}$ с точным конечным следом $\tau_{\mathscr{A}}$, удовлетворяющим условию $\tau_{\mathscr{A}}(1)=1$, и непрерывного действия $\alpha$ группы $\mathbb{R}$ на $\mathscr{A}$, сохраняющего $\tau_{\mathscr{A}}$.

Обозначим через $H_{\tau_{\mathscr{A}}}$ гильбертово пополнение алгебры $\mathscr{A}$ по отношению к скалярному произведению $(a \mid b)=\tau\left(b^{*} a\right)$. Тем самым, $\mathscr{A}$ является гильбертовой алгеброй, и левое регулярное представление алгебры $\mathscr{A}$ на себе продолжается по непрерывности до представления $a \mapsto \pi_{\tau_{\mathscr{A}}}(a)$ алгебры $\mathscr{A}$ на $H_{\tau_{\mathscr{A}}}$ [93]. В дальнейшем мы будем опускать обозначение $\pi_{\tau_{\mathscr{A}}}$ и обозначать действие $\mathscr{A}$ на $H_{\tau_{\mathscr{A}}}$ при помощи размещения элементов друг рядом с другом.

Теперь мы обратимся к индуцированному представлению $\widetilde{\pi}$ скрещенного произведения $C^{*}$-алгебры $\mathscr{A} \times_{\alpha} \mathbb{R}$ на $L^{2}\left(\mathbb{R}, H_{\tau_{\mathscr{A}}}\right)$. То есть $\widetilde{\pi}$ есть представление $\pi \times \lambda$, получающееся из ковариантной пары $(\pi, \lambda)$ представлений системы $(\mathscr{A}, \mathbb{R}, \alpha)$, определяемых для $a \in \mathscr{A}, t, s \in \mathbb{R}$ и $\xi \in L^{2}\left(\mathbb{R}, H_{\tau_{\mathscr{A}}}\right)$ по формулам

$$
(\pi(a) \xi)(s)=\alpha_{s}^{-1}(a) \xi(s)
$$

и

$$
\lambda_{t}(\xi)(s)=\xi(s-t) .
$$

Тогда для функции $x \in L^{1}(\mathbb{R}, \mathscr{A}) \subset \mathscr{A} \times_{\alpha} \mathbb{R}$ действие элемента $\widetilde{\pi}(x)$ на векторе $\xi$ из $L^{2}\left(\mathbb{R}, H_{\tau_{\mathscr{A}}}\right)$ определяется следующим образом:

$$
(\widetilde{\pi}(x) \xi)(s)=\int_{-\infty}^{\infty} \alpha_{s}^{-1}(x(t)) \xi(s-t) d t .
$$

Теперь алгебра со скрученной сверткой $L^{1}(\mathbb{R}, \mathscr{A}) \cap L^{2}\left(\mathbb{R}, H_{\tau}\right)$ является плотным подпространством в $L^{2}\left(\mathbb{R}, H_{\tau}\right)$, а также гильбертовой алгеброй относительно заданного скалярного произведения. В таком случае имеется канонический точный, нормальный, полуконечный след, $\tau$, на алгебре фон Неймана

$$
\mathscr{N}=\left(\widetilde{\pi}\left(\mathscr{A} \times{ }_{\alpha} \mathbb{R}\right)\right)^{\prime \prime} .
$$

Для функций $x, y: \mathbb{R} \rightarrow \mathscr{A} \subset H_{\tau_{\mathscr{A}}}$, принадлежащих $L^{2}\left(\mathbb{R}, H_{\tau_{\mathscr{A}}}\right)$ и таких, что скрученные левые свертки $\widetilde{\pi}(x), \widetilde{\pi}(y)$ определяют ограниченные операторы в $L^{2}\left(\mathbb{R}, H_{\tau_{\mathscr{A}}}\right)$, этот след задается формулой

$$
\tau\left(\widetilde{\pi}(y)^{*} \widetilde{\pi}(x)\right)=:\langle x \mid y\rangle=\int_{-\infty}^{\infty} \tau_{\mathscr{A}}\left(x(t) y(t)^{*}\right) d t .
$$

В частности, если использовать отождествление $L^{2}(\mathbb{R})=L^{2}(\mathbb{R}) \otimes \chi_{\mathscr{A}} \subset$ $L^{2}\left(\mathbb{R}, H_{\tau_{\mathscr{A}}}\right)$, то любая скалярная функция $x$ на $\mathbb{R}$, являющаяся преобразованием Фурье $x=\widehat{f}$ ограниченной $L^{2}$-функции $f$, будет обладать свойствами: $x \in L^{2}\left(\mathbb{R}, H_{\tau_{\mathscr{A}}}\right)$ и $\widetilde{\pi}(x)$ - ограниченный оператор. Для таких скалярных функций $x$ оператор $\tilde{\pi}(x)$ есть в точности обычная свертка с функцией $x$ и обычно обозначается через $\lambda(x)$, поскольку это есть интегрированная форма представления $\lambda$. Следующая лемма легко получается из этих рассуждений. 
ЛЕмма 16.1. При обсуждавшихся выше предположениях и обозначениях

(i) если $h \in L^{2}(\mathbb{R})$, причем $\lambda(h)$ ограничен, и $a \in \mathscr{A}$, то, определяя $f: \mathbb{R} \rightarrow$ $H_{\tau}$ как $f(t)=a h(t)$, мы видим, что $f \in L^{2}\left(\mathbb{R}, H_{\tau_{\mathscr{A}}}\right) u \tilde{\pi}(f)=\pi(a) \lambda(h)$ ограничен,

(ii) если $g \in L^{1}(\mathbb{R}) \cap L^{\infty}(\mathbb{R})$ и $a \in \mathscr{A}$, то $\pi(a) \lambda(\widehat{g})$ принадлежит идеалу $L_{1}$ в $\mathscr{N} u$

$$
\operatorname{Tr}(\pi(a) \lambda(\widehat{g}))=\tau_{\mathscr{A}}(a) \int_{-\infty}^{\infty} g(t) d t .
$$

ДоказАТЕЛьство. Чтобы доказать часть $(\mathrm{i})$, возьмем $\xi \in C_{c}\left(\mathbb{R}, H_{\tau_{\mathscr{A}}}\right) \subseteq$ $L^{2}\left(\mathbb{R}, H_{\tau_{\mathscr{A}}}\right)$. Тогда

$$
\begin{aligned}
(\widetilde{\pi}(f) \xi)(s) & =\int_{-\infty}^{\infty} \alpha_{s}^{-1}(f(t)) \xi(s-t) d t=\int_{-\infty}^{\infty} \alpha_{s}^{-1}(a) h(t) \xi(s-t) d t \\
& =\alpha_{s}^{-1}(a) \int_{-\infty}^{\infty} h(t) \xi(s-t) d t=\alpha_{s}^{-1}(a)(\lambda(h) \xi)(s)=(\pi(a) \lambda(h) \xi)(s) .
\end{aligned}
$$

Чтобы доказать часть (ii), можно предположить, что функция $g$ неотрицательна и оператор а самосопряжен. Тогда положим $g=g^{1 / 2} g^{1 / 2}$, тем самым, $g^{1 / 2} \in L^{2} \cap L^{\infty}$ и поэтому $\lambda\left(\widehat{g^{1 / 2}}\right)$ ограничен. Теперь

$$
\pi(a) \lambda(\widehat{g})=\pi(a) \lambda\left(\widehat{g^{1 / 2}}\right) \pi\left(\chi_{\mathscr{A}}\right) \lambda\left(\widehat{g^{1 / 2}}\right)
$$

Тогда $\pi(a) \lambda\left(\widehat{g^{1 / 2}}\right)=\widetilde{\pi}(x)$, где $x(t)=a \widehat{g^{1 / 2}}(t)$, и $\pi\left(\chi_{\mathscr{A}}\right) \lambda\left(\widehat{g^{1 / 2}}\right)=\tilde{\pi}(y)$, где $y(t)=$ $\chi_{\mathscr{A}} \widehat{g^{1 / 2}}(t)$. Тем самым, $\widetilde{\pi}(x)$ и $\widetilde{\pi}(y)$ принадлежат $\mathscr{N}_{s a}$ и $\pi(a) \lambda(\widehat{g})=\tilde{\pi}(x) \widetilde{\pi}(y)$.

Следовательно,

$$
\begin{aligned}
\tau(\pi(a) \lambda(\widehat{g})) & =\tau(\widetilde{\pi}(x) \widetilde{\pi}(y))=\int_{-\infty}^{\infty} \tau(x(t) y(t)) d t \\
& =\tau_{\mathscr{A}}(a) \int_{-\infty}^{\infty}\left|\widehat{g^{1 / 2}}(t)\right|^{2} d t=\tau_{\mathscr{A}}(a) \int_{-\infty}^{\infty} g(s) d s
\end{aligned}
$$

Лемма 16.1 доказана.

По построению $\mathscr{N}$ является полуконечной алгеброй фон Неймана, наделенной точным, нормальным, полуконечным следом $\tau$ и точным представлением $\pi: \mathscr{A} \rightarrow \mathscr{N}$ [93]. Для каждого $t \in \mathbb{R} \quad \lambda_{t}$ является унитарным оператором в $U(\mathscr{N})$. Фактически, однопараметрическая унитарная группа $\left\{\lambda_{t}: t \in \mathbb{R}\right\}$ может быть записана как $\lambda_{t}=e^{i t \mathscr{D}}$, где $\mathscr{D}-$ неограниченный самосопряженный оператор

$$
\mathscr{D}=\frac{1}{2 \pi i} \frac{d}{d s}
$$

присоединенный к $\mathscr{N}$. При переходе к преобразованию Фурье (т.е. в спектральной картине для оператора $\mathscr{D})$, как в предыдущем предложении, оператор $\mathscr{D}$ становится умножением на независимую переменную и, тем самым, $f(\mathscr{D})$ становится поточечным умножением на функцию $f$. То есть

$$
\widetilde{\pi}(\widehat{f})=\lambda(\widehat{f})=f(\mathscr{D})
$$


И, следовательно, если $f$ - ограниченная $L^{1}$-функция, то

$$
\tau(f(\mathscr{D}))=\int_{-\infty}^{\infty} f(t) d t
$$

Согласно приведенным рассуждениям и предыдущей лемме, мы получаем следующий результат.

Лемма 16.2. Если $f \in L^{1}(\mathbb{R}) \cap L^{\infty}(\mathbb{R})$ и $a \in \mathscr{A}$, то $\pi(a) f(\mathscr{D})$ принадлежит идеалу $L^{1}$ в $\mathscr{N} u$

$$
\tau(\pi(a) f(\mathscr{D}))=\tau(a) \int_{-\infty}^{\infty} f(t) d t
$$

Пусть $\delta$ - плотно определенное (неограниченное) *-дифференцирование на $\mathscr{A}$, которое является инфинитезимальным генератором представления $\alpha: \mathbb{R} \rightarrow \operatorname{Aut}(\mathscr{A})$, и пусть $\widehat{\delta}-$ неограниченное *-дифференцирование на $\mathscr{N}$, которое является инфинитезимальным генератором представления $\operatorname{Ad} \circ \lambda: \mathbb{R} \rightarrow$ $\operatorname{Aut}(\mathscr{N})$ (здесь $\operatorname{Ad}\left(\lambda_{t}\right)$ обозначает сопряжение вида $\left.\lambda_{t}^{*} \cdot \lambda_{t}\right)$. Теперь, если $a \in \operatorname{dom}(\delta)$, то понятно, что $\pi(a) \in \operatorname{dom}(\widehat{\delta})$ и $\pi(\delta(a))=\widehat{\delta}(\pi(a))$. Согласно предложению 3.2 .55 в [86] (и его доказательству), $\pi(\delta(a))$ переводит область определения оператора $\mathscr{D}$ в себя и

$$
\pi(\delta(a))=2 \pi i[\mathscr{D}, \pi(a)]
$$

Теперь мы в состоянии сформулировать и доказать теорему Леша об индексе.

ТЕОРема 16.3 [29]. Пусть $\tau_{\mathscr{A}}-$ точный конечный след на униталъной $C^{*}$ алгебре $\mathscr{A}$, который инвариантен под действием $\alpha$ группь $\mathbb{R}$. Пусть $\mathscr{N}$ полуконечная алгебра фон Неймана $\left(\widetilde{\pi}\left(\mathscr{A} \times_{\alpha} \mathbb{R}\right)\right)^{\prime \prime}$, и пусть $\mathscr{D}$ - инфинитезимальный генератор канонического представления $\lambda$ группы $\mathbb{R}$ в $U(\mathscr{N})$. Тогда представление $\pi: \mathscr{A} \rightarrow \mathscr{N}$ определяет $\mathscr{L}^{(1, \infty)}$-суммируемый модуль БрейераФредгольма $(\mathscr{N}, \mathscr{D})$ для $\mathscr{A}$. Более того, если $P$ - неотрицательный спектральный проектор для $\mathscr{D} u \in \in U(\mathscr{A})$ также принадлежит области определения $\delta$, то $T_{u}:=P \pi(u) P$ является оператором Брейера-Фредгольма в $P \mathscr{N} P$ $u$

$$
\operatorname{ind}\left(T_{u}\right)=\frac{1}{2 \pi i} \tau\left(u \delta\left(u^{*}\right)\right)
$$

ДокАзАтельство. Легко удостовериться, что $\mathscr{D}$ удовлетворяет условию $\left(1+\mathscr{D}^{2}\right)^{-1 / 2} \in \mathscr{L}^{(1, \infty)}$. Согласно приведенным выше рассуждениям, для любого $a \in \operatorname{dom}(\delta)$ мы имеем $\pi(\delta(a))=2 \pi i[\mathscr{D}, \pi(a)]$. Поскольку область определения $\delta$ плотна в $\mathscr{A}$, мы видим, что $\pi$ определяет $\mathscr{L}^{(1, \infty)}$-суммируемый модуль Брейера-Фредгольма для $\mathscr{A}$.

По теореме 13.2

$$
\operatorname{ind}\left(T_{u}\right)=\lim _{p \rightarrow 1^{+}} \frac{1}{2}(p-1) \operatorname{Tr}\left(\pi(u)\left[\mathscr{D}, \pi\left(u^{*}\right)\right]\left(1+\mathscr{D}^{2}\right)^{-p / 2}\right),
$$


и, следовательно,

$$
\begin{aligned}
\operatorname{ind}\left(T_{u}\right) & =\lim _{p \rightarrow 1^{+}} \frac{1}{2}(p-1) \frac{1}{2 \pi i} \operatorname{Tr}\left(\pi\left(u \delta\left(u^{*}\right)\right)\left(1+\mathscr{D}^{2}\right)^{-p / 2}\right) \\
& =\lim _{p \rightarrow 1^{+}} \frac{1}{2}(p-1) \frac{1}{2 \pi i} \tau\left(u \delta\left(u^{*}\right)\right) \int_{-\infty}^{\infty}\left(1+t^{2}\right)^{-p / 2} d t \\
& =\lim _{p \rightarrow 1^{+}} \frac{1}{2 \pi i} \tau\left(u \delta\left(u^{*}\right)\right) \frac{1}{2}(p-1) C_{p / 2}=\frac{1}{2 \pi i} \tau\left(u \delta\left(u^{*}\right)\right) .
\end{aligned}
$$

\section{7. Класс Хохшильда характера Черна}

Теорема, которую мы обсуждаем в этом разделе, в случае $\mathscr{N}=\mathscr{L}(\mathscr{H})$ и $1<p<\infty$ ( $p$ - целое) была доказана в лекциях А. Конна в Коллеж де Франс в 1990 г. Версия этого рассуждения появилась в книге [8]. Обобщение этого рассуждения на общие полуконечные алгебры фон Неймана при дополнительном предположении, заключающемся в том, что неограниченный самосопряженный оператор $\mathscr{D}$ (который составляет часть спектральной тройки) имеет ограниченный обратный, представлено М.-Т. Бенамью и Т. Факом в [22]. Более простая стратегия, использующая псевдодифференциальное исчисление Конна-Московичи [85], была сообщена нам Найджелом Хигсоном. В соединении с результатами работы [29] рассуждения Хигсона, по-видимому, обобщаются на полуконечный случай, однако мы не будем описывать здесь детали, сосредоточившись вместо этого на другом подходе.

В работе [25] теоремы Конна и Бенамью-Фака были обобщены. Во-первых, дано доказательство в случае $p=1$, и, во-вторых, в случае типа $I I_{\infty}$ устранено предположение о том, что $\mathscr{D}$ имеет ограниченный обратный. Это важно ввиду явления "нуль в спектре" для оператора $\mathscr{D}$. Именно, для алгебры $\mathscr{N}$ типа $I I$ нуль, вообще говоря, принадлежит точечному и/или непрерывному спектру [94], и, следовательно, мы не имеем дело только с такой простой проблемой, которая возникает в случае типа $I$, вызванной конечномерным ядром. Одна из характерных черт подхода работы [25] состоит в том, что стратегия доказательства одинакова для всех $p \geqslant 1$ и не зависит от типа алгебры фон Неймана $\mathscr{N}$.

Мы объясним общую полуконечную версию результата, полученного в случае типа $I$ в $[7 ;$ IV.2. $\gamma]$, который вычисляет класс Хохшильда характера Черна $(p, \infty)$-суммируемой спектральной тройки.

Теорема 17.1. Пусть $(\mathscr{A}, \mathscr{H}, \mathscr{D})$ - $(p, \infty)$-суммируемая спектральная тройка класса $Q C^{k}$ с цельм $p \geqslant 1$ u $k=\max \{2, p-2\}$. Тогда

1) кочикл Хохшилъда на $\mathscr{A}$ определяется формулой

$$
\varphi_{\omega}\left(a_{0}, \ldots, a_{p}\right)=\lambda_{p} \tau_{\omega}\left(\Gamma a_{0}\left[\mathscr{D}, a_{1}\right] \cdots\left[\mathscr{D}, a_{p}\right]\left(1+\mathscr{D}^{2}\right)^{-p / 2}\right)
$$

2) для всех р-ииклов Хохиильда $c \in C_{p}(\mathscr{A})($ m.e. $b c=0)$

$$
\left\langle\varphi_{\omega}, c\right\rangle=\left\langle\mathrm{Ch}_{F_{\mathscr{D}}}, c\right\rangle
$$

где $\mathrm{Ch}_{F_{\mathscr{D}}}$ - характер Черна в ииклических когомологиях фредгольмова предмодуля над $\mathscr{A}$ с $F_{\mathscr{D}}=\mathscr{D}\left(1+\mathscr{D}^{2}\right)^{-1 / 2}$. 
ЗАмечАниЕ. Здесь $\tau_{\omega}$ обозначает след Диксмье, ассоциированный с любым состоянием $\omega \in D\left(\mathbb{R}_{+}^{*}\right)$. Двумя самыми важными следствиями из теоремы 17.1 являются следующие.

СлеДСтвиЕ 17.2. Пусть тройка $(\mathscr{A}, \mathscr{H}, \mathscr{D})$ такова, как в теореме 17.1. Если с $=\sum_{i} a_{0}^{i} \otimes a_{1}^{i} \otimes \cdots \otimes a_{p}^{i}-p$-цикл Хохиильда, то оператор

$$
\Gamma \sum_{i} a_{0}^{i}\left[\mathscr{D}, a_{1}^{i}\right] \cdots\left[\mathscr{D}, a_{p}^{i}\right]\left(1+\mathscr{D}^{2}\right)^{-p / 2}
$$

измерим (по Диксмье).

Это следствие имеет отношение к аксиомам некоммутативной спинорной геометрии, поскольку оно говорит о том, что если мы используем теорию некоммутативного интегрирования, задаваемую следом Диксмье, нам не надо беспокоиться о том, какой функционал $\omega$ мы используем, чтобы определить интегрирование, когда мы применяем его к циклам Хохшильда.

СлЕДСТвИЕ 17.3. Пусть $(\mathscr{A}, \mathscr{H}, \mathscr{D})$ как в теореме $17.1 ;$ предположим, что $\mathrm{Ch}_{F_{\mathscr{D}}}$ имеет нетривиальное спаривание с $H_{\mathrm{p}}(\mathscr{A})$. Тогда

$$
\tau_{\omega}\left(\left(1+\mathscr{D}^{2}\right)^{-p / 2}\right) \neq 0 .
$$

ЗАмЕчАнИЕ. Условия данного следствия заключаются в том, что существует некоторый $p$-цикл Хохшильда такой, что $\left\langle I \mathrm{Ch}_{F_{\mathscr{D}}}, c\right\rangle \neq 0$, где отображение $I$ определено А. Конном [7; п. III.1. $\gamma$, с. 199-200]. Вычисляя данное спаривание при помощи приведенной выше теоремы 17.1, мы видим, что оператор $\left(1+\mathscr{D}^{2}\right)^{-p / 2}$ не может иметь нулевой след Диксмье при любом выборе функционала Диксмье $\omega$. Действительно, если бы след Диксмье оператора $\left(1+\mathscr{D}^{2}\right)^{-p / 2}$ равнялся нулю, то для любого цикла Хохшильда $c=\sum_{i} a_{0}^{i} \otimes \cdots \otimes a_{p}^{i}$ мы имели бы

$$
\begin{aligned}
\left|\left\langle I \mathrm{Ch}_{F_{\mathscr{D}}}, c\right\rangle\right| & =\left|\sum_{i} \tau_{\omega}\left(\Gamma a_{0}^{i}\left[\mathscr{D}, a_{1}^{i}\right] \cdots\left[\mathscr{D}, a_{p}^{i}\right]\left(1+\mathscr{D}^{2}\right)^{-p / 2}\right)\right| \\
& \leqslant \sum_{i}\left\|\Gamma a_{0}^{i}\left[\mathscr{D}, a_{1}^{i}\right] \cdots\left[\mathscr{D}, a_{p}^{i}\right]\right\| \tau_{\omega}\left(\left(1+\mathscr{D}^{2}\right)^{-p / 2}\right)=0,
\end{aligned}
$$

что невозможно для нетривиального спаривания. Следовательно, если спаривание нетривиально, след Диксмье не может обращаться в нуль на $\left(1+\mathscr{D}^{2}\right)^{-p / 2}$.

Доказательство теоремы 17.1 содержит тонкие места. Сначала предполагается, что тройка $(\mathscr{A}, \mathscr{H}, \mathscr{D})$ имеет обратимый оператор $\mathscr{D}$ (с заменой, если необходимо, $(\mathscr{A}, \mathscr{H}, \mathscr{D})$ на $\left(\mathscr{A}, \mathscr{H}^{2}, \mathscr{D}_{m}\right)$ с $\left.\mathscr{D}_{m}=\left(\begin{array}{cc}\mathscr{D} & m \\ m & -\mathscr{D}\end{array}\right)\right)$. Затем надо проверить, что $\varphi_{\omega}$ в теореме не зависит от такой замены. Также требуется проверить, что функционал $\varphi_{\omega}$, на самом деле, является коциклом Хохшильда, но это достаточно просто сделать.

Лемма 17.4. Пусть $p \geqslant 1$, и путь $(\mathscr{A}, \mathscr{H}, \mathscr{D})-(p, \infty)$-суммируемая спектральная тройка класса $Q C^{1}$. Тогда полилинейный функчионал

$$
\varphi_{\omega}\left(a_{0}, \ldots, a_{p}\right)=\lambda_{p} \tau_{\omega}\left(\Gamma a_{0}\left[\mathscr{D}, a_{1}\right] \cdots\left[\mathscr{D}, a_{p}\right]\left(1+\mathscr{D}^{2}\right)^{-p / 2}\right)
$$

является кочиклом Хохшилъда. 
ДокАЗАТЕЛЬСтво. Согласно лемме 3 работы [25] и следовому свойству следа Диксмье, мы имеем

$$
\begin{aligned}
\left(b \varphi_{\omega}\right)\left(a_{0}, \ldots, a_{p}\right)=(-1)^{p-1} \lambda_{p} \tau_{\omega}\left(\Gamma a_{0}\left[\mathscr{D}, a_{1}\right] \cdots\left[\mathscr{D}, a_{p-1}\right] a_{p}\left(1+\mathscr{D}^{2}\right)^{-p / 2}\right) \\
-(-1)^{p-1} \lambda_{p} \tau_{\omega}\left(\Gamma a_{0}\left[\mathscr{D}, a_{1}\right] \cdots\left[\mathscr{D}, a_{p-1}\right]\left(1+\mathscr{D}^{2}\right)^{-p / 2} a_{p}\right) .
\end{aligned}
$$

Поскольку тройка $(\mathscr{A}, \mathscr{H}, \mathscr{D})$ принадлежит классу $Q C^{1}$,

$$
\left[\left(1+\mathscr{D}^{2}\right)^{-p / 2}, a_{p}\right]=-\sum_{k=0}^{p-1}\left(1+\mathscr{D}^{2}\right)^{-(p-k) / 2}\left[\left(1+\mathscr{D}^{2}\right)^{1 / 2}, a_{p}\right]\left(1+\mathscr{D}^{2}\right)^{-(1+k) / 2}
$$

и этот оператор является ядерным. Таким образом, справедливо равенство $a_{p}\left(1+\mathscr{D}^{2}\right)^{-p / 2}=\left(1+\mathscr{D}^{2}\right)^{-p / 2} a_{p}$ по модулю ядерных операторов, и, тем самым, два приведенных выше члена сокращаются.

\section{8. Формула типа Лидского для следов Диксмье}

Полуконечный аналог классической теоремы Лидского, формулируемый в терминах (так называемой) спектральной меры Брауна $\mu_{T}$ оператора $T \in \mathscr{N}$, утверждает [95], что

$$
\tau(T)=\int_{\sigma(T) \backslash\{0\}} \lambda d \mu_{T}(\lambda) .
$$

В случае, когда $\mathscr{N}=\mathscr{L}(\mathscr{H})$ и $\tau$ - стандартный след $\mathrm{Tr}$, приведенное выше равенство сводится к классическому случаю, утверждающему, что след $\operatorname{Tr}(T)$ произвольного ядерного оператора $T$ задается суммой $\sum_{n \geqslant 1} \lambda_{n}(T)$, где $\left\{\lambda_{n}(T)\right\}_{n \geqslant 1}$ - последовательность собственных значений оператора $T$, выстроенная в убывающем порядке абсолютных значений чисел $\lambda_{n}$ с учетом кратностей. Заметим, что в случае $T \geqslant 0$ равенство $\operatorname{Tr}(T)=\sum_{n \geqslant 1} \lambda_{n}(T)$ немедленно следует из спектральной теоремы для компактных операторов. Если $T^{*}=T$, тогда опять по спектральной теореме можно выбрать ортонормированный базис в $\mathscr{H}$, состоящий из собственных значений оператора $T$, и все еще вывести теорему Лидского без особых проблем. Здесь следует заметить, что из предположения, что $T^{*}=T$ принадлежит идеалу $\mathscr{L}^{1}(\mathscr{H})$ всех ядерных операторов, вытекает абсолютная сходимость ряда $\sum_{n \geqslant 1}\left|\lambda_{n}(T)\right|$. Последний факт гарантирует сходимость ряда $\sum_{n \geqslant 1} \lambda_{n}(T)$ независимо от выбора упорядочения множества всех собственных значений оператора $T$ (в частности, для упорядочения собственных значений оператора $T$ в порядке убывания модулей).

Главное отличие этой ситуации от случая следов Диксмье в том, что ряд $\sum_{n \geqslant 1}\left|\lambda_{n}(T)\right|$ расходится для любого нормального оператора $T \in \mathscr{L}^{(1, \infty)}(\mathscr{H}) \backslash$ $\mathscr{L}^{1}(\mathscr{H})$. Поэтому, хотя мы и можем определить $\tau_{\omega}(T)$ для данного оператора $T=T^{*} \in \mathscr{L}^{(1, \infty)}(\mathscr{H})$ как разность $\tau_{\omega}\left(T_{+}\right)-\tau_{\omega}\left(T_{-}\right)$, где каждое число вычисляется по определению

$$
\underset{N \rightarrow \infty}{\omega} \frac{1}{\log (1+N)} \sum_{n=1}^{N} \lambda_{n}\left(T_{ \pm}\right)
$$


совсем непонятно, выполняется ли у нас равенство

$$
\tau_{\omega}(T)=\underset{N \rightarrow \infty}{\omega} \lim \frac{1}{\log (1+N)} \sum_{n=1}^{N} \lambda_{n}(T)
$$

для специальной нумерации множества $\left\{\lambda_{n}(T)\right\}_{n \geqslant 1}$, задаваемой убывающим порядком модулей чисел $\left|\lambda_{n}(T)\right|$, или же для любой нумерации этого множества. Эта проблема становится еще более выраженной в случае общей полуконечной алгебры фон Неймана.

Заметим, что из ограничения $\mu_{t}(T) \leqslant C / t, t \geqslant 1$, наложенного на оператор $T \in \mathscr{L}^{(1, \infty)}(\mathscr{N}, \tau)$ в приведенной ниже теореме, вытекает, что $T$ принадлежит идеалу $N\left(\psi_{1}\right)(\mathscr{N}, \tau)$ (см. определение $\psi_{1}$ в п. 2.1 и определение перестановочно-инвариантного идеала $N(\psi)$ в п. 2.2). Всюду ниже мы предполагаем, что $\omega \in C D\left(\mathbb{R}_{+}^{*}\right)$ (см. п. 5.2). Когда мы применяем $\omega$ к последовательности $x \in \ell_{\infty}$, мы отождествляем $x$ с его образом $i(x)$ в $L_{\infty}\left(\mathbb{R}_{+}\right)$(см. замечание 5.3).

ТЕОРема 18.1 [21]. (i) Если $(\mathscr{N}, \tau)$ - полуконечная алгебра фон Неймана $и$ $T \in \mathscr{L}^{(1, \infty)}(\mathscr{N}, \tau)$ удовлетворяет условию $\mu_{t}(T) \leqslant C / t, t \geqslant 1$, при некотором $C>0, m o$

$$
\tau_{\omega}(T)=\underset{t \rightarrow \infty}{\omega} \lim \frac{1}{\log (1+t)} \int_{\lambda \notin \frac{1}{t} G} \lambda d \mu_{T}(\lambda) .
$$

(ii) Пусть $T$ - такой компактный оператор в гильбертовом пространстве $\mathscr{H}$, что $\mu_{n}(T) \leqslant C / n, n \geqslant 1$, при некотором $C>0$. Пусть $\lambda_{1}, \lambda_{2}, \ldots$ список собственных значений оператора $T$ с учетом кратностей такой, что $\left|\lambda_{1}\right| \geqslant\left|\lambda_{2}\right| \geqslant \cdots$. Тогда

$$
\tau_{\omega}(T)=\lim _{t \rightarrow \infty} \frac{1}{\log (1+t)} \sum_{\lambda \in \sigma(T), \lambda \notin \frac{1}{t} G} \lambda \mu_{T}(\lambda)=\omega_{N \rightarrow \infty}-\lim \frac{1}{\log (1+N)} \sum_{i=1}^{N} \lambda_{i}
$$

где $\mu_{T}(\lambda)$ - алгебрачческая кратность собственного значения $\lambda$ u $G$ - произвольная ограниченная окрестность нуля в $\mathbb{C}$.

Здесь мы особо выделим тот факт, что $G$ - произвольная ограниченная окрестность 0 . В случае $\mathscr{N}=\mathscr{L}(\mathscr{H})$ формула $(25)$ говорит, что необходимо вычислить сумму всех собственных значений $\lambda_{j}(T)$, которые не принадлежат "сжатой" окрестности $\frac{1}{t} G$ при $t \rightarrow \infty$, и затем применить $\omega$-предел. Интересно отметить специальный случай формулы (25) для измеримых операторов, принадлежащих идеалу $N\left(\psi_{1}\right)$. Следует сравнивать приведенный ниже результат с результатами теорем 6.6 и 6.7 .

СлеДСтвИЕ 18.2. ЕслиТ - измеримый (по Конну-Диксмъе) оператор, удовлетворяющий предположениям теоремы 18.1 (i) (соответственно (ii)), то

$$
\tau_{\omega}(T)=\lim _{t \rightarrow \infty} \frac{1}{\log (1+t)} \int_{\lambda \notin \frac{1}{t} G} \lambda d \mu_{T}(\lambda) \quad\left(\text { cooms. } \quad \lim _{N \rightarrow \infty} \frac{1}{\log (1+N)} \sum_{i=1}^{N} \lambda_{i}\right)
$$

для любого $\omega \in C D\left(\mathbb{R}_{+}^{*}\right)$. 
Зафиксируем ортонормированный базис в $\mathscr{H}$ и отождествим каждый элемент $x \in \mathscr{L}(\mathscr{H})$ с его матрицей $\left(x_{i j}\right)_{i, j=1}^{\infty}$. Хорошо известно [49], что оператор треугольного усечения $\mathfrak{T}$, задаваемый формулой

$$
\mathfrak{T}(x)= \begin{cases}x_{i j}, & i \geqslant j, \\ 0, & i<j,\end{cases}
$$

действует ограниченно из класса ядерных операторов $\mathscr{L}^{1}(\mathscr{H})$ в $\mathscr{L}^{(1, \infty)}(\mathscr{H})$. Замечая, что $\mathfrak{T}(x)-\operatorname{diag}(x)$ квазинильпотентен для каждого $x \in \mathscr{L}^{1}(\mathscr{H})$, мы получим следующее утверждение.

СлеДСтвиЕ 18.3. Оператор $\mathfrak{T}(x)$ измерим по Конну-Диксмъе для любого $x \in \mathscr{L}^{1}(\mathscr{H})$, более того, $\tau_{\omega}(\mathfrak{T}(x))=0, \omega \in C D\left(\mathbb{R}_{+}^{*}\right)$.

Мы закончим следующим результатом, немедленно вытекающим из теоремы 18.1 (см. также [57; предложение 1]).

СлеДСтвИЕ 18.4. Пусть $M$ - компактное $n$-мерное риманово многообразие, и пусть $T$ - псевдодифференциалъный оператор порядка -n на $M$. Тогда

$$
\tau_{\omega}(T)=\lim _{N \rightarrow \infty} \frac{1}{\log N} \sum_{k=1}^{N} \lambda_{k} .
$$

\section{Список литературы}

[1] J. Dixmier, "Existence de traces non normales", C. R. Acad. Sci. Paris Sér. A, 262 (1966), 1107-1108.

[2] A. Connes, "The action functional in non-commutative geometry", Comm. Math. Phys., 117:4 (1988), 673-683.

[3] M. Wodzicki, "Noncommutative residue. I: Fundamentals", K-theory, arithmetic and geometry (Moscow, 1984-1986), Lecture Notes in Math., 1289, Springer-Verlag, Berlin, 1987, 320-399.

[4] M. Adler, "On a trace functional for formal pseudo-differential operators and the symplectic structure of the Korteweg-de Vries type equations", Invent. Math., 50:3 (1978), 219-248.

[5] Ю.И. Манин, "Алгебраические аспекты нелинейных дифференциальных уравнений”, Итоги науки и техники. Соврем. проблемы матем., 11, 1978, 5-152.

[6] V. Guillemin, "A new proof of Weyl's formula on the asymptotic distribution of eigenvalues", Adv. Math., 55:2 (1985), 131-160.

[7] A. Connes, Noncommutative geometry, Academic Press, San Diego, CA, 1994.

[8] J. M. Gracia-Bondía, J. C. Várilly, H. Figueroa, Elements of noncommutative geometry, Birkhäuser Advanced Texts: Basler Lehrbücher, Birkhäuser, Boston, MA, 2001.

[9] A. Connes, "Essay on physics and noncommutative geometry", The interface of mathematics and particle physics (Oxford, 1988), Inst. Math. Appl. Conf. Ser. New Ser., 24, Oxford Univ. Press, New York, 1990, 9-48.

[10] A. Connes, J. Lott, "Particle models and noncommutative geometry", Recent advances in field theory (Annecy-le-Vieux, 1990), Nuclear Phys. B Proc. Suppl., 18 (1991), 29-47.

[11] A. Connes, "Noncommutative geometry and reality", J. Math. Phys., 36:11 (1995), 6194-6231. 
[12] W. Kalau, "Hamilton formalism in non-commutative geometry", J. Geom. Phys., 18:4 (1996), 349-380.

[13] D. Kastler, "Noncommutative geometry and basic physics", Geometry and quantum physics (Schladming, 1999), Lecture Notes in Phys., 543, Springer, Berlin, 2000, 131230.

[14] D. Kastler, "Noncommutative geometry and fundamental physical interaction: The Lagrangian level - historical sketch and description of the present situation", J. Math. Phys., 41:6 (2000), 3867-3891.

[15] A. Connes, "Trace de Dixmier, modules de Fredholm et géométrie riemannienne", Conformal field theories and related topics (Annecy-le-Vieux, 1988), Nuclear Phys. B Proc. Suppl., 5:2 (1988), 65-70.

[16] A. Connes, "Gravity coupled with matter and the foundation of non-commutative geometry", Comm. Math. Phys., 182:1 (1996), 155-176.

[17] M.F. Atiyah, "Elliptic operators, discrete groups and von Neumann algebras", Colloque "Analyse et Topologie" en l'honneur de Henri Cartan (Orsay, 1974), Astérisque, 32-33, Soc. Math. France, Paris, 1976, 43-72.

[18] S. Albeverio, D. Guido, A. Ponosov, S. Scarlatti, "Singular traces and compact operators", J. Funct. Anal., 137:2 (1996), 281-302.

[19] S. Albeverio, D. Guido, A. Ponosov, S. Scarlatti, "Singular traces and nonstandard analysis", Advances in analysis, probability and mathematical physics (Blaubeuren, 1992), Math. Appl., 314, Kluwer, Dordrecht, 1995, 3-19.

[20] S. Albeverio, D. Guido, A. Ponosov, S. Scarlatti, "Nonstandard representation of nonnormal traces", Dynamics of complex and irregular systems (Bielefeld, 1991), Bielefeld Encount. Math. Phys., VIII, World Scientific, River Edge, NJ, 1993, 1-11.

[21] N. A. Azamov, F. A. Sukochev, "A Lidskii type formula for Dixmier traces", C. $R$. Math. Acad. Sci. Paris, 340:2 (2005), 107-112.

[22] M.-T. Benameur, T. Fack, "Type II non-commutative geometry. I: Dixmier trace in von Neumann algebras", Adv. Math., 199:1 (2006), 29-87.

[23] A. L. Carey, J. Phillips, "Unbounded Fredholm modules and spectral flow", Canad. J. Math., 50:4 (1998), 673-718.

[24] A. L. Carey, J. Phillips, "Spectral flow in Fredholm modules, eta invariants and the JLO cocycle", K-Theory, 31:2 (2004), 135-194.

[25] A. L. Carey, J. Phillips, A. Rennie, F. A. Sukochev, "The Hochschild class of the Chern character for semifinite spectral triples", J. Funct. Anal., 213:1 (2004), 111-153.

[26] A. L. Carey, J. Phillips, A. Rennie, F. A. Sukochev, "The local index formula in semifinite von Neumann algebras. I: Spectral flow", Adv. Math., 202:2 (2006), 451-516.

[27] A. L. Carey, J. Phillips, A. Rennie, F. Sukochev, "The local index formula in semifinite von Neumann algebras. II: The even case", Adv. Math., 202:2 (2006), 517-554.

[28] A. L. Carey, J. Phillips, F. A. Sukochev, "On unbounded $p$-summable Fredholm modules", Adv. Math., 151:2 (2000), 140-163.

[29] A. L. Carey, J. Phillips, F. A. Sukochev, "Spectral flow and Dixmier traces", Adv. Math., 173:1 (2003), 68-113.

[30] A. Connes, "Geometry from the spectral point of view", Lett. Math. Phys., 34:3 (1995), 203-238.

[31] P. G. Dodds, B. de Pagter, E. M. Semenov, F. A. Sukochev, "Symmetric functionals and singular traces", Positivity, 2:1 (1998), 47-75.

[32] K. Dykema, T. Figiel, G. Weiss, M. Wodzicki, "Commutator structure of operator ideals", Adv. Math., 185:1 (2004), 1-79.

[33] D. Guido, T. Isola, "Singular traces for semifinite von Neumann algebras", J. Funct. Anal., 134:2 (1995), 451-485.

[34] D. Guido, T. Isola, "On the domain of singular traces", Internat. J. Math., 13:6 (2002), 667-674. 
[35] N. Kalton, F. Sukochev, "Rearrangement invariant functionals with applications to traces on symmetrically normed ideals", Canad. Math. Bull. (to appear).

[36] R. Prinzis, Traces residuelles et asymptotique du spectre d'operateurs pseudodifferentiels, Thèse, Université de Lyon, 1994.

[37] D. Guido, T. Isola, "Dimensions and singular traces for spectral triples, with applications to fractals", J. Funct. Anal., 203:2 (2003), 362-400.

[38] D. Guido, T. Isola, "Singular traces and their applications to geometry", Operator algebras and quantum field theory (Rome, 1996), Internat. Press, Cambridge, MA, 1997, 440-456.

[39] F. A. Sukochev, "Operator estimates for Fredholm modules", Canad. J. Math., 52:4 (2000), 849-896.

[40] П.Г. Доддс, Б. де Пагтер, А.А. Седаев, Е. М. Семенов, Ф.А. Сукочев, "Сингулярные симметричные функционалы", Исследования по линейным операторам и теории функций, Зап. научн. сем. ПОМИ, 290 (2002), 42-71; http://www.pdmi.ras.ru/znsl/2002/v290.html.

[41] П. Г. Доддс, Б. Пагтер, А. А. Седаев, Е. М. Семенов, Ф. А. Сукочев, “Сингулярные симметричные функционалы и банаховы пределы с дополнительными свойствами инвариантности", Изв. РАН. Сер. матем., 67:6 (2003), 111-136.

[42] С. Г. Крейн, Ю.И. Петунин, Е. М. Семенов, Интерполяиия линейных операторов, Наука, М., 1978.

[43] J. Lindenstrauss, L. Tzafriri, Classical Banach spaces. I: Sequence spaces, Ergeb. Math. Grenzgeb., 92, Springer-Verlag, Berlin, 1977.

[44] J. Lindenstrauss, L. Tzafriri, Classical Banach spaces. II: Function spaces, Ergeb. Math. Grenzgeb., 97, Springer-Verlag, Berlin, 1979.

[45] М.Ш. Браверман, А. А. Меклер, “Свойство Харди-Литлвуда для симметричных пространств", Сиб. матем. журн., 18:3 (1977), 522-540.

[46] У. Рудин, Основы математического анализа, Мир, М., 1976.

[47] T. Fack, H. Kosaki, "Generalized s-numbers of $\tau$-measurable operators", Pacific J. Math., 123:2 (1986), 269-300.

[48] T. Fack, "Sur la notion de valeur caractéristique", J. Operator Theory, 7:2 (1982), 307-333.

[49] И.Ц. Гохберг, М.Г. Крейн, Введение в теорию линейных несамосопряженных операторов в гилъбертовом пространстве, Наука, М., 1965.

[50] Ф.А. Сукочев, В.И. Чилин, "Симметричные пространства на полуконечных алгебрах фон Неймана", Докл. АН СССР, 313:4 (1990), 811-815.

[51] V.I. Chilin, F. A. Sukochev, "Weak convergence in non-commutative symmetric spaces", J. Operator Theory, 31:1 (1994), 35-65.

[52] P. G. Dodds, T. K. Dodds, B. de Pagter, "Fully symmetric operator spaces", Integral Equations Operator Theory, 15:6 (1992), 942-972.

[53] P. G. Dodds, T.K.-Y. Dodds, B. de Pagter, "Non-commutative Banach function spaces", Math. Z., 201:4 (1989), 583-597.

[54] N. Kalton, "Spectral characterization of sums of commutators, I", J. Reine Angew. Math., 504 (1998), 115-125.

[55] K. J. Dykema, N. J. Kalton, "Spectral characterization of sums of commutators, II", J. Reine Angew. Math., 504 (1998), 127-137.

[56] K. J. Dykema, N. J. Kalton, "Sums of commutators in ideals and modules of type II factors", Ann. Inst. Fourier (Grenoble), 55:3 (2005), 931-971.

[57] T. Fack, "Sums of commutators in non-commutative Banach function spaces", J. Funct. Anal., 207:2 (2004), 358-398.

[58] G. G. Lorentz, "A contribution to the theory of divergent sequences", Acta Math., 80:1 (1960), 167-190. 
[59] М. Рид, Б. Саймон, Методы современной математической физики. Т 1: Функииональный анализ, Мир, М., 1977.

[60] S. Lord, A. Sedaev, F. Sukochev, "Dixmier traces as singular symmetric functionals and applications to measurable operators", J. Funct. Anal., 224:1 (2005), 72-106.

[61] F. Cipriani, D. Guido, S. Scarlatti, "A remark on trace properties of $K$-cycles", J. Operator Theory, 35:1 (1996), 179-189.

[62] J. V. Varga, "Traces on irregular ideals", Proc. Amer. Math. Soc., 107:3 (1989), 715723.

[63] Г. Харди, Расходящиеся ряды, ИЛ, М., 1951.

[64] L. Sucheston, "Banach limits", Amer. Math. Monthly, 74 (1967), 308-311.

[65] A. Connes, "Entire cyclic cohomology of Banach algebras and characters of $\theta$ summable Fredholm modules", K-Theory, 1:6 (1988), 519-548.

[66] A. Connes, "Compact metric spaces, Fredholm modules, and hyperfiniteness", Ergodic Theory Dynam. Systems, 9:2 (1989), 207-220.

[67] A. Connes, "Non-commutative differential geometry", Inst. Hautes Études Sci. Publ. Math., 62 (1985), 41-144.

[68] M. F. Atiyah, V.K. Patodi, I. M. Singer, "Spectral asymmetry and Riemannian geometry, I", Math. Proc. Cambridge Philos. Soc., 77 (1975), 43-69.

[69] M. F. Atiyah, V.K. Patodi, I. M. Singer, "Spectral asymmetry and Riemannian geometry, III", Math. Proc. Cambridge Philos. Soc., 79:1 (1976), 71-99.

[70] E. Schrohe, M. Walze, J.-M. Warzecha, "Construction de triplets spectraux à partir de modules de Fredholm", C. R. Acad. Sci. Paris Sér. I Math., 326:10 (1998), 11951199.

[71] A. Rennie, "Smoothness and locality for nonunital spectral triples", K-Theory, 28:2 (2003), 127-165.

[72] J. Phillips, "Self-adjoint Fredholm operators and spectral flow", Canad. Math. Bull., 39:4 (1996), 460-467.

[73] J. Phillips, "Spectral flow in type I and type II factors - a new approach", Cyclic cohomology and noncommutative geometry (Waterloo, ON, 1995), Fields Inst. Commun., 17, Amer. Math. Soc., Providence, RI, 1997, 137-153.

[74] J. Phillips, I. Raeburn, "An index theorem for Toeplitz operators with noncommutative symbol space", J. Funct. Anal., 120:2 (1994), 239-263.

[75] M. Breuer, "Fredholm theories in von Neumann algebras, I", Math. Ann., 178 (1968), 243-254.

[76] M. Breuer, "Fredholm theories in von Neumann algebras, II", Math. Ann., 180:4 (1969), 313-325.

[77] V.S. Perera, Real-valued spectral flow in type $I I_{\infty}$ factor, Ph.D. Thesis, IUPUI, Indianapolis, 1993.

[78] V.S. Perera, "Real-valued spectral flow", Multivariable operator theory (Seattle, WA, 1993), Contemp. Math., 185, Amer. Math. Soc., Providence, RI, 1995, 307-318.

[79] M. T. Benameur, A. L. Carey, J. Phillips, A. Rennie, F. A. Sukochev, K. P. Wojciechowski, "An analytic approach to spectral flow in von Neumann algebras", Analysis, geometry and topology of elliptic operators, Papers in honor of Krzysztof P. Wojciechowski, Proceedings of a workshop (Roskilde, 2005), eds. B. Booß-Bavnbek et al., World Scientific, Singapore, 2006, 297-352.

[80] B. Booß-Bavnbek, M. Lesch, J. Phillips, "Unbounded Fredholm operators and spectral Flow", Canad. J. Math., 57:2 (2005), 225-250.

[81] V. Mathai, "Spectral flow, eta invariants, and von Neumann algebras", J. Funct. Anal., 109:2 (1992), 442-456.

[82] V.S. Perera, "Real valued spectral flow in a type $\mathrm{II}_{\infty}$ factor", Houston J. Math., 25:1 (1999), 55-66. 
[83] E. Getzler, "The odd Chern character in cyclic homology and spectral flow", Topology, 32:3 (1993), 489-507.

[84] J.-L. Loday, Cyclic homology, Grundlehren Math. Wiss., 301, Springer-Verlag, Berlin, 1998.

[85] A. Connes, H. Moscovici, "The local index formula in noncommutative geometry", Geom. Funct. Anal., 5:2 (1995), 174-243.

[86] У. Браттели, Д. Робинсон, Операторные алгебры и квантовая статистическая механика: $C^{*}-$ u $W^{*}$-алгебры. Группы симметрий. Разложение состояний, Мир, M., 1982.

[87] L. A. Coburn, R. G. Douglas, D. G. Schaeffer, I. M. Singer, " $C^{*}$-algebras of operators on a half-space. II: Index theory", Inst. Hautes Études Sci. Publ. Math., 40 (1971), 69-79.

[88] M. Lesch, "On the index of the infinitesimal generator of a flow", J. Operator Theory, 26:1 (1991), 73-92.

[89] N. Higson, "The local index formula in noncommutative geometry", Contemporary developments in algebraic K-theory, ICTP Lect. Notes, XV, Abdus Salam Int. Cent. Theoret. Phys., Trieste, 2004, 443-536.

[90] A. Connes, "Sur la théorie non commutative de l'intégration", Algèbres d'opérateurs (Les Plans-sur-Bex, 1978), Lecture Notes in Math., 725, Springer-Verlag, Berlin, 1979, 19-143.

[91] М. А. Шубин, "Псевдодифференциальные почти-периодические операторы и алгебры фон Неймана", Тp. MMO, 35 (1976), 103-164.

[92] М. А. Шубин, "Спектральная теория и индекс эллиптических операторов с почти периодическими коэффициентами”, УМН, 34:2 (1979), 95-135.

[93] J. Dixmier, Les algèbres d'opérateurs dans l'espace hilbertien (algèbres de von Neumann), Cahier Scientifiques, XXV, Gauthier-Villars, Paris, 1969.

[94] M. Farber, S. Weinberger, "On the zero-in-the-spectrum conjecture", Ann. of Math. (2), 154:1 (2001), 139-154.

[95] L. G. Brown, "Lidskii's theorem in the type II case", Geometric methods in operator algebras (Kyoto, 1983), Pitman Res. Notes Math. Ser., 123, Longman Sci. Tech., Harlow, 1986, 1-35.

\section{А. Л. Кери (А. L. Carey)}

Mathematical Sciences Institute,

Australian National University

E-mail: acarey@maths .anu.edu.au

Ф. А. Сукочев (F. A. Sukochev)

School of Informatics and Engineering, Flinders University

E-mail: sukochev@infoeng.flinders.edu.au
Поступила в редакцию

10.11 .2005 